\title{
Molecular Sizing using Fluorescence Correlation Spectroscopy
}

\author{
Dissertation \\ for the award of the degree \\ “Doctor rerum naturalium” (Dr.rer.nat.) \\ Division of Mathematics and Natural Sciences \\ of the Georg-August-Universität Göttingen
}

submitted by

Anastasia Loman

from Minsk, Belarus

Göttingen 2010 
Members of the Thesis Committee:

\section{Prof. Dr. J. Enderlein (Reviewer)}

Group of "Single molecule spectroscopy and imaging for Biophysics and Complex systems", Faculty of Physics, $3^{\mathrm{d}}$ Institute of Physics - Biophysics, Georg-August-University Göttingen

\section{Prof. Dr. F.S. Wouters (Reviewer)}

Laboratory for Molecular and Cellular Systems, Department of Neuro- and Sensory Physiology Centre II, Physiology and Pathophysiology

Georg-August-University Göttingen,

\section{Prof. Dr. H. Grubmüller}

Department of Theoretical and Computer-based Biophysics, Max Planck Institute for Biophysical Chemistry

Date of the oral examination: 29.06.2010 


\section{Affidavit}

Hereby, I declare that the presented thesis has been written independently and with no other sources and aids than quoted.

Gottingen, 21.04.2010 


\section{Acronyms}

$\begin{array}{ll}\text { ACF } & \text { autocorrelation function } \\ \text { BSA } & \text { bovine serum albumin } \\ \text { CCF } & \text { cross-correlation function } \\ \text { DIC } & \text { differential interference contrast } \\ \text { DLS } & \text { dynamic light scattering } \\ \text { DMSO } & \text { dimethyl sulfoxide } \\ \text { FCS } & \text { fluorescence correlation spectroscopy } \\ \text { 2fFCS } & \text { dual-focus fluorescence correlation spectroscopy } \\ \text { HPLC } & \text { high performance liquid chromatography } \\ \text { HSA } & \text { human serum albumin } \\ \text { MDF } & \text { molecule detection function } \\ \text { MHKS } & \text { Mark-Houwink-Kuhn-Sakurada } \\ \text { MW } & \text { molecular weight } \\ \text { NHS } & \text { N-hydroxysuccinimid } \\ \text { pfgNMR } & \text { pulsed field gradient nuclear magnetic resonance }\end{array}$

\section{Symbols for amino acids}

$\begin{array}{lll}\text { A } & \text { Ala } & \text { alanine } \\ \text { B } & \text { Asx } & \text { asparagine or aspartic acid } \\ \text { C } & \text { Cys } & \text { cysteine } \\ \text { D } & \text { Asp } & \text { aspartic acid } \\ \text { E } & \text { Glu } & \text { glutamic acid } \\ \text { F } & \text { Phe } & \text { phenylalanine } \\ \text { G } & \text { Gly } & \text { glycine } \\ \text { H } & \text { His } & \text { histidine } \\ \text { I } & \text { Ile } & \text { isoleucine } \\ \text { K } & \text { Lys } & \text { lysine } \\ \text { L } & \text { Leu } & \text { leucine } \\ \text { M } & \text { Met } & \text { methionine } \\ \text { N } & \text { Asn } & \text { asparagine } \\ \text { P } & \text { Pro } & \text { proline } \\ \text { Q } & \text { Gln } & \text { glutamine } \\ \text { R } & \text { Arg } & \text { arginine } \\ \text { S } & \text { Ser } & \text { serine } \\ \text { T } & \text { Thr } & \text { threonine } \\ \text { V } & \text { Val } & \text { valine } \\ \text { W } & \text { Trp } & \text { tryptophan } \\ \text { Y } & \text { Tyr } & \text { tyrosine } \\ \text { Z } & \text { Glx } & \text { glutamine or glutamic acid }\end{array}$




\section{Content}

1 Introduction...............................................................................................

1.1 Historical background...........................................................................2

1.2 Molecular sizing techniques ....................................................................

1.3 Fluorescence Correlation Spectroscopy ……………...............................

1.4 Dual-focus Fluorescence Correlation Spectroscopy ……………….........11

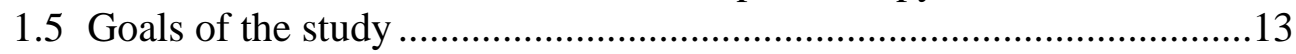

2 Experimental set-up and methods............................................................15

2.1 Dual-focus Fluorescence Correlation Spectroscopy. Optical set-up .....15

2.2 Calculating the auto- and cross-correlation functions .............................17

2.3 Calibration of the shear distance of the Nomarski-prism .........................19

2.4 Absolute and precise diffusion coefficient measurements across the visible spectrum ..................................................................................23

3 Performance of dual-focus Fluorescence Correlation Spectroscopy under optical saturation. .................................................................................22

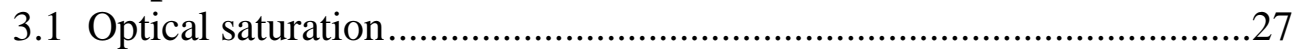

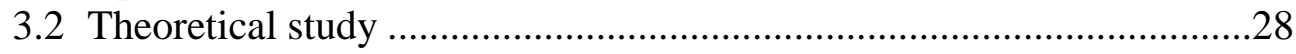

3.3 Cy5 diffusion under optical saturation .................................................32

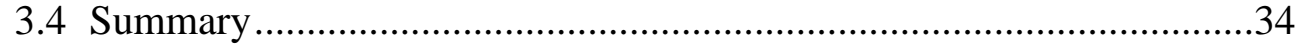

4 Molecular sizing with dual-focus fluorescence correlation spectroscopy..................................................................................................37

4.1 Accuracy of dual-focus fluorescence correlation spectroscopy ..............37

4.2 Quantitative relation between molecular weight

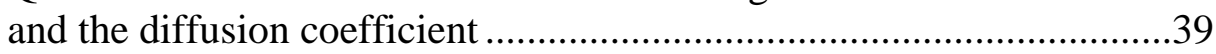

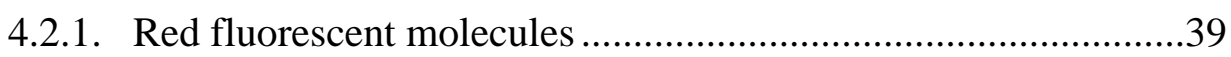

4.2.2. Short peptides.................................................................................40

4.2.3. Discussion ...............................................................................42

4.3 Globular protein sizing .........................................................................4

4.4 Materials and methods .........................................................................52

5 Rotational diffusion and protein sizing.......................................................53

5.1 Theory ......................................................................................54

5.1.1. Autocorrelation function ............................................................54

5.1.2. Excitation and detection...........................................................56

5.1.3. Molecular shape and rotational diffusion ...................................60

5.2 Materials and methods ...........................................................................63

5.2.1. Measurement set-up .....................................................................63

5.2.2. Calculation of the ACF .............................................................63

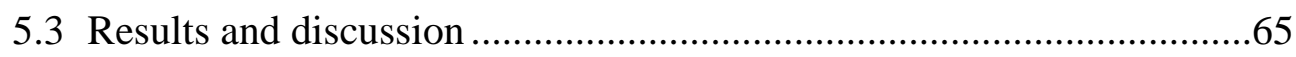

6 Summary........................................................................................................71

Appendix ...........................................................................................................75

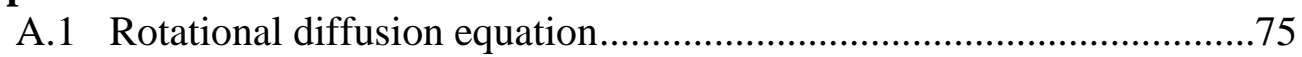

A.2 Molecular detection function ..................................................................80

A.3 Summed up results for 2fFCS and nanosecond time-scale FCS...........82

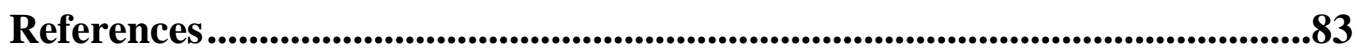



If, in some cataclysm, all of scientific knowledge were to be destroyed, and only one sentence passed on to the next generations of creatures, what statement would contain the most information in the fewest words? I believe it is the atomic hypothesis (or the atomic fact, or whatever you wish to call it) that all things are made of atoms-little particles that move around in perpetual motion, attracting each other when they are a little distance apart, but repelling upon being squeezed into one another. In that one sentence, you will see, there is an enormous amount of information about the world, if just a little imagination and thinking are applied.

Feynman "Lectures on Physics"

\section{Introduction}

Size is one of the basic characteristics of molecules. Molecular size can be a very sensitive detector of changes in environment such as temperature, $\mathrm{pH}$ or chemical composition (Lu et al., 1993; Sherman et al., 2008; Parmar \& Muschol, 2009). Molecular size also changes upon interactions with other molecules, e.g. binding of ions (Yamniuk et al., 2004). Therefore, the ability to determine molecular size and moreover to observe its changes can provide a wealth of information about molecules and their interactions (Sun, 2004; Wilson \& Walker, 2010). As such, precise measurements of molecular size find broad applications in physics, chemistry and biology (for example, Murphy \& Tsai, 2006).

The challenge of measuring the molecular size is to do it with sufficient accuracy. An accuracy in the order of Ångstrøms is necessary to distinguish, for example, between different conformational states in proteins (Weljie et al., 2003). Another challenge is to perform these measurements close to the infinite dilution limit to prevent any intermolecular interaction of aggregation that would alter the correct size value (Kiefhaber et al., 1991). Thus molecular sizing is a state-of-art research performed on a cross-road of physics, biology and chemistry.

The work presented here is concerned with high-precision sizing of molecules at pico- to nanomolar concentrations. A special spectroscopic technique of single-molecule spectroscopy, namely fluorescence correlation spectroscopy (FCS) was chosen because of its enormous sensitivity and relative simplicity, as will be seen below. Besides, FCS is very aesthetic from a physics point of view, because it employs one of the most basic properties of matter, thermal fluctuations: "Alive or not, everything is subject to thermal fluctuations" (Berg, 1983).

In what follows, I will start from a historical background, followed by an overview of the various methods that are used for sizing molecules in solution. Then, I 
will focus on the general introduction into the philosophy of FCS and, in particular, on one advanced special variation of FCS, dual-focus FCS or 2fFCS, which proved to be of fundamental importance for obtaining high-precision values of molecular size.

\subsection{Historical background}

The history of molecule is closely interwoven with the history of atom. Moreover till late into the 18 th century, the term "molecule" was used synonymously with "atom" to denote an "extremely minute particle".

The idea of atom belongs to the ancient Greek philosophers Leucippus and Democritus. They surmised that there are atoms because there must be a limit to the divisibility of matter. Then, it took almost two millennia to step from that speculative philosophical idea to a scientific theory based on careful chemical, stoichiometric measurements as formulated by the chemist John Dalton. His work was directly related to the concept of "a molecule" as being two or more atoms bound by strong chemical bonds. But only by the beginning of the 20th century, with Einstein's theoretical work and Perrin's experiments on Brownian motion, the existence of atoms and molecules was finally proven beyond any doubt.

With the acceptance of the existence of atoms and molecules and the development of quantum mechanics, within several decades many different methods were developed for the study of molecular structure. Among them were nuclear magnetic resonance (NMR), x-ray diffraction, electron microscopy, and different kinds of optical spectroscopy. Typically, these methods allowed for measuring the average characteristics of a large number of atoms or molecules (ensemble or bulk measurements). Only the second half of the 20th century saw the development of real single atom or molecule measurement techniques such as single-molecule fluorescence spectroscopy, field emission microscopy, or atomic force microscopy (AFM). These techniques opened new vistas for studying processes and properties on a single particle level which was impossible to do before. Here, the starting point of single-molecule fluorescence spectroscopy in condensed matter can be traced back to the groundbreaking work of W. E. Moerner and L. Kador in 1989 (Moerner \& Kador, 1989) and, independently, by Michel Orrit and Jacky Bernard, who performed hole-burning experiments on the fluorescence of immobilized single molecules in ultrathin films crystals at liquid helium temperatures. ( 1.5 K) (Orrit \& Bernard, 1990). However, the big break-through in detecting directly fluorescent light from an individual molecule in 
solution under ambient conditions came with the successful detection of single rhodamine molecules in water by the group of Richard Keller in 1987 (Nguyen et al., 1987). This launched an avalanche of methodological developments and innumerable applications that continues up to this day.

Nowadays, due to the development of new and cheap laser sources, high-sensitive single-photon counting detectors, and with the advent of high-quality objectives with large numerical aperture, single-molecule fluorescence spectroscopy and imaging has become a standard technique in many labs around the world (see, for example, (Selvin \& Ha, 2008)). Correspondingly broad are the many different applications of the technique in the physical, chemical and biological sciences. Zander (2002), Hinterdorfer and van Qijen (2009), Knight (2009) are only few text books giving an overview of a modern single molecule field state.

\subsection{Molecular sizing techniques}

The hydrodynamic radius is a typical parameter for characterizing molecular size. The radius is directly coupled to a diffusion coefficient of a particle via Stokes-Einstein relation. Any change in that radius will change the associated diffusion coefficient of the molecules. The diffusion coefficient is the fundamental parameter describing diffusion of a molecule in a solution. There are several standard methods to measure molecular motion (and thus size) in solutions with high accuracy. Among them are dynamic light scattering (DLS), pulsed field gradient NMR (Callaghan, 1991), analytical ultracentrifugation, and fluorescence correlation spectroscopy (FCS).

The main advantage of DLS is its ability to study untagged molecules, as this method is based solely on light scattering. The core idea is to register scattered laser light from a sample and to evaluate the time-dependent fluctuations in scattering intensity which is due to the constantly changing distances between diffusing molecules in solution. The recorded scattering signal is autocorrelated, yielding the second order or autocorrelation function (ACF). The temporal decay of the ACF contains information about the particles' diffusion coefficient and thus their hydrodynamic radius. However, due to the scaling of the scattering intensity with molecular volume, the method becomes increasingly insensitive with decreasing radius, making it rather applicable to objects larger than $\sim 10 \mathrm{~nm}$, and demanding large sample concentrations (micromolar and larger) (Berne \& Pecora, 2000).

There are two ways to study diffusion processes with NMR and to measure 
diffusion coefficients: analysis of relaxation data, and pulsed-field gradient or pfgNMR. The relaxation method is in fact sensitive to rotational diffusion, while in pgfNMR measurement, motion is measured over millisecond to seconds (W.S. Price, 1997). In pfgNMR experiment two subsequent gradient pulses are used which each produce opposite phase shift. For a sample in which the spins do not change position over the time between both pulses, the resulting phase shift is zero. However, should spin migrate, residual phase shifts arise which are sensitive to motion (Callaghan et al., 1999). The limit on measurable diffusion values, therefore, depends on the area of the gradient pulses and the time allowed for diffusion. Larger values of diffusion coefficient $\left(\geq 10^{-5} \mathrm{~cm}^{2} / \mathrm{s}\right)$ can be reliably measured using this experiment. In case of large molecules, such as proteins, difficulties in measuring diffusion occur in obtaining sufficient amplitude attenuation as well as in water suppression. Because of the importance of observing exchangeable protons, NMR solution conditions usually require a $10^{5}$-fold excess of water protons which means at 1-2 $\mathrm{mM}$ protein concentration an solvent consisting of $90 \% \mathrm{H}_{2} \mathrm{O} / 10 \% \mathrm{D}_{2} \mathrm{O}$ (Altieri et al., 1995).

An analytical ultracentrifugation uses advantages of a preparative ultracentrifuge and an optical detection system. It is capable of directly measuring the sample concentration inside the centrifuge cell during sedimentation. The sample is visualized in real time during sedimentation. There are two experimental applications: sedimentation velocity and sedimentation equilibrium. Sedimentation equilibrium is a thermodynamic technique that is sensitive to the mass but not the shape of the macromolecular species. In contrast, sedimentation velocity is a hydrodynamic technique sensitive to the mass and shape of the macromolecular species (Brown \& Schuck, 2006). In a sedimentation velocity experiment, a moving boundary is formed on application of a strong centrifugal field. A series of scans (i.e., measurements of sample concentration as a function of radial distance) are recorded at regular intervals to determine the rate of movement that contains information about molecule's mass, and broadening of the boundary as a function of time providing shape characterization. Depending on the application and optical system used, sample requirements can differ (Cole et al., 1999), hence the experiments are often carried out at micromolar concentration (Altieri et al., 1995).

All above mentioned methods have their advantages to solve different problems but all of them operate at rather high sample concentrations, far away from the limit of infinite dilution. To estimate correctly hydrodynamic radius, one has often to measure at 
different concentrations and to extrapolate the concentration/diffusion coefficient curve towards zero concentration (e.g. see Liu et al., 2005). Another problem is that proteins are often prone to aggregation at the concentrations needed for obtaining sufficient data quality (Kiefhaber et al., 1991).

In comparison with others methods FCS is designed to work at nanomolar concentration, i.e. close to infinite dilution limit. Consequently, values for the diffusion coefficient obtained by FCS are indistinguishable from their infinite dilution value, and the method circumvents most aggregation problems. Another significant advantage of FCS is its relative technical simplicity, at least when compared to such methods as NMR. How FCS achieves those advantages as well as possible difficulties one can face using this method are discussed in the next section.

\subsection{Fluorescence Correlation Spectroscopy}

FCS is a fluorescence technique where the focus of interest is shifted from average emission intensity itself to intensity fluctuations caused by the minute deviation of small system at thermal equilibrium.

The idea of FCS was introduced by Madge, Elson and Webb in 1972. They applied FCS to measure diffusion and chemical dynamic of DNA-ethidium bromide interaction (Magde et al., 1972). But only in early nineties the full potential of the method and its extreme sensitivity was demonstrated by Rigler and his coworkers owing to both new developments in laser technique, microscopy and their idea to combine FCS technique with confocal detection (Rigler et al., 1990, Woffbeis 1992, Rigler et al., 1993).

There are two important things in FCS: a nanomolar sample concentration and a very small volume where excitation as well as
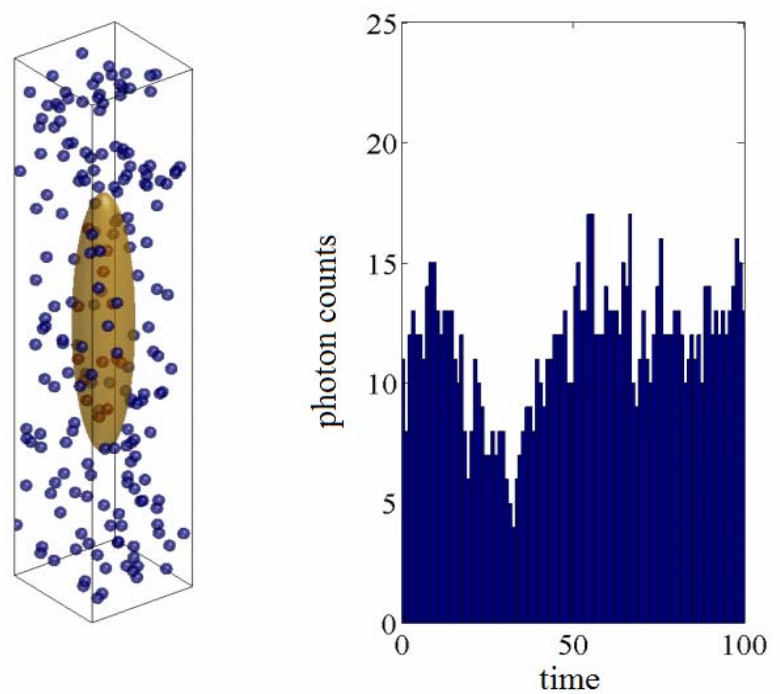

Fig.1.1: The left panel shows a schematic of the detection volume (yellow) where effective fluorescence detection takes place. When molecules (blue spheres) diffuse in and out of that region, they generate a highly fluctuating fluorescence signal (right panel) due to ever changing numbers of molecules within the detection volume. 
detection is confined. The volume restriction is essential part of FCS because even such low concentration as one nanomolar corresponds to about $10^{11}$ particles in one milliliter. However, to be able to see the fluorescence intensity fluctuations one has to excite one or very few molecules at a time (Fig.1.1). To achieve this goal the incoming laser light is strongly focused by a high numerical aperture objective to a diffraction limited spot. A confocal pinhole, introduced in the image plane, blocks all light emanating off the focal plane and this way limits the detection volume in axial direction.

The detected fluorescence intensity trace is multiplied with a time-shifted replica of itself for different values of time shift (lag time) $\tau$. The time averages of these products are reasonable estimates of the so-called autocorrelation function $g$ (secondorder correlation function, in short ACF) for the respective lag time $\tau$ :

$$
g(\tau)=\langle I(t) I(t+\tau)\rangle
$$

$I(t)$ is the fluorescence intensity at time $t$ and $I(t+\tau)$ is the intensity at time $t+\tau$, the triangular brackets denote time averaging. The physical meaning of the autocorrelation is that it is directly proportional to the probability to detect a photon at time $\tau$ if there was a photon detection event at time zero. This probability is composed of two different terms. One and the most important term contains correlated signal, i.e. the two photons are originating from one and the same molecule and are then physically correlated. This highlights the single molecule nature of FCS. The

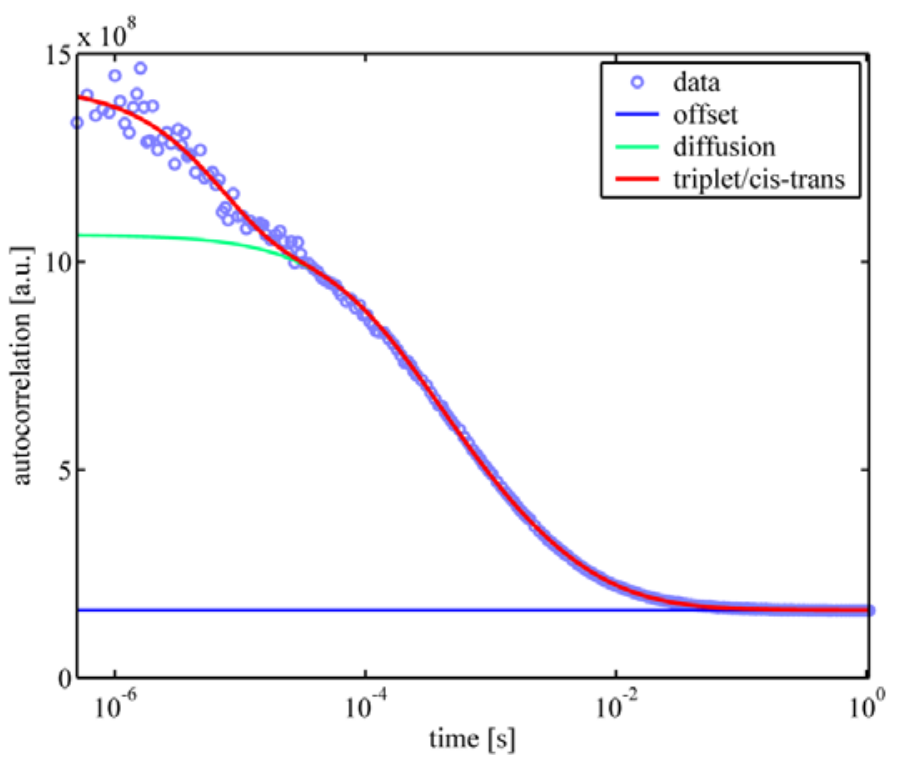

Fig.1.2: Typical autocorrelation curve: On the microsecond time scale the fast decay is due to fast photophysical processes i.e. transitions into the triplet state or cis-trans isomerisation (red). On a millisecond time scale, autocorrelation decays due to the diffusion of molecules out of the detection region (green). The long-time constant offset (blue) is due to the completely uncorrelated photon pairs. Open circles: measured data (aqueous solution of the dye Alexa633).

other term consists of all contributions from uncorrelated signals, i.e. the two detected photons were originating from different fluorescent molecules or from a backscatted 
laser light and therefore do not have any physical correlation. These uncorrelated events will contribute to a constant offset of $g(\tau)$ that is completely independent on $\tau$ (the joint probability to detect two physically uncorrelated photons is completely independent of the time distance between their detection). Typical autocorrelation curve is shown on Fig. 1.2.

From a qualitative consideration, ACF shows how long the fluorescence molecule can be observed. The closer to the center molecule stays, the more consecutive photons from the same molecule can be registered. With time the rate of detected photons decreases due to the diffusion of the molecule out of the detection region and disappears when it completely diffused away. This means the temporal

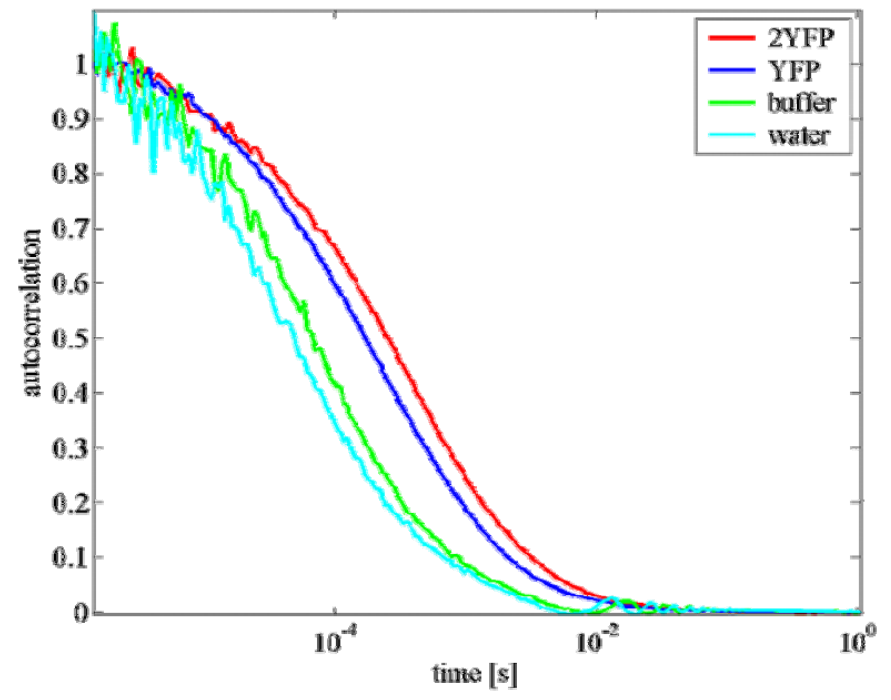

Fig.1.3: Example of the connection between autocorrelation decay and diffusion coefficient. Four measurements are shown: purified water (light blue) and a salt buffer solution (green) that still contain rapidly diffusing fluorescent contaminations; aqueous solution of the yellow fluorescent protein (YFP, blue), showing a much slower diffusion; aqueous solution of two coupled yellow fluorescent proteins bound together by a short amino linker, displaying an even slower diffusion. decay of the correlation function is proportional to the diffusion speed of the molecule and therefore related to its size. Thus, FCS measurements can provide information about diffusion of fluorescing molecules. Any process that alters the diffusion coefficient or the fluorescence of the molecule can therefore be measured by FCS. For example, consider the binding of two proteins in solution. It is well illustrated by Fig.1.3.where correlation curves of one and two coupled yellow fluorescence proteins are shown.

Another important property of the ACF is its dependence on the concentration of fluorescing molecules. It is rather obvious that the fluorescence intensity fluctuations will be larger for smaller molecule concentrations. Indeed, if one has, on average, only a signal molecule within the detection volume, than the diffusion of this molecule out of this volume or the diffusion of another molecule into this volume will cause a big change in measured fluorescence intensity. On the contrary, if the average number of 
fluorescing molecules within the detection volume is rather large (e.g. several hundreds), then the leaving or entering of a molecule causes only small signal variations. That means this method is limited to the narrow range of the sample concentrations: $10^{-13}$ $10^{-8} \mathrm{M}$. For any other concentration, the measurement time for obtaining a high-quality autocorrelation function gets prohibitively large. That may restrict FCS applications in some fields. For example, many enzymes naturally work at much higher ligand concentrations, and their Michaelis constants are often in the micro- to millimolar range (Fersht, 1999). Moreover, low concentrations of ligand can influence the mechanistic pathway of enzyme kinetics and alter the partitioning between multiple catalytic pathways, thus affecting turnover cycle histories and distributions (Levene et al., 2003; and ref. there). But there are several ways to overcome that problem: One is to rapidly scan the laser focus through the solution (Petersen, 1986; Petersen et al., 1986) and another is to reduce the observation volume that gives opportunity to work at higher concentrations. For example, Starr and coworkers combine total internal reflection and FCS to reduce a volume by an order of magnitude (Starr et al., 2001). Combination of stimulated emission and FCS brings resolution down to $90-110 \mathrm{~nm}$ in lateral dimensions and produces a focal volume 18 times smaller than one can get using conventional confocal microscopy (Klar et al., 2000, Klar et al., 2001). An advantage of employing so-called zero mode waveguide together with FCS is the flexibility to choose the best volume size and sample concentration. For detailed information about zero mode waveguide can be found in the refs. (Levene et al., 2003; Wenger et al., 2009).

To conclude, FCS is relatively simple method to implement, maintain and use what assures its wide and extensive application: Besides, the straightforward application which study molecular diffusion in free solution (see, for example, (Borsch et al., 1998; Diez et al., 2004)), FCS can be also applied to study different processes in artificial and cell membranes, where diffusion is limited to two dimensions (Benda, et al., 2003, Meissner \& Häberlein, 2003, Dertinger, et al., 2006). This technique is also used in combination with microfluidic devices where dimensions reduce from three to one due to flow in microfluidic cell (Magde, et al., 1978; Enderlein, et al., 1998; Arbour \& Enderlein, 2010). Such combination is useful to study, for example, enzymatic reactions and folding/unfolding processes (Hamadani, \& Weiss, 2008).

The new extensions such as fluorescence cross-color correlation spectroscopy, FCS together with resonance energy transfer have spread a lot in biochemical and biological fields to resolve behavior of several different molecule species or different 
parts of the same macromolecule

(Rigler \& Elson, 2001, Schwille \& Haustein 2001, Widengren et al., 2001, Eggeling et al., 2005). Another approach to extract the FCS data from a mixture is to use the decay times of the two species, so-called fluorescence lifetime correlation spectroscopy (Benda et al., 2006, Kapusta et al., 2007, Gregor \& Enderlein, 2007, Humpolíčková, et al. 2008). For the full and detailed overview about method and its applications one can read Rigler \& Elson (2001), Schwille \& Haustein (2001), Zander et al.,

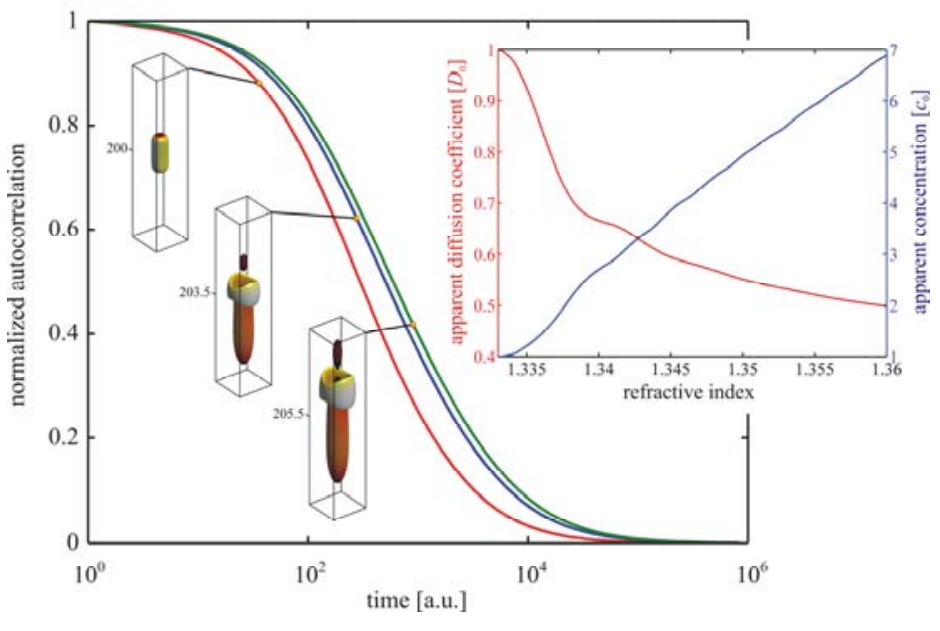

Fig.1.4: Wave-optical calculations concerning the effect of the molecule detection function on autocorrelation functions for different measurement conditions. The large figure shows, from left to right, the MDF and ACF for three increasing values of refractive index of the sample solution of $1.333,1.346$, and 1.360. The inset figure shows the dependence of apparent diffusion coefficient and the concentration on refractive index (Enderlein et al., 2005) (2002), Lacowicz (2006).

However, that wide range of FCS applications reveals its limitations. The most severe problem is a quantitative evaluation of an FCS measurement. For doing that, one

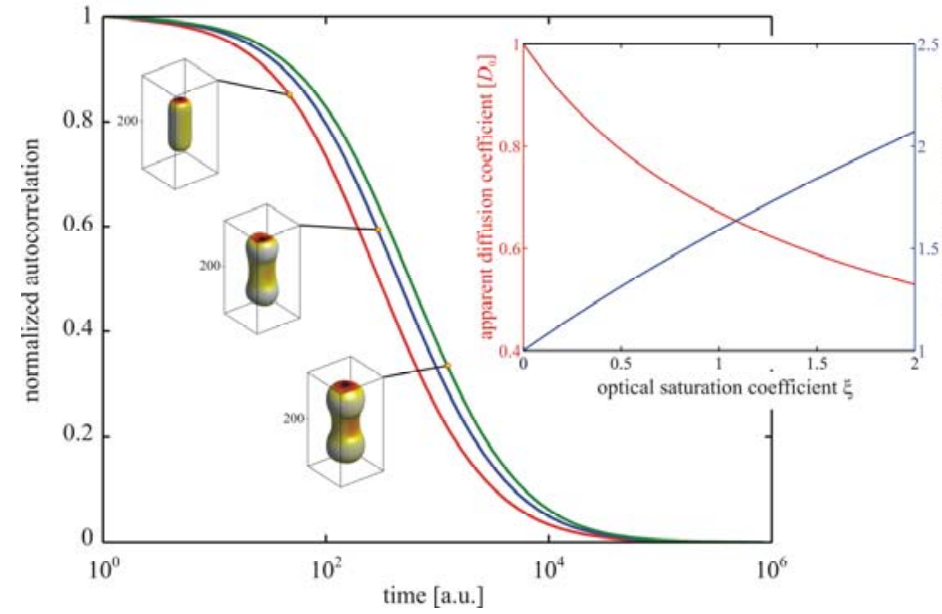

Fig.1.5: Wave-optical calculations concerning the effect of the molecule detection function on autocorrelation functions for different measurement conditions. The large figure shows, from left to right, the MDF and ACF for three increasing values of optical saturation. The inset figure shows the dependence of apparent diffusion coefficient and concentration on optical saturation i.e. excitation intensity (Enderlein et al., 2005). has to exactly know the size and the shape of the detection volume which is described by the so-called molecule detection function (MDF) giving the probability to detect a fluorescence photon from a molecule at a given position in sample space (Enderlein et al., 2004; Gregor et al., 2005). It sensibly depends on manifold parameters of the optical setup, such as the peculiarities of laser focusing or fluorescence light 
collection, which are difficult or impossible to control exactly. These dependencies make an exact and quantitative evaluation of FCS measurements rather difficult (Hess \& Webb, 2002; Nagy et al., 2005; Perroud et al., 2005). Furthermore, properties of the sample like refractive index, cover slide thickness, or laser beam astigmatism all influence the outcome of an FCS experiment (Enderlein et al., 2004; Enderlein, 2005). All those factors affect the shape and size of detection volume resulting in a change of the measured autocorrelation function (ACF) and thus extracted value of a diffusion coefficient. For example, even small deviation of sample refractive index from water, used as the immersion media for the most objectives in conventional FCS systems, cause a very complex shape of molecular detection function (Enderlein et al., 2004) and most importantly it leads to an increase of the detection volume and therefore to a dramatic decrease of the apparent diffusion coefficient (see Fig.1.4).

A reference measurement of fluorescent molecule, with an a priori known diffusion coefficient, under identical experimental conditions is usually used as a remedy for those problems. But even then, the most disturbing MDF problem in FCS measurements is the dependence of the MDF on excitation intensity due to optical saturation (Berland \& Shen, 2003, Nishimura \& Kinjo, 2004) (Fig.1.5). Thus the MDF depends on photophysical characteristics of each molecular species. Moreover photophysical properties of even the same dye often change when it is chemically bound to a protein or other target molecule (see, for example, (Eggeling et al., 2006)). This makes referential measurements problematic.

All those potential error sources are linked to a fundamental problem of FCS the absence of an intrinsic length scale in the measurement. The fluorescence correlation decay of the ACF depends on diffusion speed and the spatial extend and shape of the MDF, but the former is to be measured and the latter is not well known. The way to improve conventional FCS is to introduce an external parameter or ruler that is not prone to above mentioned optical and photophysical factors. Since 2002 several works have been published with proposal to modify optical excitation and/or detection schemes to achieve better definition and control of the MDF. Among them are z-scan FCS (Benda, et al., 2003, Humpoličková, et al., 2006), a method allowing for exact and absolute diffusion measurements in membranes, and scanning FCS (Ries \& Schwille, 2006, Petrasek \& Schwille, 2008), combining spatial and temporal correlation while scanning a focus in a well-defined manner. In particular, one tried to introduce an external ruler into the measurement, which is absent in standard FCS (Davis \& Bardeen, 2002; 
Rigneault \& Lenne, 2003; Jaffiol et al., 2006). The most successful and elegant modification is dual focus fluorescence correlation spectroscopy (2fFCS) (Dertinger, et $a l ., 2007 \& 2008)$. The neat idea, how extrinsic length-scale can be created and therefore absolute diffusion coefficient values can be obtained, is described next.

\subsection{Dual-focus Fluorescence Correlation Spectroscopy}

Dual-focus fluorescence correlation spectroscopy or $2 \mathrm{fFCS}$ is a special variation of FCS. In comparison with conventional single-focus FCS, a $2 \mathrm{fFCS}$ measurement records photon detection events from two detection volumes. Those volumes are identical but laterally shifted and overlapping with fixed centre distance between them. Exactly this distance defines an external invariable length scale in $2 \mathrm{fFCS}$ experiments (Dertinger et al., 2007). Many of the aforementioned experimental conditions such as laser beam quality or refractive index mismatch are be able to change the size and shape of the detection volume of each focus but not the centre distance between them. It makes 2fFCS largely insensitive to optical aberrations introduced by all those factors.

What makes $2 \mathrm{fFCS}$ very attractive for applications? This technique is relatively easy to implement. To introduce two identical foci in the sample space one has to use one extra laser emitting light of the same frequency but cross-polarized to another one and one more optical element - a Nomarski prism. This prism reflects laser beams under different angles according to their polarization and thus creates two identical foci in the sample space and defines the later shift between them. These are all the necessary modification of conventional single-focus FCS system.

Now, in $2 \mathrm{fFCS}$ experiments, photon detection events are recorded from two identical but laterally shifted detection regions. Therefore the ACFs for each detection volume as well as the cross correlation function (CCF) between the two detection volumes can be calculated. The CCF is calculated in a similar way as the ACF (Eq.1.1.) but correlating photons from different detection volumes:

$$
g(\tau)=\left\langle I_{1}(t) I_{2}(t+\tau)\right\rangle
$$

$I_{1}(t)$ is the fluorescence intensity collected from first focal volume at time $t$ and $I_{2}(t+\tau)$ is the intensity collected from the second one at time $t+\tau$, the triangular brackets denote time averaging. Thus, a typical output of $2 \mathrm{fFCS}-m e a s u r e m e n t$ consists of two 
autocorrelation- and one cross-correlation curve as shown in Fig.1.6. The CCF decays at longer times than ACFs because the fluorescent molecules have to pass the extra distance between both foci.

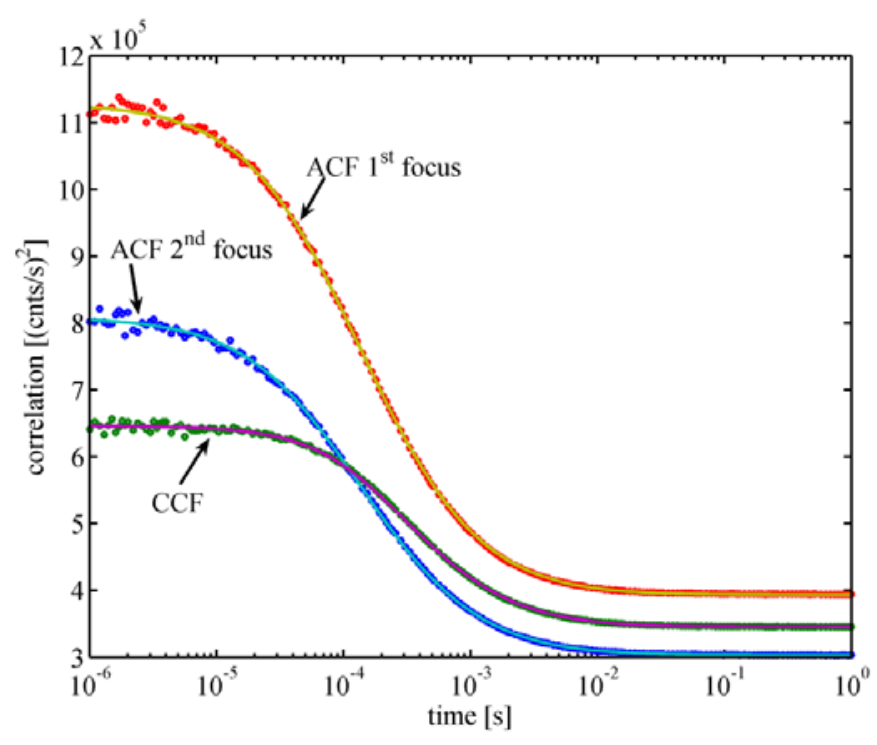

Fig.1.6: 2fFCS measurement on a nanomolar aqueous solution of Atto655. Shown are the autocorrelation functions for the first focus, the second focus, and the cross-correlation between both foci (CCF). The shape of both ACFs is virtually identical. Circles are experimental values; solid lines are global fits as described below.
The time delay of the cross correlation relative to the autocorrelation scales with the square of the distance between foci divided by the diffusion coefficient. Moreover, the relation between crosscorrelation to autocorrelation amplitude will be a direct measure of focus overlap. This leads to a very restrictive and thus stabilizing fit-criterion. Thus a global fitting applied to both auto- and cross-correlation curves yields an absolute value of the diffusion coefficient.

The 2fFCS robustness against optical aberrations caused by refractive index mismatch was demonstrated by Dertinger and co-authors (Dertinger et al., 2007). They measured diffusion coefficient of the red fluorescence dye Atto655 in aqueous solutions of guanidine hydrochloride. The concentration of guanidine hydrochloride was varied from zero to $6 \mathrm{M}$ within their experiments to increasing aberrations introduced by the refractive index mismatch. Increasing of guanidine hydrochloride concentration leads also to strong changes of the solutions' viscosity and therefore provides an excellent tool to visualize the aberrations influence on results. As according to the Stokes-Einstein relation between diffusion coefficient $D$, temperature $T$, solution viscosity $\eta$, and hydrodynamic radius $R_{\mathrm{h}}$ of the molecules,

$$
D=\frac{k_{B} T}{6 \pi \eta R_{h}}
$$


one would expect to find a linear relation between diffusion coefficient and in-verse of the viscosity when apparent and absolute diffusion coefficients agree with each other. The results are depicted on Fig.1.7. As can be seen, 2fFCS precisely yields the results predicted with the StokesEinstein relation. On the contrary,

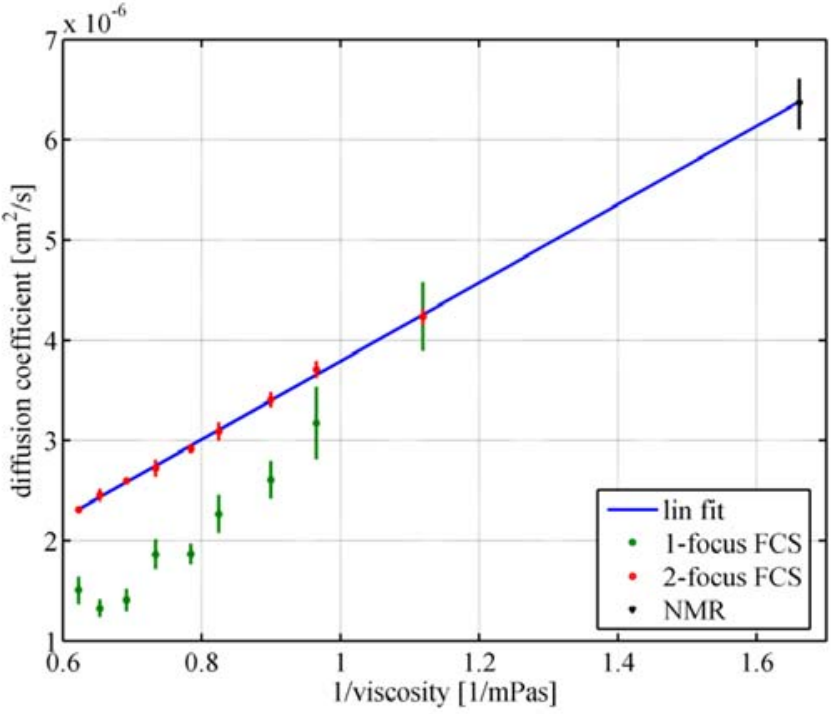

Fig.1.7: Dependence of the diffusion coefficient of Atto655 in aqueous guanidine hydrochloride solutions (red) and in d4-deuterized methanol (black) at $25^{\circ} \mathrm{C}$ as a function of solvent viscosity. Solid line is linear least square fit to all data. The results of single-focus FCS are shown in green (Dertinger et al., 2007).

Gaussian MDF, are strongly

affected by aberration and deviate

from linear dependence. Additionally the value of diffusion coefficient was determined with pulsed field gradient NMR, being capable of measuring absolute diffusion coefficients (Callaghan, 1991; Callaghan, 1999). It is shown in Fig.1.7, that the 2fFSC value of the diffusion coefficients perfectly match with that determined by pfgNMR.

To conclude, $2 \mathrm{fFCS}$ is able to measure absolute values of diffusion coefficient. The achievable accuracy of $2 \mathrm{fFCS}$ in diffusion coefficient measurements was estimated to be smaller than $5 \%$ (Dertinger et al., 2007). It opens the possibility to measure changes in the hydrodynamic radius of nanometer-sized molecules on the order of one Ångstrøm.

\subsection{Goals of the study}

The work presented here is concerned with high-precision sizing of molecules at pico- to nanomolar concentrations. Advanced variation of FCS, dual-focus FCS achieves the necessary accuracy to measure molecular size and moreover allows measuring absolute values of diffusion coefficient (and thus molecular size). Therefore this method is applied to determine size of different molecules.

The second chapter is concerned with methods and technical details. There I 
present a calibration procedure of centre distance between foci that is based on a combination of fluorescence correlation spectroscopy and dynamic light scattering. That is important as this distance introduces an external length scale into $2 \mathrm{fFCS}$ data evaluation. The accuracy in that distance determination defines how accurate the diffusion coefficient can be determined. I finish this chapter reporting diffusion coefficient values of three fluorescent dyes across the visible spectrum. Those values can be used as reference standards for fluorescence correlation spectroscopy.

Chapter 3 is focused on $2 \mathrm{fFCS}$ performance under optical saturation conditions. It includes theoretical study of $2 \mathrm{fFCS}$ performance with respect to systematical variations of experimental parameters such as laser focusing and laser excitation intensity. It is also shown experimentally that the method is robust against optical saturation within certain excitation intensity range. The experimental results are compared with conventional FCS measurements and theoretical prediction.

In chapter 4 the results of sizing small fluorescent molecules as well as globular proteins are presented. The sizing of three derivatives of Atto655 is used to demonstrate resolving power of the method. Then, I present results for chemically synthesized dyes, and for short oligopeptides. The observed quantitative relation between molecular weight and measured diffusion coefficient is discussed there. $2 \mathrm{fFCS}$ is used to size common globular proteins. The obtained values of hydrodynamic radius are compared with the published literature values and theoretical values calculated using the structural information from protein database.

In the last chapter I describe a novel method to measure rotational diffusion of large molecules (globular proteins) in solution based on fluorescence correlation on the nanosecond time scale. It is shown that the method works even if the rotational diffusion time is much longer than the fluorescence decay time. It is demonstrated that a pulsed interleaved excitation scheme with crossed excitation polarization maximizes the timedependent amplitude of the measured correlation curve as caused by rotational diffusion. Using the determined rotational diffusion coefficient, precise values of the hydrodynamic radius are obtained for four common globular proteins. 


\section{Experimental set-up and methods}

\subsection{Dual-focus Fluorescence Correlation Spectroscopy. Optical set-up}

The dual-focus or 2fFCS set-up is schematically shown in Fig. 2.1. It is based on an inverse epi-fluorescence microscope (MicroTime 200, PicoQuant, Berlin, Germany). The system is equipped with two identical pulsed $470 \mathrm{~nm}$ diode lasers (LDH-P-C-470B, PicoQuant, Berlin, Germany), two identical pulsed $635 \mathrm{~nm}$ diode lasers (LDH-P-635, PicoQuant, Berlin, Germany) with linear polarization. The duration of the laser pulses

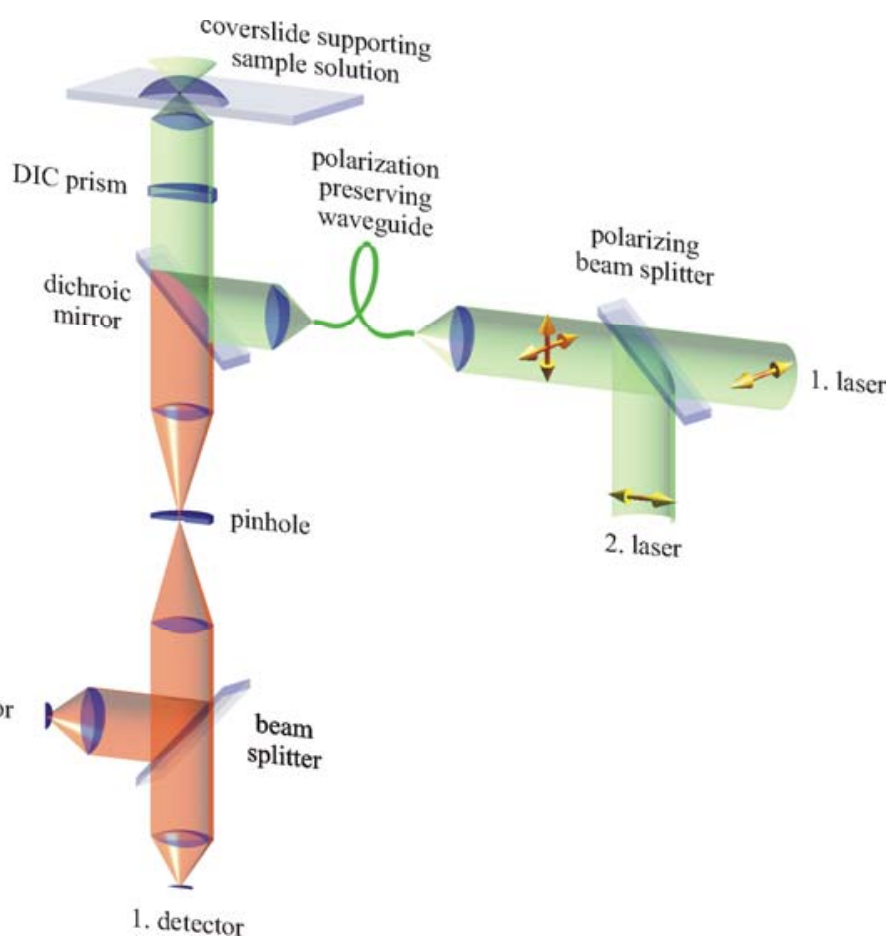

Fig.2.1: Schematic of the 2fFCS setup. is 50 ps (FWHM). The lasers are pulsed alternately with a sufficiently high repetition rate $(\sim 10-40 \mathrm{MHz})$, socalled pulsed interleaved excitation or PIE mode (Müller, B.K. et al., 2005)). Different repetition rates vary the delay between pulses from $25 \mathrm{~ns}$ to $100 \mathrm{~ns}$. The choice of the delay depends on the fluorescence lifetime of the label. It has to be long enough to assure that the fluorescence excited by one laser pulse decays completely before the next excitation pulse comes. Alternate pulsing is accomplished by special laser driver electronics (PDL 828 “Sepia II”, PicoQuant, Berlin, Germany).

The light of each of the two pairs of identical wavelength lasers is combined by two polarizing beam splitters (broadband polarizing cube by Ealing Catalogue, St. Asaph, UK) into single beams. The $635 \mathrm{~nm}$ and $470 \mathrm{~nm}$ beams are combined by a dichroic mirror (490 dcxr, AHF-Analysentechnik, Tübingen, Germany) resulting in a virtually single light beam containing both wavelengths with pulse trains of alternating polarization in each wavelength. The combined beam is optically shaped by sending it through a polarization-preserving single mode fiber. Before entering the back aperture of 
the water immersion objective (UPLAPO 60x W, 1.2 N.A., Olympus Europa, Hamburg, Germany), the light is passed through a Nomarski prism (U-DICTHC, Olympus Europa, Hamburg, Germany), see Fig.2.2, which is usually used for differential interference contrast (DIC) microscopy. This prism deflects the laser pulses into two slightly different directions depending on their polarization. After focusing through the objective, one thus obtains two overlapping foci with fixed lateral distance determined solely by the properties of the Nomarski prism, further called DIC prism.

Fluorescence is collected by the same objective (epi-fluorescence set-up), passed through the DIC prism and separated from the excitation light by a dichroic mirror $(\mathrm{Q}$ 660 LP, Chroma Technology, Rockingham, VT, USA).A tube lens focuses the light on to a single pinhole of $150 \mu \mathrm{m}$ diameter which is positioned symmetrically with respect to both focus positions. The pinhole is chosen large enough to let the light pass from both foci. After the pinhole, the light is re-collimated, split by a polarizing beam splitter cube (broadband polarizing cube by Ealing Catalogue, St. Asaph, UK), and refocused onto two single photon avalanche diodes (SPAD, AQR13, Perkin Elmer, Wellesley, MA, USA). The emission bandpass filters HC692/40 or HC520/35 (Semrock, USA) for red or blue excitation, respectively, positioned directly in front of each detector, discriminate fluorescence against scattered light.

A dedicated single-photon counting electronics (HydraHarp 400, PicoQuant Company, Berlin, Germany) can independently record the detected photons of both detectors with an absolute temporal resolution of one picosecond. By evaluating the arrival times of the photons on a nanosecond time scale, the detected photons can be unequivocally associated with its corresponding excitation pulse and thus with the corresponding focus. Thus, it is possible to calculate the auto correlation functions (or $\mathrm{ACFs}$ ) for each focus separately, as well as the cross-correlation function (or CCF) between photons emerging from both foci.

Besides, only photons from the two different detectors are correlated to prevent distortions of the resulting ACF by SPAD afterpulsing, which is the effect of faked photon generation and detection after a true photon detection event (Enderlein \& Gregor, 2005). 


\subsection{Calculating the auto- and cross-correlation functions}

A 2 fFCS measurement records photon detection events from two identical but laterally shifted detection regions. Thus in a $2 \mathrm{fFCS}$ measurement, two ACFs are calculated, for each detection volume separately. The autocorrelation $\langle i(t) i(t+\tau)\rangle$ of the fluorescence signal from one and the same molecule can be easily derived when remembering its physical meaning: It is proportional to the chance to see, from one and the same molecule, a photon at time $t+\tau$ if there was a photon detection at time $t$. The probability to find a molecule within an infinitely small volume $d V$ anywhere in the sample is equal to $d V / V$, where $V$ is the total sample volume. Next, the probability to detect a photon from a molecule at a given position $r_{1}$ is directly proportional to the value of the molecular detection function (or MDF) at this position, i.e. to $U\left(\vec{r}_{1}\right)$. Furthermore, the chance that the molecule diffuses from position $\vec{r}_{1}$ to position $\vec{r}_{2}$ within time $\tau$ is given by the solution of the diffusion equation for the given initial (molecule at position $\vec{r}_{1}$ ) and boundary conditions. For a sample with distant boundaries this solution has the simple form:

$$
G(\vec{r}, \tau)=\frac{1}{(4 \pi D \tau)^{3 / 2}} \exp \left(-\frac{\left|\vec{r}_{2}-\vec{r}_{1}\right|^{2}}{4 D \tau}\right)
$$

where $D$ is the diffusion coefficient of the molecule. Finally, the chance to detect a photon from the molecule at the new position is again proportional to the value of the MDF at this position, i.e. to $U\left(\vec{r}_{2}\right)$. Thus, the autocorrelation $g(t)$ is calculated as the product of all these individual contributions and averaging over all possible initial and final positions of the molecule, i.e. integrating over $\vec{r}$ :

$$
g(t)=c \varepsilon_{1,2}^{2} \int_{V} d \vec{r}_{1} \int_{V} d \vec{r}_{2} U\left(\vec{r}_{2}\right) \frac{1}{(4 \pi D t)^{3 / 2}} \exp \left(-\frac{\left|\vec{r}_{2}-\vec{r}_{1}\right|^{2}}{4 D t}\right) U\left(\vec{r}_{1}\right)
$$

where $c$ is the concentration of the molecules; $\varepsilon_{1,2}$ are two factors describing the two different overall detection efficiency in both detection volumes, respectively.

The CCF is calculated in a similar way as the ACF but correlating photons from 
the two different detection volumes and taking into account the spatial separation between them. Let's assume that the lateral shift is along the $x$-axis perpendicular to the optical axis of the microscope. Then expression for CCF can be written in the same form as Eq.2.2 for one-focus FCS:

$$
g(t, \delta)=\varepsilon_{1} \varepsilon_{2} c \int d r_{1} \int d r_{2} U\left(r_{2}\right) \frac{1}{(4 \pi D t)^{3 / 2}} \exp \left[-\frac{\left(r_{1}-r_{2}-\hat{x} \delta\right)^{2}}{4 D t}\right] U\left(r_{1}\right)
$$

where the vector $\hat{x}$ is the unit vector along $x, \delta$ is the lateral shift value.

A crucial point for a successful $2 \mathrm{fFCS}$ data analysis is to have a sufficiently appropriate model function for the MDF. Recently, Dertinger and coauthors showed that the MDF of a confocal microscope can be fairly well-approximated by a GaussLorentzian function (Dertinger et al., 2007):

$$
U(\vec{r})=\frac{\kappa(z)}{w^{2}(z)} \exp \left(-\frac{2}{w^{2}(z)}\left[\left(x \pm \frac{\delta}{2}\right)^{2}+y^{2}\right]\right)
$$

where $x$ and $y$ are transversal coordinates perpendicular to the optical axis $z=0$. Eq.2.4 is a modification of the three-dimensional Gaussian and says that in each plane perpendicular to the optical axis, the MDF is approximated by a Gaussian distribution having width $w(z)$ and amplitude $\kappa(z) / w^{2}(z)$. The functions $\kappa(z)$ and $w(z)$ are given by

$$
w(z)=w_{0}\left[1+\left(\frac{\lambda_{e x} z}{\pi w_{0}^{2} n}\right)^{2}\right]^{1 / 2}
$$

and

$$
\kappa(z)=1-\exp \left(-\frac{2 \alpha^{2}}{R^{2}(z)}\right)
$$

where the function $R(z)$ is defined by: 


$$
R(z)=R_{0}\left[1+\left(\frac{\lambda_{e m} z}{\pi R_{0}^{2} n}\right)^{2}\right]^{1 / 2}
$$

Here, $\lambda_{e x}$ is the excitation wavelength, $\lambda_{e m}$ is the center emission wavelength, $n$ is the refractive index of the immersion medium (water), $\alpha$ is the radius of the confocal aperture divided by magnification, and $w_{0}$ and $R_{0}$ are two (generally unknown) model parameters. Eq.2.5 is nothing else than the scalar approximation for the radius of a diverging laser beam with beam waist radius $w_{0}$.

Using MDF given by Eq.2.4 as well as the parameterisation given by Eqs. 2.5 through 2.7, Eq.2.3 for calculating cross-correlation curve of the two-focus set-up can be written as

$$
\begin{aligned}
& g(t, \delta)=\frac{\varepsilon_{1} \varepsilon_{2} c}{4} \sqrt{\frac{\pi}{D t}} \int_{-\infty}^{\infty} d z_{1} \int_{-\infty}^{\infty} d z_{2} \frac{\kappa\left(z_{1}\right) \kappa\left(z_{2}\right)}{8 D t+w^{2}\left(z_{1}\right)+w^{2}\left(z_{2}\right)} \cdot \ldots \\
& \exp \left[-\frac{\left(z_{2}-z_{1}\right)^{2}}{4 D t}-\frac{2 \delta^{2}}{8 D t+w^{2}\left(z_{1}\right)+w^{2}\left(z_{2}\right)}\right]
\end{aligned}
$$

Data fitting is performed by least-square fitting of the model curve, Eq.2.8, against the measured ACFs and cross-correlation CCF simultaneously in a global fit. As fit parameters, one has $\varepsilon_{1} c / 2, \varepsilon_{2} c / 2, D, w_{0}$ and $R_{0}$. The distance $\delta$ between the detection regions is determined by the properties of the DIC prism and has to be exactly known $a$ priori, thus introducing an external length scale into data evaluation.

\subsection{Calibration of the shear distance of the Nomarski-prism}

A Nomarski prism, also called differential-interference contrast or DIC prism, is mostly used in differential interference contrast microscopy (Nomarski,G., 1970). The DIC prism, which is a modified Wollaston prism, consists of two optical quartz or calcite wedges cemented together at the hypotenuse. One of the wedges is identical to a conventional Wollaston quartz wedge and has the optical axis oriented parallel to the surface of the prism (Fig.2.2). The second wedge of the prism is modified by cutting the quartz crystal in such a manner that the optical axis is oriented obliquely with respect to the flat surface of the prism. The Nomarski modification causes the light rays to come to 
a focal point outside the body of the prism and thus allows greater flexibility when setting up the microscope. In $2 \mathrm{fFCS}$, the DIC prism is one of the core elements in the measurement set-up that generates two laterally shifted but overlapping detection volumes. The value of that shift is fixed and well defined. Therefore the DIC prism constructs a spatial ruler for precise and qualitative determination of diffusion coefficients (Dertinger et al., 2007).

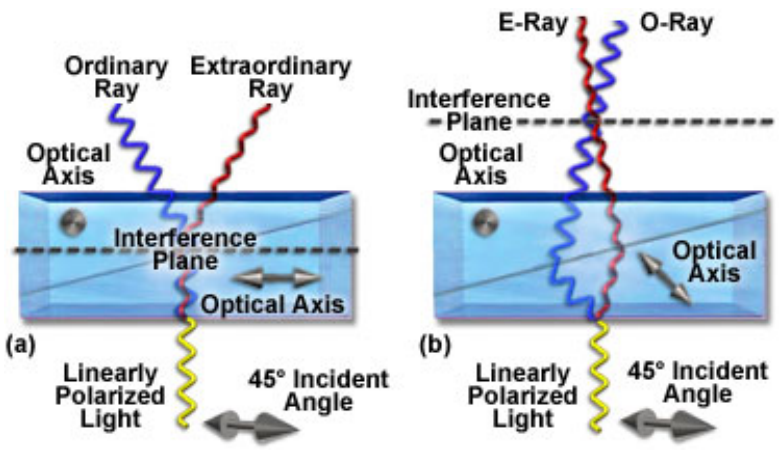

Fig.2.2: Schematic of Wollaston (a) and Nomarski (b) prisms in differential interference contrast (DIC) microscopy. Ordinary and extraordinary rays correspond to laser beams with different polarization. The paths of beams with parallel and orthogonal polarization are depicted for Wollaston and Nomarski prisms (www.microscopyu.com)

The precision with which the interfocal distance is known determines the accuracy with which a diffusion coefficient can be measured as its value scales with the square of the adopted focus distance. For example, for an interfocal distance of $500 \mathrm{~nm}$, one has to know the distance with an accuracy of better than $10 \mathrm{~nm}(2 \%)$ when aiming for an accuracy of the diffusion coefficient measurement of better than $4 \%$. And vice versa when the diffusion coefficient or hydrodynamic radius is known with high precision, one can use this value to quantify the distance between the two propagating light beams generated by the DIC prism. Thus the core idea is to (i) measure with dynamic light scattering (DLS) the hydrodynamic size of commercially available fluorescently labelled and monodisperse spherical colloidal latex particles, and (ii) to measure with $2 \mathrm{fFCS}$ the diffusion coefficient of these particles. By comparing the hydrodynamic radius as obtained with both methods, one can directly determine the distance between the detection volumes in the 2fFCS measurement set-up, and thus the shear distance of the DIC prism. This strategy is presented in the paper published together with Müller,C.B. (2008a).

The work presented in this thesis was done on a system implemented with four pulsed excitation lasers at $470 \mathrm{~nm}$ and $640 \mathrm{~nm}$. The shear distance of the DIC prism is wavelength-dependent because of the DIC material dispersion and therefore has to be determined for each wavelength. 


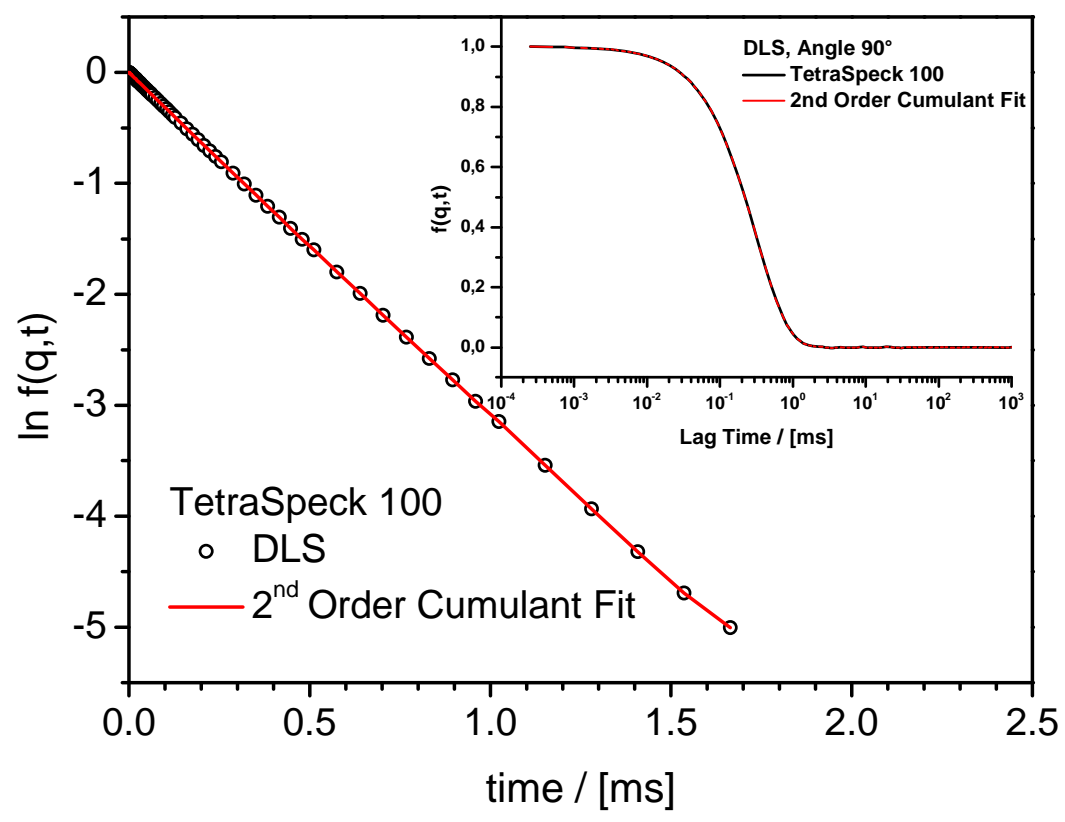

Fig.2.3: Dynamic light scattering measurements. Main picture: DLS at $90^{\circ}$ of mono disperse TetraSpeck 100 latex particles, fitted with a $2^{\text {nd }}$ order cumulant fit. Inset: standard plot of ACF (Müller,C.B. et al., 2008a).

The smallest multi-fluorescent TetraSpeck beads of $100 \mathrm{~nm}$ in diameter were used for the calibration. They can be still considered as point source of light in $2 \mathrm{fFCS}$ experiments (Müller, et al., 2008b) but are big enough to get a good signal to noise ratio in dynamic light scattering measurements. DLS is used to determine the beads size and the corresponding diffusion coefficient. Measurements were performed on a "Coulter N4 Plus" DLS system (Beckman Coulter Inc., Brea, USA) using a detection angle of $90^{\circ}$. The temperature was kept at $293.15 \pm 0.1 \mathrm{~K}$ in all DLS experiments. The measurements were done with excitation light of $640 \mathrm{~nm}$ and were repeated thirty times to get a sufficiently small standard deviation. A semi-logarithmic plot of the data is shown in the main panel of Fig.2.3, together with a second order cumulant fit. The good fit quality proves the good monodispersity of the bead sample. The hydrodynamic radius $R_{h}$ of the beads was determined to be $55.4 \pm 0.6 \mathrm{~nm}$. This result is in a good agreement with value provided by company and equal to $50 \pm 5 \mathrm{~nm}$.

In a second step, 2fFCS measurements were performed at the two excitation wavelengths of $470 \mathrm{~nm}$ and $640 \mathrm{~nm}$, respectively. Due to the high label density of the beads, total excitation power was reduced to less than $0.1 \mu \mathrm{W}$ within each detection volume. A few correlation functions had to be discarded due to distortions generated by 
the transit of large particle clusters through the detection volume. A typical measurement result is shown in the inset of Fig.2.4.

To reveal the distance between the overlapping detection volumes, each set of ACFs and CCF was globally fitted by the model function of Eq.2.8 to obtain a value of the diffusion coefficient $D$ and thus hydrodynamic radius $R_{h}$. The fits were done for a series of assumed distances $\delta$ between the detection volumes between 360 and $416 \mathrm{~nm}$.

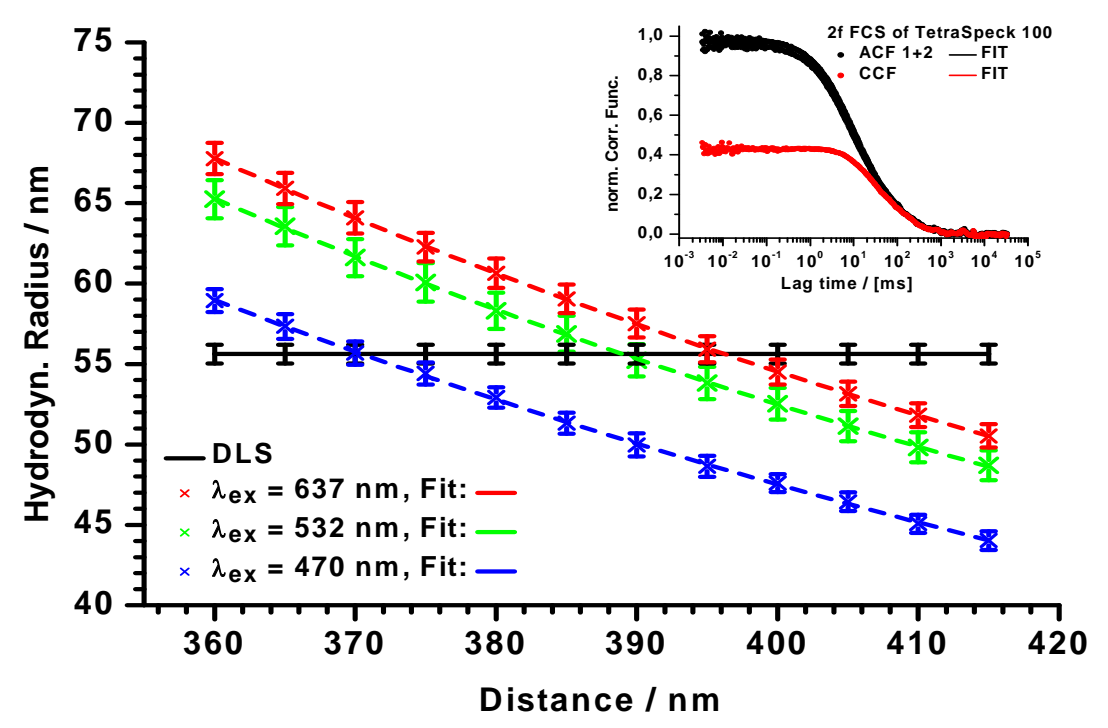

Fig. 2.4: Main picture: Wavelength dependent determination of DICprism shear distance, by comparison of DLS and $2 \mathrm{f}$-FCS measurements, obtained from enhanced model for multi labeled particles. Inset: 2fFCS measurement of TetraSpec 100 latex particles. Autocorrelation (ACF) and cross-correlation (CCF) functions, fitted with 2fFCS model Eq.2.8 (Müller,C.B. et al., 2008a).

The obtained hydrodynamic radii $R_{h}$ as a function of assumed distance $\delta$ is shown in Fig.2.4. The intersection of this curve with a horizontal line at the actual value of the hydrodynamic radius as obtained from the DLS measurements gives the actual distance between the detection volumes, and thus the shear distance of the DIC prism. Table 2.1 lists the obtained values of the shear distance at the two different excitation wavelengths. Standard deviation of the $2 \mathrm{fFCS}$ measurements is better than $0.4 \%$, or \pm $1.5 \mathrm{~nm}$. 
Table 2.1: Wavelength dependent shear distances obtained from comparison of DLS and 2fFCS experiments

Excitation wavelength $\left(\lambda_{e x}\right)$

$470 \mathrm{~nm}$

$640 \mathrm{~nm}$
Shear distance of DIC prism

$395 \mathrm{~nm}$

$414 \mathrm{~nm}$

\subsection{Absolute and precise diffusion coefficient measurements across the visible} spectrum

One of the significant disadvantages of a conventional single-focus FCS measurement is that it has always to be referenced against a standard sample with known diffusion coefficient. The typical reference sample is an aqueous solution of the dye Rhodamine 6G (Rh6G), and for over three decades its diffusion coefficient was reported as $D_{22^{\circ} \mathrm{C}}(R h 6 G)=(2.8 \pm 0.7) \cdot 10^{-6} \mathrm{~cm}^{2} / \mathrm{s}$ in a buffer solution of $10^{-4} \mathrm{M} \mathrm{Na}$ ethylenediaminetetraacetic acid, $10^{-1} \mathrm{M} \mathrm{NaCl}$ and $10^{-2} \mathrm{M}$ tris-(hydroxymethyl)aminomethane at $8.0 \mathrm{pH}$ (Madge et al., 1974). In spite of the knowledge that the diffusion coefficient of a substance depends on solvent viscosity (for example see Madge et al., 1974), in most publications that value is used directly for aqueous solutions of Rh6G, where it translates into a value of $D_{25^{\circ} \mathrm{C}}(R h 6 G)=(3.0 \pm 0.8) \cdot 10^{-6} \mathrm{~cm}^{2} / \mathrm{s}$ at $25^{\circ} \mathrm{C}$. Using advantage of $2 \mathrm{fFCS}$ to measure absolute and precise values of diffusion coefficient, we have measured the new diffusion coefficient value of Rh6G and diffusion coefficients of two more widely used fluorescence dyes that can serve as diffusion standards. The results of that work are reported here and published together with Müller C.B. (2008c).

We have chosen the three widely used fluorescent dyes Oregon Green ${ }^{\circledR} 488$, Rhodamine 6G, and Atto655-maleimide that are excitable at three different wavelengths $470 \mathrm{~nm}, 532 \mathrm{~nm}$, and $637 \mathrm{~nm}$ to cover the most used part of the visible spectrum where FCS experiments are performed. Their chemical structures are depicted on Fig.2.5. All dyes were measured in aqueous solutions. $2 \mathrm{fFCS}$ measurements for Rhodamine $6 \mathrm{G}$ were done in collaboration with Claus Bernd Müller, at that time at the Institute of Physical Chemistry of the RWTH Aachen University (Germany). In all cases it was checked that the obtained diffusion coefficient was independent on excitation intensity, in stark 
contrast to typical single-focus FCS measurements. Only when the excitation power per focus was exceeding ca. $50 \mu \mathrm{W}$ photobleaching started to accelerate the apparent diffusion coefficient.
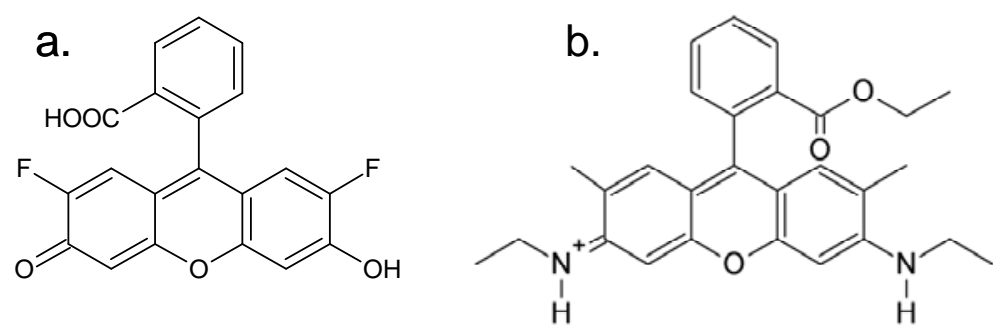

C.<smiles></smiles>

Fig.2.5: Chemical structure of fluorescence dyes: Oregon Green 488 (a), Rhodamine 6G (b) and MR121 (c). MR121 belongs to the same group of oxazine molecules as Atto655 and has similar structure.

Let's start from already mentioned Rh6G. It is known that the grade of purity is quite different for commercially available rhodamine. Thus Rh6G was obtained from two different suppliers: No. 83697 from Sigma-Aldrich (Seelze, Germany), and R634 from Invitrogen (Karlsruhe, Germany). The purity of the samples was checked using high performance liquid chromatography (HPLC).

Rh6G purchased from Sigma-Aldrich shows a purity grade of less than 55\%, but the remainder absorbs also at $532 \mathrm{~nm}$ and exhibits fluorescence. The obtained value of $D_{25^{\circ} \mathrm{C}}(R h 6 G)=(3.89 \pm 0.3) \cdot 10^{-6} \mathrm{~cm}^{2} / \mathrm{s}$ corresponds to the average diffusion coefficient of that mixture and can not be used as a calibration standard.

Rhodamine $6 \mathrm{G}$ purchased from Invitrogen showed a purity grade of better than 95\%, as checked with HPLC. Its diffusion coefficient $D_{25^{\circ} \mathrm{C}}(R h 6 G)=(4.14 \pm 0.05) \cdot 10^{-6} \mathrm{~cm}^{2} / \mathrm{s}$ corresponds to a hydrodynamic radius of $5.89 \AA$ and is in perfect agreement with a recently published measurement using plug broadening in capillary flow (Culbertson et al., 2002). Therefore, this value can be recommended for calibration. It is important to note that the found value of the diffusion coefficient is by $37 \%$ larger than the previously reported value for that dye, used as the reference standard in single-focus FCS measurements for many decades.

For the dye Atto655-maleimide (AttoTec, Siegen, Germany), a diffusion coefficient in water $D_{25^{\circ} \mathrm{C}}($ Atto655-maleimide $)=(4.07 \pm 0.1) \cdot 10^{-6} \mathrm{~cm}^{2} / \mathrm{s}$ was found. A 
cross-check experiment was made for the same dye in deuterized methanol (methanol4d) with pfgNMR. The Stokes-Einstein relation was applied to calculate the dye hydrodynamic radius. In both solvents, the value of the hydrodynamic radius was found to be identical (within our measurement errors) and equal to $(6.01 \pm 0.11) \AA$.

Finally, the diffusion coefficient of the dye 2', 7'- difluorofluorescein (Oregon Green $^{\circledR}$ 488) (No. D6145, Invitrogen, Karlsruhe, Germany) was found to be $D_{25^{\circ} \mathrm{C}}($ Oregon Green 488$)=(4.11 \pm 0.06) \cdot 10^{-6} \mathrm{~cm}^{2} / \mathrm{s}$, corresponding to a hydrodynamic radius of $5.95 \AA$.

The measured diffusion coefficients of all three dyes are summarized in to the following table:

Table 2.2: Diffusion coefficients of fluorescent dyes across the visible spectrum.

Fluorescent dye

Oregon Green 488

Rhodamine $6 \mathrm{G}$

Atto655 maleimide

$$
D_{25^{\circ} \mathrm{C}} / 10^{-6} \mathrm{~cm}^{2} / \mathrm{s}
$$

$$
4.11 \pm 0.06
$$

$4.14 \pm 0.05$

$4.07 \pm 0.10$ 



\section{Performance of dual-focus Fluorescence Correlation Spectroscopy under optical saturation}

At the beginning of this chapter I introduce optical saturation phenomenon. It is followed by the theoretical study of $2 \mathrm{fFCS}$ performance under optical saturation. At the end I will present experimental results of optical saturation effect in 2fFCS.

\subsection{Optical saturation}

The most important and most disturbing source of inaccuracy and irreproducibility in conventional FCS measurements is the dependence of the ACF decay alias diffusion time on the excitation intensity due to optical saturation of fluorescence. In contrast to other optical artifacts of conventional FCS, optical saturation makes even comparative measurements of diffusion coefficients problematic because the fluorescence properties of many fluorescing dyes used for labeling of proteins, DNA, or RNA, and thus their optical saturation behavior, are changing upon binding to these molecules.

Optical saturation occurs when the excitation intensity becomes so large that a molecule spends more and more time in a non-excitable state, so that increasing the excitation intensity does not lead to a proportional increase in emitted fluorescence intensity. The most common sources of optical saturation are (i) excited state $\left(\mathrm{S}_{1}\right)$ saturation, i.e. the molecule is still in the excited state when the next potential excitation photon arrives; (ii) triplet state saturation, i.e. the molecule undergoes intersystemcrossing from the excited to the triplet state so that it can no longer become excited until it returns back to the ground-state; (iii) other photoinduced transitions into a nonfluorescing state, such as the photoinduced cis-trans-isomerization in cyanine dyes, or the optically induced dark states in quantum dots.

The exact relation between fluorescence emission intensity and excitation intensity can be very complex and even dependent on the excitation mode (pulsed or continuous wave) (Gregor et al., 2005). Moreover, the rate of change of apparent diffusion coefficient with increasing excitation intensity is largest in the limit of infinitely small intensity (Gregor et al., 2005; Enderlein et al., 2005). Therefore, for precise diffusion coefficient measurements it is necessary to repeat the measurement at different excitation intensities and to extrapolate the obtained diffusion coefficients towards zero excitation intensity. 
What about dual-focus FCS? How does 2fFCS perform under optical saturation? These questions will be answered in the present chapter.

The theoretical study of the performance of $2 \mathrm{fFCS}$ can be found in a paper published together with Dertinger et al. in 2008. Here, I will present the main results of these calculations concerning optical saturation, and I will compare the theoretical predictions with experiments. The experimental part of this chapter was published in Loman et al. in 2008.

\subsection{Theoretical study}

Calculations of the molecular detection function or MDF and subsequent calculation of the ACF and CCF curves were performed for different laser focus sizes. The considered range of focus size started with a rather large value corresponding to a $1.25 \mathrm{~mm}$ beam-radius (relaxed focusing), and did end with a focus size close to the diffraction limit which corresponds to a $4 \mathrm{~mm}$ laser beam-radius (there is an inverse relationship between laser beam diameter at the back-focal plane of the objective and focus diameter in the sample). The calculated ACF/CCF curves were then fitted with Eq.2.8 that was derived by assuming a Gauss-Lorentz model for the MDF (Eqs. 2.3 through 2.7), as described in chapter 2. These are the same equations as used for real 2fFCS measurements analysis. For more details see Dertinger et al., 2008, and references therein.

Let's start by considering the simplest case of optical saturation connected with the $S_{0} \rightarrow S_{1}$ transition and the finite lifetime of the excited state. The relevant parameter determining the degree of optical saturation is the ratio of average excitation rate to saturation intensity $I_{s a t}=\left(\sigma \cdot \tau_{f}\right)^{-1}$ (given here in units of photons per area per time), where $\sigma$ denotes the molecules' absorption cross section at the excitation wavelength (Gregor et al., 2005), and $\tau_{f}$ is the excited state lifetime. In our modeling, this ratio, at the position of maximum intensity (cross point of optical axis with focal plane), was varied between zero and one, which means that the maximum excitation intensity in the very centre of the focus was varied between zero and $I_{\text {sat }}$.

The impact of varying saturation on the apparent diffusion coefficient $\left(D_{f i t}\right)$ is shown in Fig.3.1. There, I compare the sensitivity of $2 \mathrm{fFCS}$ against $\mathrm{S}_{0} \rightarrow \mathrm{S}_{1}$ optical saturation for different degrees of focusing. As can be seen in Fig.3.1, for rather relaxed focusing (laser beam radius below $\sim 2 \mathrm{~mm}$ ), diffusion coefficients ratio $D_{\text {fit }} / D$ is rather insensitive to 


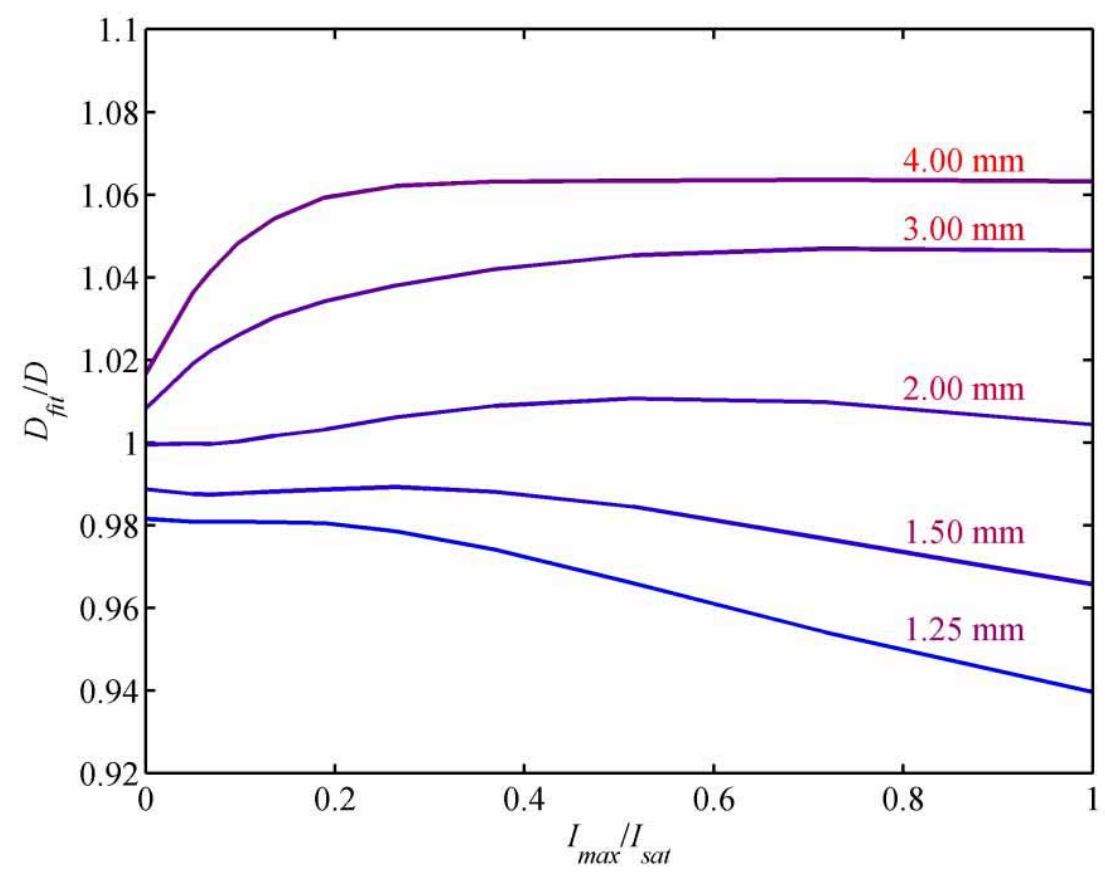

Fig.3.1: The sensitivity of 2 fFCS against $S_{0} \rightarrow S_{1}$ optical saturation for different degrees of focusing. Shown are the global fit results of a $2 \mathrm{fFCS}$ measurement with five different laser beam radii between 1.25 and $4 \mathrm{~mm}$ (Dertinger et al., 2008).

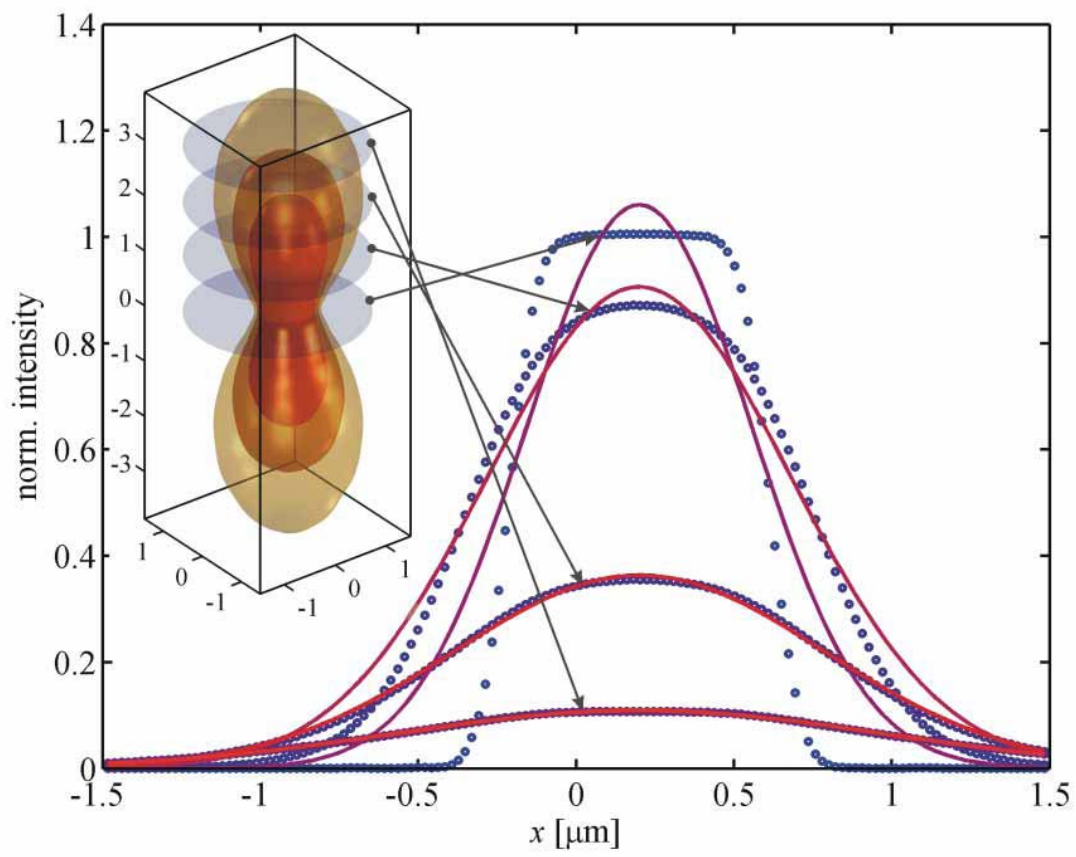

Fig.3.2: Anatomy of the MDF for focusing a laser beam with radius $R=2$ $\mathrm{mm}$ and $\mathrm{S}_{0} \rightarrow \mathrm{S}_{1}$ optical saturation parameter of one (Dertinger et al., 2008). 
saturation as long as the maximum excitation intensity remains below $\sim 0.2 \cdot I_{\text {sat }}$, and focusing is not too tight. Under optimal conditions (laser beam radius is equal to $2 \mathrm{~mm}$ ), the extracted diffusion coefficient deviates by less then $1 \%$ from its actual value. And even for the two extreme cases of focusing, the relative error is not larger than $7 \%$. This is in stark contrast to conventional single-focus FCS as was analyzed in Enderlein et al. (2005).

The robustness of $2 \mathrm{fFCS}$ against optical saturation is remarkable in the light of that it affects the shape and size of the MDF, of course. In Fig. 3.2 it is shown how much the MDF is deformed at the extreme saturation value of one, i.e. when the maximum excitation intensity in each focus is equal to $I_{\text {sat }}$. This changes the overlap of the two foci as well as the relative amplitude of the CCF to that of the ACF. However, because the distance between the foci centres does not change, a global fit of ACF and CCF can mostly compensate for the effects introduced by saturation and yields sufficient accurate values of the diffusion coefficients. Typical examples for the fit quality of the fitting curves Eq.2.8 against modeled $\mathrm{ACF} / \mathrm{CCF}$ curves is shown in Fig.3.3.

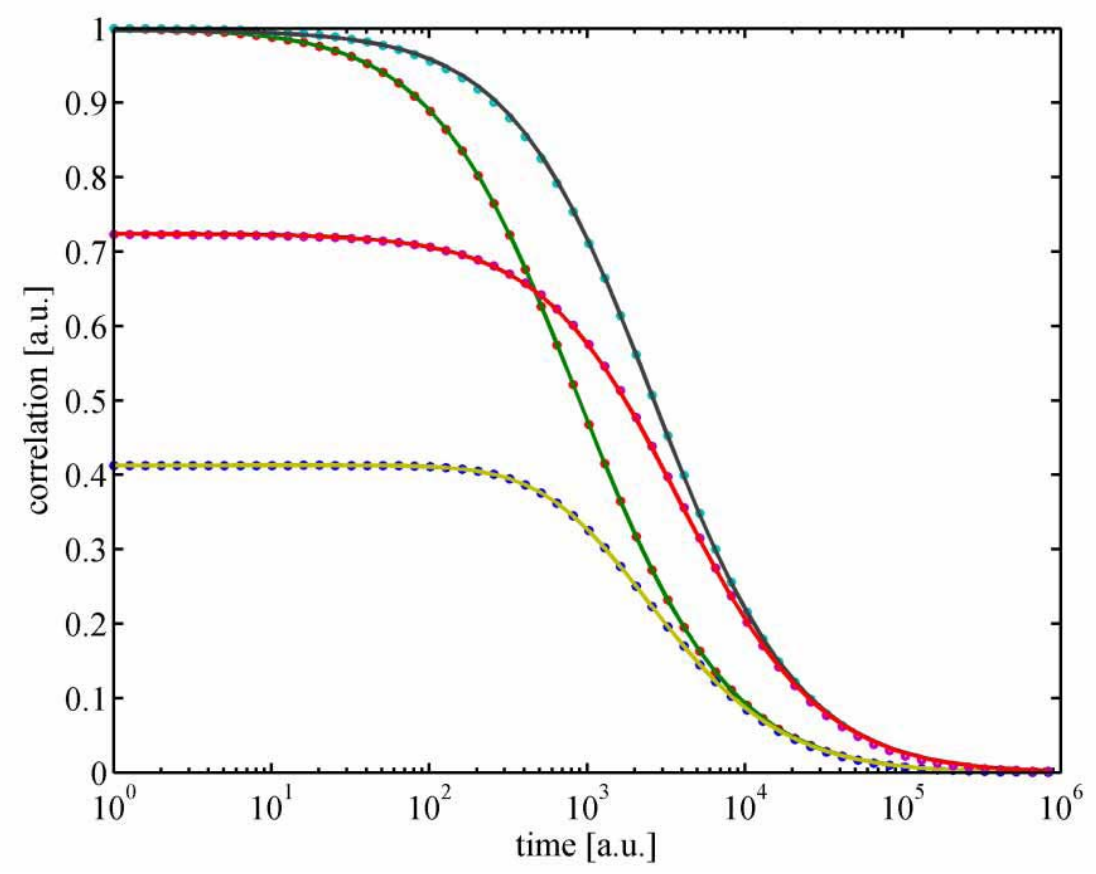

Fig.3.3: Fit quality of the global fit of a 2fFCS experiment under ideal optical conditions (left couple of curves) and for a $S_{0} \rightarrow S_{1}$ optical saturation of one (right couple of curves). Dots are the theoretically calculated auto- and crosscorrelation curves; solid lines are the best global fit (Dertinger et al., 2008). 


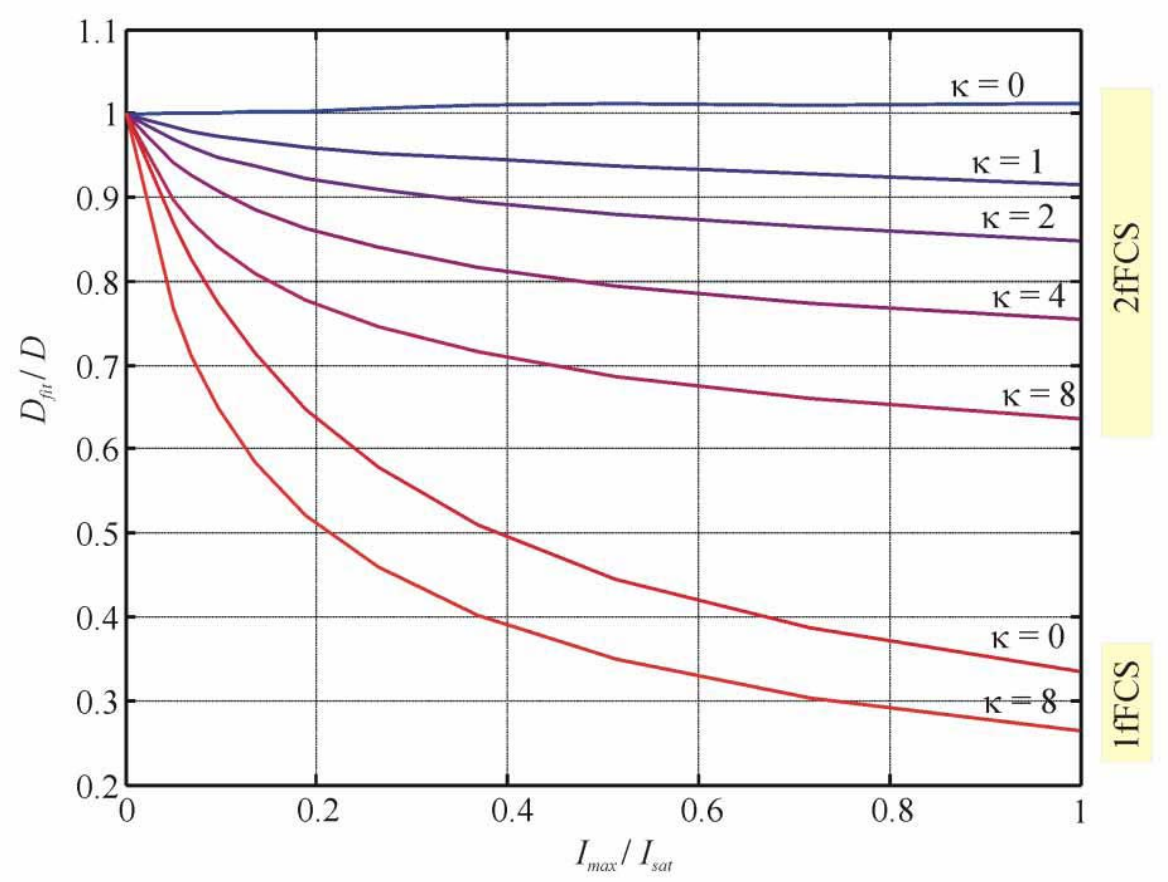

Fig. 3.4: Dependence of the fitted absolute value of the diffusion coefficient on excited state saturation with different ratios $\kappa$ of intersystem crossing rate constant to phosphorescence rate constant, i.e. $k_{i s c} / k_{p h}$. Shown are the global fit results of a $2 \mathrm{fFCS}$ measurement assuming a laser beam radius of $2 \mathrm{~mm}$. For comparison, fit results from a single-focus FCS measurement are also shown, for the two limiting $\kappa$-values of 0 (no triplet state dynamics, compare with Fig.3.1) and 8. The two single-focus FCS curves are normalized by their value at zero optical saturation, i.e. in the limit of zero excitation intensity (Dertinger et al., 2008).

Next, I will focus on the more complex mechanisms of saturation when molecules are driven into a non-fluorescent triplet state or into some other non-fluorescent conformation. The relevant parameter, in this case, is the ratio between intersystem-crossing rate constant $k_{\text {isc }}$ and triplet-state-relaxation rate constant $k_{p h}$, i.e. $\kappa=k_{\text {isc }} / k_{\text {ph }}$. Fig.3.4 represents the impact of triplet state pumping and relaxation to the performance of conventional FCS and 2fFCS for the optimal laser beam radius of $2 \mathrm{~mm}$. The results are compared as a function of excitation intensity in units of $I_{\text {sat }}$ for different values of $\kappa$. As can be seen now, with increasing triplet state pumping efficiency the outcome of a $2 \mathrm{fFCS}$ measurement in terms of a determined diffusion coefficient becomes more and more biased towards smaller values with increasing excitation intensity, although the sensitivity is still not as large as in the case of conventional FCS.

In the current versions of $2 \mathrm{fFCS}$ using pulse interleaved excitation or PIE, the excitation between foci is switched with a high repetition rate much faster than the typical triplet state transition and relaxation rates, so that the slow photophysical 
dynamics "sees" only an average excitation which is the sum of the excitation intensity distributions in each focus. Thus, in the region between foci the excitation intensities sum up, leading to an apparent pushing-away of the centres of the two MDFs, which makes their effective distance larger than it is assumed from the properties of the DIC prism. The effect is getting stronger with increasing saturation, as soon as the photophysical processes behind the saturation are much slower than the time between alternate pulsing in the PIE excitation scheme. However, PIE excitation can also be used for circumventing this problem: the waiting time between laser pulses can be chosen to be longer than the triplet state relaxation time. In this way, an apparent pushing-away of the centres of the two overlapping MDFs can be avoided. This will result in a similar insensitivity of the determined diffusion-coefficient on excitation intensity as was seen for the pure $\mathrm{S}_{0} \rightarrow \mathrm{S}_{1}$ saturation state (Donnert et al., 2007). Another option is to use reducing/oxidizing (ROXS) or Trolox chemistry that rapidly depletes the triplet state (Vogelsang et al.,2008; Cordes et al., 2009).

\subsection{Cy5 diffusion under optical saturation}

The widely used cyanine dye Cy5 is used to check the performance of $2 \mathrm{fFCS}$ under optical saturation. Cy5 exhibits a strong light-driven cis-trans transitions between fluorescent and non-fluorescent states (Widengren \& Schwille, 2000) with a very complex underlying photophysics (Köhn et al., 2002; Heilemann et al., 2005), which makes it the ideal candidate for checking the robustness of diffusion coefficient measurements based on $2 \mathrm{fFCS}$.

FCS curves were measured using different values of total excitation power per laser between 5 and $40 \mu \mathrm{W}$. A typical measurement result is shown in Fig.3.5 displaying the ACFs for each focus as well as the CCF between photons from different foci for a total excitation power of $7 \mu \mathrm{W}$ per laser. The figure shows also fits of model curves, Eq.2.8, to the measurements. To take into account the Cy5 cis-trans isomerization, an additional exponential term was included into Eq.2.8: a purely diffusional part was multiplied by a time-dependent factor introducing an exponentially decaying component on a microsecond time scale (Widengren, et al., 1994, Widengren \& Schwille, 2000). The lower panel of the figure shows the residuals between fitted and measured curves, demonstrating the fair quality of the fit. 

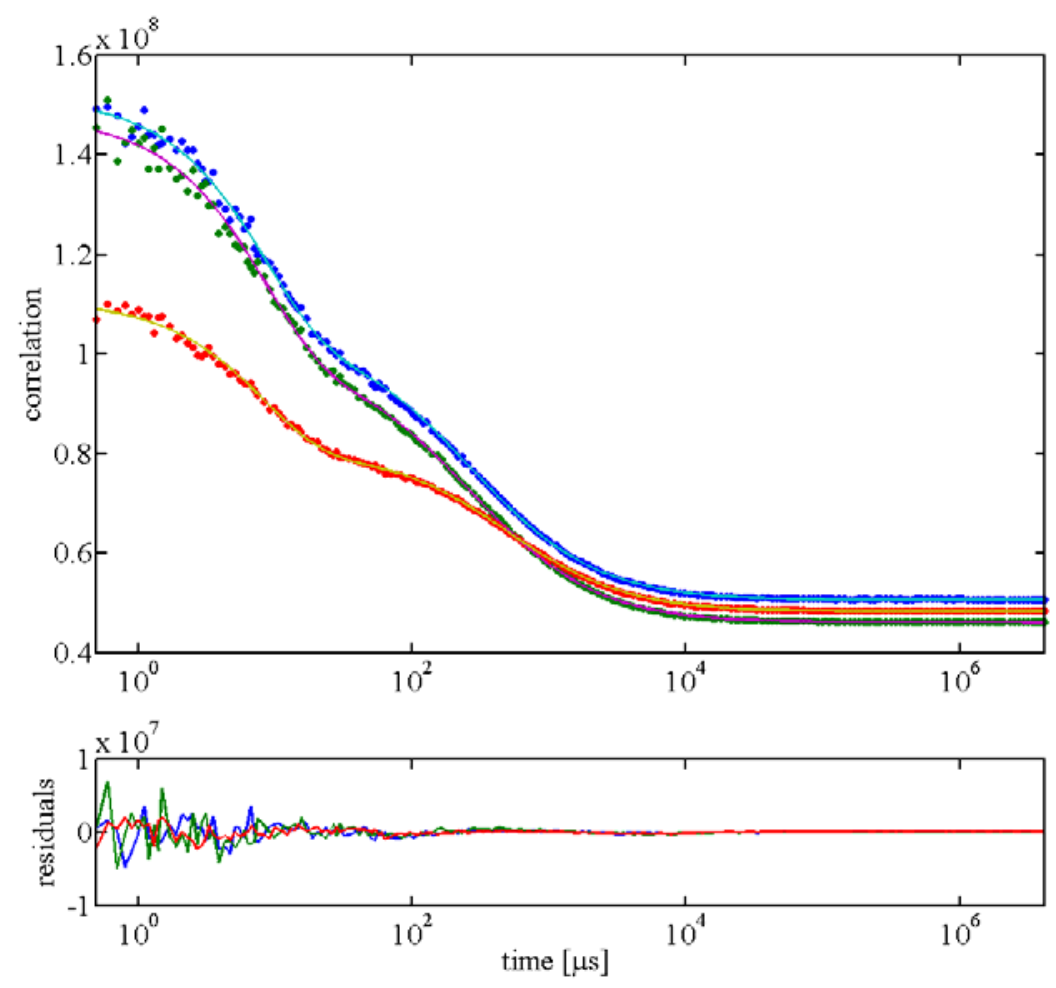

Fig.3.5: Auto- and cross-correlation functions measured for Cy5 with $7 \mu \mathrm{W}$ total laser power per excitation focus. Although both ACF curves have the same shape, their amplitudes are slightly different due to a minute power difference between both lasers.

For comparing 2fFCS with conventional single-focus FCS, FCS measurements were performed with the same experimental system but using only one of both lasers. The resulting ACFs were fitted using the same equations as in the case of $2 \mathrm{fFCS}$, but with $\delta=0$. The diffusion coefficient of Cy5 as determined with $2 \mathrm{fFCS}$ was used as the reference value for calibrating the single-focus FCS results.

The final dependence of the determined values on the diffusion coefficient as a function of total excitation power per focus is depicted in Fig.3.6. As one can see, the values as determined with $2 \mathrm{fFCS}$ are insensitive to the excitation power within the range of employed values, giving an average absolute value of the diffusion coefficient of $D_{25^{\circ} \mathrm{C}}(\mathrm{Cy} 5)=(3.7 \pm 0.15) \cdot 10^{-6} \mathrm{~cm}^{2} / \mathrm{s}$ (this value was derived from the experimental values by recalculating it to a temperature of $25^{\circ} \mathrm{C}$ using the Stokes-Einstein equation and the known temperature dependence of the viscosity of water). In contrast, the values obtained with conventional FCS are strongly dependent on excitation power. For better comparison with 2 fFCS, I extrapolated this dependence toward zero excitation power (dotted line) and 
used the obtained zero-intensity value as reference point for all single-focus FCS measurements as was mentioned in section 3.1.

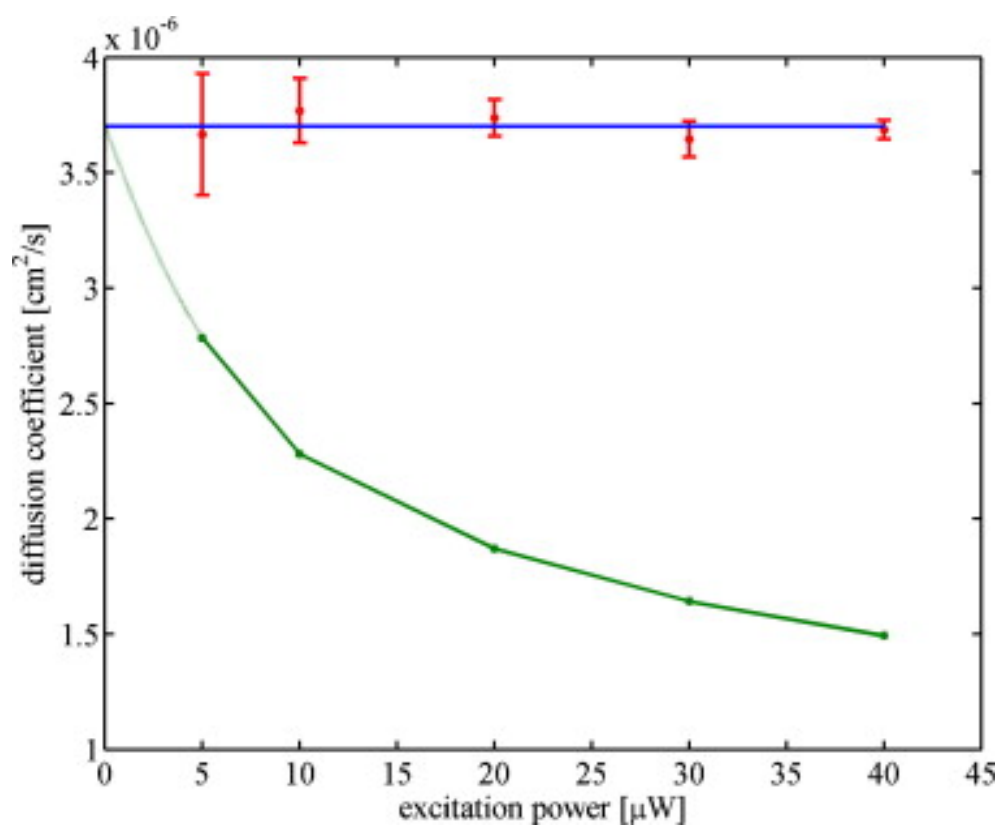

Fig. 3.6: Determined diffusion coefficient as a function of total laser excitation power per focus. Points with error bars are the results of $2 \mathrm{fFCS}$. Solid horizontal line shows the average value of all 2 fFCS measurements. Lower intensity dependent curve refers to the results of conventional FCS, using the extrapolated zero-intensity value as reference. Dotted line is an extrapolation of the determined power dependence toward zero power (Loman et. al., 2008).

\subsection{Summary}

The dye Cy5 exhibits strong optical saturation due to optically driven cis-trans isomerization. It is a very similar process to triplet-state pumping and therefore the obtained results can be interpreted using the theoretical calculations discussed above.

The dependence of the apparent diffusion coefficient of $\mathrm{Cy} 5$ on excitation power, as determined with conventional single-focus FCS, is in perfect qualitative agreement with the theoretical estimates (compare Figs.3.4 and 3.6). Interestingly, the $2 \mathrm{fFCS}$ experimental results do not show the predicted shift towards smaller values within the range of employed excitation power values. It may be due to the fact that the cis-trans isomerization is accelerated by light in both directions. However, 2fFCS measurements of the diffusion of other dyes such as Rhodamine 6G and Oregon Green studied in 
chapter 2.4 show a similar insensitivity upon excitation power within a sufficiently small range of excitation intensities (Müller C.B et al., 2008c).

Nevertheless, the result shown in Fig.3.4 makes clear that as soon as triplet state pumping or similar light-driven photophysical processes take place, it is always advisable to check the dependence of the determined diffusion coefficient on excitation intensity when using $2 \mathrm{fFCS}$ as well as conventional FCS. 



\section{Molecular sizing with dual-focus fluorescence correlation spectroscopy}

This chapter describes the application of dual-focus FCS for size determination of various molecules. Taking into account the results of the previous chapter, all experiments were done using low excitation intensity and relaxed laser focusing (optimal excitation conditions). I start with illustrating the 2 fFCS superb accuracy. Then, I present results for chemically synthesized dyes, and for short oligopeptides. The observed quantitative relation between molecular weight and measured diffusion coefficient is discussed. At the end of the chapter, I present results for several globular proteins.

\subsection{Accuracy of dual-focus fluorescence correlation spectroscopy}

In chapter 2 it was shown that the accuracy of a 2 fFCS measurement is about $1 \%$. In order to show that the method is indeed able to determine diffusion coefficients with high precision, we measured the diffusion coefficient of Atto655 free acid and its two derivatives NHS-ester and maleimide.

Atto655 has the particular property that it has an exceptionally small intersystem crossing rate and therefore negligible triplet state dynamics. That means that the autocorrelation (ACF) and cross-correlation (CCF) functions do not show any fast exponential decay on the microsecond time scale, which is so typical for triple-state dynamics. The correlation curves of Atto655 are thus solely determined by diffusion. However, one should keep in mind that the slight increase of triplet lifetime can be observed when the dye is bound to other molecules, e.g. in an Atto655-DNA complex (Eggeling et al., 2006). Besides that, Atto655 fluorescence is efficiently quenched by the amino acid tryptophan and the DNA base guanine (Doose et al., 2005).

Three forms of Atto655: free acid (AD 655-21), NHS-ester (AD 655-31) and maleimide (AD 655-41) were all purchased from Atto-Tec GmbH (Siegen, Germany) and were used without any further purification. For the 2fFSC measurements the dyes were dissolved in bi-distilled water (Millipore $\mathrm{GmbH}$, Germany) and then diluted to a $0.5-$ $0.2 \mathrm{nM}$ concentration. All experiments were performed at ambient temperature (20$22^{\circ} \mathrm{C}$ ). The temperature of the sample solution was monitored with a digital thermometer HH506RA (Newport Electronics GmbH, Germany). The values of the diffusion coefficients reported here were recalculated to a temperature of $25^{\circ} \mathrm{C}$ for standardization purpose using the Stokes-Einstein equation and knowing the temperature dependence of water's viscosity. The diffusion coefficient measurements for each dye sample were repeated at least 5 times. 


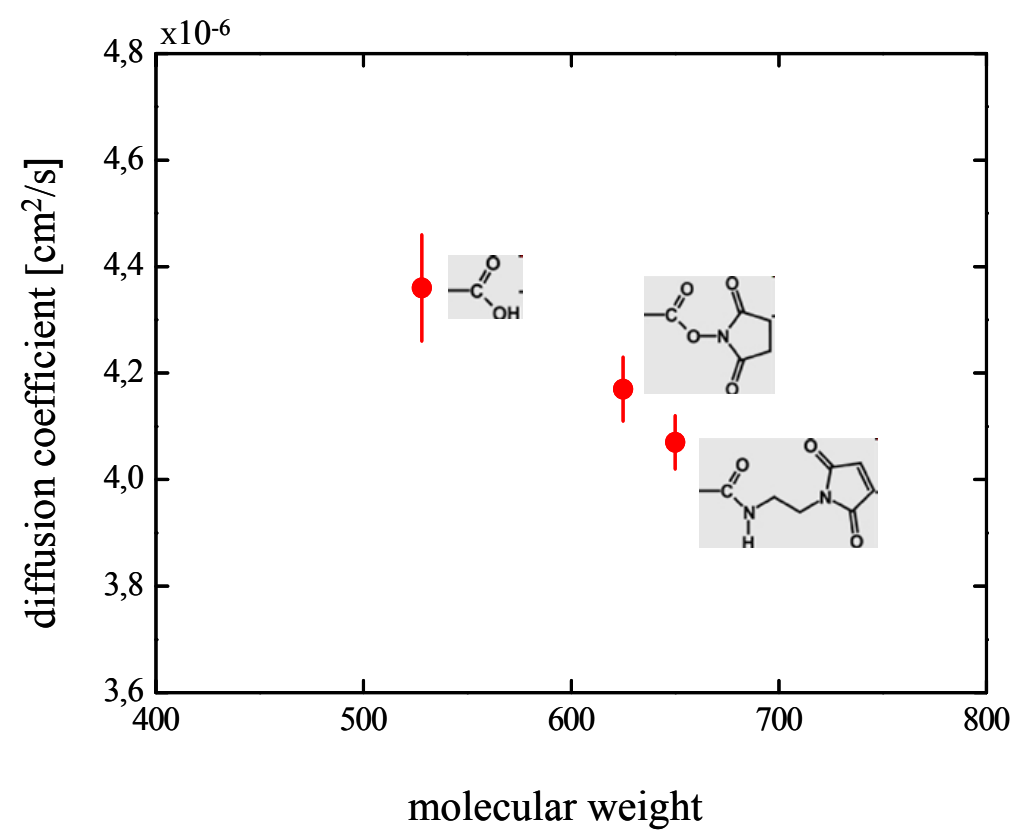

Fig.4.1: The diffusion coefficients of the three forms of Atto655. Red circles with error bars are the results of $2 \mathrm{fFCS}$, using 10 measurements to determine a standard deviation of the diffusion coefficient. The chemical structure of the side groups, i.e. free acid, NHS ester and maleimide (from left to right) are depicted next to the corresponding diffusion coefficient values.

ACFs and CCF of all three forms of Atto655 showed purely diffusion-related correlation decay (Fig.1.6), as expected, and the measured curves were fitted with Eq.2.8 (see chapter 2). The results are depicted in Fig.4.1 and collected in Table 4.1. As can be seen, in spite of the small difference in structure, the diffusion coefficients of all three Atto655 forms could still be resolved in a 2fFCS experiment. Pulsed field gradient NMR or pfgNMR, an independent method capable of measuring absolute diffusion coefficient values (for details see chapter 1), was used for the cross-check measurements. The diffusion coefficient values of Atto655 free-acid and its maleimide derivative were determined in deuterized methanol with pfgNMR. Using those diffusion coefficients, the hydrodynamic radii were calculated via the Stokes-Einstein relation in both solvents. The found values were identical within measurement error. The hydrodynamic radius of Atto655-COOH and Atto655-maleimide were found to be equal to $(5.68 \pm 0.19) \AA$ and $(6.01 \pm 0.11) \AA$, respectively. Thus, $2 \mathrm{fFCS}$ was sensitive enough to be able to resolve side group variations leading to diffusion coefficient differences of only $4 \%$.

What is the minimal molecular weight difference that can be resolved using $2 \mathrm{fFCS}$ ? The diffusion coefficient of a molecule depends on its hydrodynamic radius and thus on the 
cubic root of the molecular weight for spherical particles. This relation restricts the sensitivity of FCS. In our case, the molecular weight of the Atto655-derivatives with NHSester and maleimide side groups is $15 \%$ and $20 \%$ larger than that of Atto655-COOH. This difference translates into respective differences of $3 \%$ and $4 \%$ in the diffusion coefficient, which could still be resolved with 2fFCS (see Fig.4.1 and Table 4.1). But the difference between the diffusion coefficients of the NHS-ester and maleimide forms that corresponded to a $4 \%$ difference in molecular weight was already as small as our measurement error (Fig.4.1). However, the larger mean value of the Atto655-NHS diffusion coefficient was still visible. In conclusion one can say that a molecular weight difference of $15-20 \%$ represents the lower limit which can still be resolved with certainty by $2 \mathrm{fFCS}$.

\subsection{Quantitative relation between molecular weight and the diffusion coefficient}

Intuitively one expects that the more massive a molecule is, the slower it diffuses. This is indeed the case; however, the molecular mobility and the molecular weight do not have a simple relationship. Hence it is difficult to predict hydrodynamic characteristics of molecules in solution, for example their diffusion coefficient or sedimentation constant, when only their molecular weight (MW) is known or vice versa. To have a closer look at this problem, we measured and analyzed the diffusion coefficients of different molecules with known molecular weight.

\subsubsection{Red fluorescent molecules}

Besides the three derivatives of Atto655 (free acid, NHS-ester, and maleimide) as presented in previous section, I measured also the diffusion of Cy5-NHS-ester (PA15101), Cy5-maleimide (PA15131) (GE Healthcare, München, Germany), and Alexa Fluor ${ }^{\circledR}$ 647NHS-ester (A-20006, Invitrogen GmbH, Karlsruhe, Germany). Aqueous solutions of these dyes were prepared in bi-distilled water. The final concentration of the fluorescent molecules was adjusted to lie in the range of $0.2-0.5 \mathrm{nM}$. All the dyes were purchased as powder that contained complexes of the dye with corresponding counter ions. After dissolving the dye was in either cationic or anionic form. To make a reliable comparison of the different diffusion coefficients as a function of MW, the knowledge of the exact MW of the ionic form was required. Therefore a mass spectrometry method termed 'electrospray ionization' was used to determine the MW of Atto655-maleimide as well as Cy5 and Alexa Fluor $^{\circledR} 647$ in NHS-ester form. It was found that the MW of Atto655-maleimide was equal to $648 \mathrm{Da}$ and corresponds to the cationic form of the dye with a MW of $650 \mathrm{Da}$ as provided 
by the company. Therefore the MW values for the other derivatives of Atto655 were taken from the Atto-Tec specification list. Those are equal to $625 \mathrm{Da}$ and $528 \mathrm{Da}$ for the NHSester and the free acid form, respectively. The MWs of Cy5-NHS-ester and Alexa Fluor $^{\circledR} 647$ NHS-ester were found to be equal to $780 \mathrm{Da}$ and $1135 \mathrm{Da}$, respectively. The difference in molecular weight between NHS-ester and maleimide side group is only $25 \mathrm{Da}$ - hence, Cy5-maleimide has a MW of 805 Da.

All 2fFCS measurements were done on the system described in chapter 2. A rather low laser intensity of $7 \mu \mathrm{W}$ was chosen for excitation to avoid any saturation due to Cy 5 cistrans photophysics and the complex photophysics of Alexa Fluor ${ }^{\circledR} 647$ which involves both cis-trans isomerization as well as triplet state formation. To take into account those processes, additional exponential terms were included into Eq.2.8 to describe fast decays in the correlation curves on the microsecond time range. It should be noted that the determined diffusion coefficients of Cy5-NHS-ester and Cy5-maleimide were found to be virtually identical within our measurement error. Therefore, in what follows, the notation "Cy5" and "Alexa Fluor ${ }^{\circledR}$ 647" will be used without further side group specification. The measured diffusion coefficients (and thus hydrodynamic radii) and the MW of all the measured red fluorescent dyes are summarized in Table 4.1 .

Table 4.1: Diffusion coefficient of the red fluorescent dyes

\begin{tabular}{lccc} 
Fluorescent dye & $\mathrm{MW}^{+} / \mathrm{Da}$ & $\mathrm{D}_{25^{\circ} \mathrm{C}} / 10^{-6} \mathrm{~cm}^{2} / \mathrm{s}$ & $\mathrm{R}_{\mathrm{h}} / \AA$ \\
\hline Atto655-COOH & 528 & $4.30 \pm 0.15$ & $5.68 \pm 0.19$ \\
Atto655 NHS ester & 625 & $4.14 \pm 0.10$ & $5.86 \pm 0.14$ \\
Atto655 maleimide & 650 & $4.07 \pm 0.10$ & $6.01 \pm 0.11$ \\
Cy5 & 780 & $3.68 \pm 0.09$ & $6.64 \pm 0.22$ \\
Alexa Fluor ${ }^{\circledR} 647$ & 1130 & $3.25 \pm 0.10$ & $7.52 \pm 0.23$ \\
\multicolumn{2}{r}{ MW of the ionic form of the dyes. } & &
\end{tabular}

\subsubsection{Short peptides}

Next, I measured the diffusion coefficients of oligopeptides with amino-acid sequences going from one to eight amino-acids of the FKPYDAAD sequence. The oligopeptide FKPYDAA is a short peptide that has a cleavage site for digestive proteases 
such as pepsin (Dunn, 2001) and can be used in an assay for detecting the fungus Aspergillus fumigatus. That fungus is widespread in nature and is capable of causing a range of various diseases in immuno-compromised individuals such as organ transplant recipients or patients with AIDS or leukemia.

Short peptides were synthesized and labeled by W. Weinig (Dept. Functional Genomics, German Cancer Research Center, Heidelberg). The shortest molecule contains a dye, a linker and only one amino acid (F), while the longest one had the structure dye-x-xFKPYDAAD-biotin, where $\mathrm{x}-\mathrm{x}$ stands for the linker. MALDI mass spectrometry (MALDIMS) was applied to check the synthesis quality of the oligopeptides as well as their MW. These experiments were done in the German Cancer Research Center in Heidelberg. Alexa Fluor $^{\circledR} 647$ was used to label all peptides, so that their diffusion could be measured with $2 \mathrm{fFCS}$.

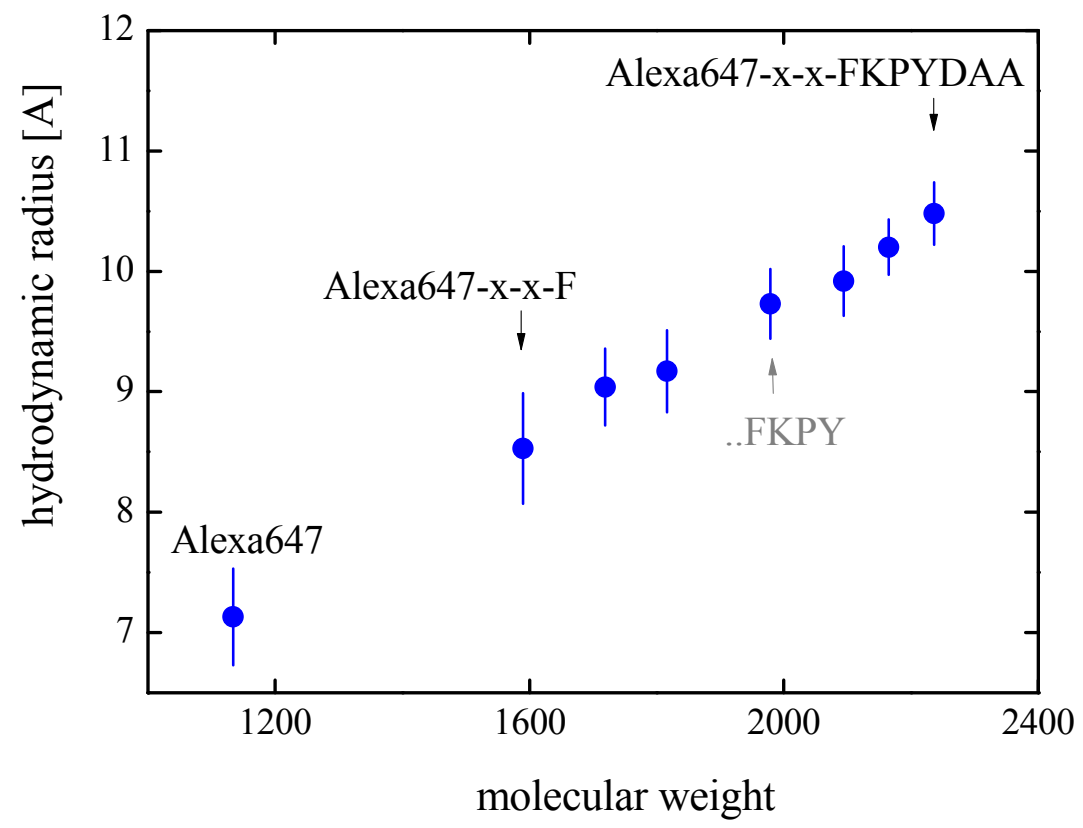

Fig. 4.2: Hydrodynamic radii of Alexa Fluor ${ }^{\circledR} 647$ and seven short peptides coupled with Alexa Fluor $^{\circledR} 647$ via linker. Standard deviation was determined using minimum five measurements for each peptide.

Phosphate buffered saline (PBS) was used to buffer the oligopeptides at $\mathrm{pH}$ 7.4. The diffusion coefficient of Atto655-maleimide was measured in PBS to check the buffer viscosity. It was found that Atto655 maleimide exhibits the same diffusion behavior (within measurement error) in PBS as in bi-distilled water. 2fFCS measurements were done five 
times for each sample. The power of each excitation laser was set to $7 \mu \mathrm{W}$. The determined values of the hydrodynamic radii for all oligopeptides are shown in Fig.4.3. As can be seen, the accuracy of $2 \mathrm{fFCS}$ allowed for distinguishing with certainty between oligopeptides that differed by two amino acids in length only.

\subsubsection{Discussion}

To quantify the relation between the MW and the diffusion coefficient, I employed the so-called Mark-Houwink-Kuhn-Sakurada (MHKS) model. This power-law is usually used in polymer science to get an estimate of the MW or molecular shape (Harding, 1997; Creighton, 1999) of long polymer molecules. The MHKS relation between diffusion coefficient $D$ and molecular weight MW reads:

$$
D=K \cdot \mathrm{MW}^{-\varepsilon}
$$

where $K$ is the MHKS constant (Mark, 2007). Depending on the experimental method, different exponents $\varepsilon$ in the MHKS relation are employed (Harding, 1997). The value of $\varepsilon$ is characteristic for the conformational state of a macromolecule: for example, the value $\varepsilon=0.333$ describes globular macromolecules with spherical shape (Creighton, 1999).<smiles>CCN1CCCc2cc3c(cc21)Oc1cc2c(cc1=N3)CCC[N+]=2CCCC(=O)O</smiles>

MR121 free acid

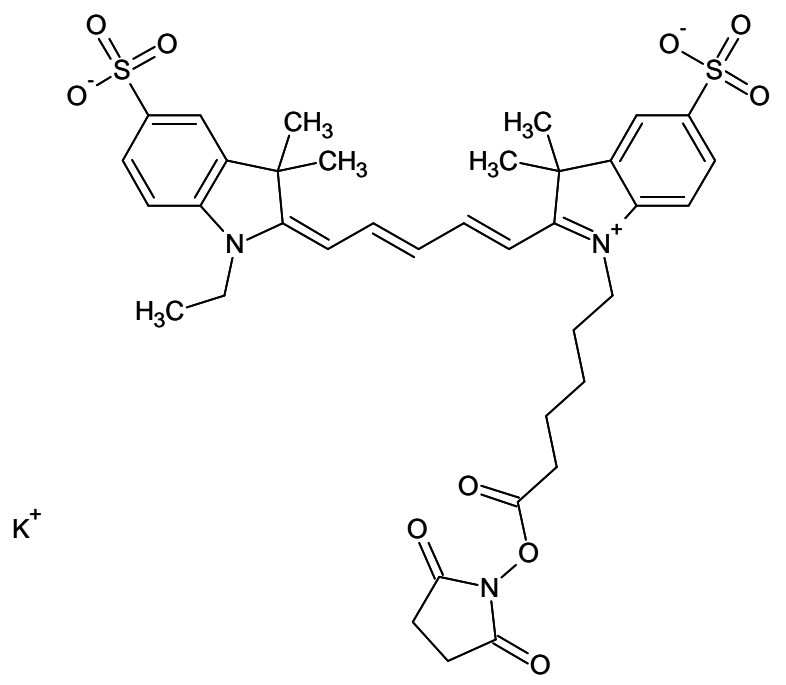

Cy5 NHS ester

Fig. 4.3: Chemical structure of two classes of red fluorescence dyes. MR121 belongs to oxazine molecular group and represents structure similar to Atto655. Both Cy5 and Alexa Fluor ${ }^{\circledR} 647$ are cyanine dyes. Atto655 and Alexa Fluor $^{\circledR} 647$ have additional or different side groups to tune their property, e.g. solubility and photostability, but their structures are a commercial secret. 


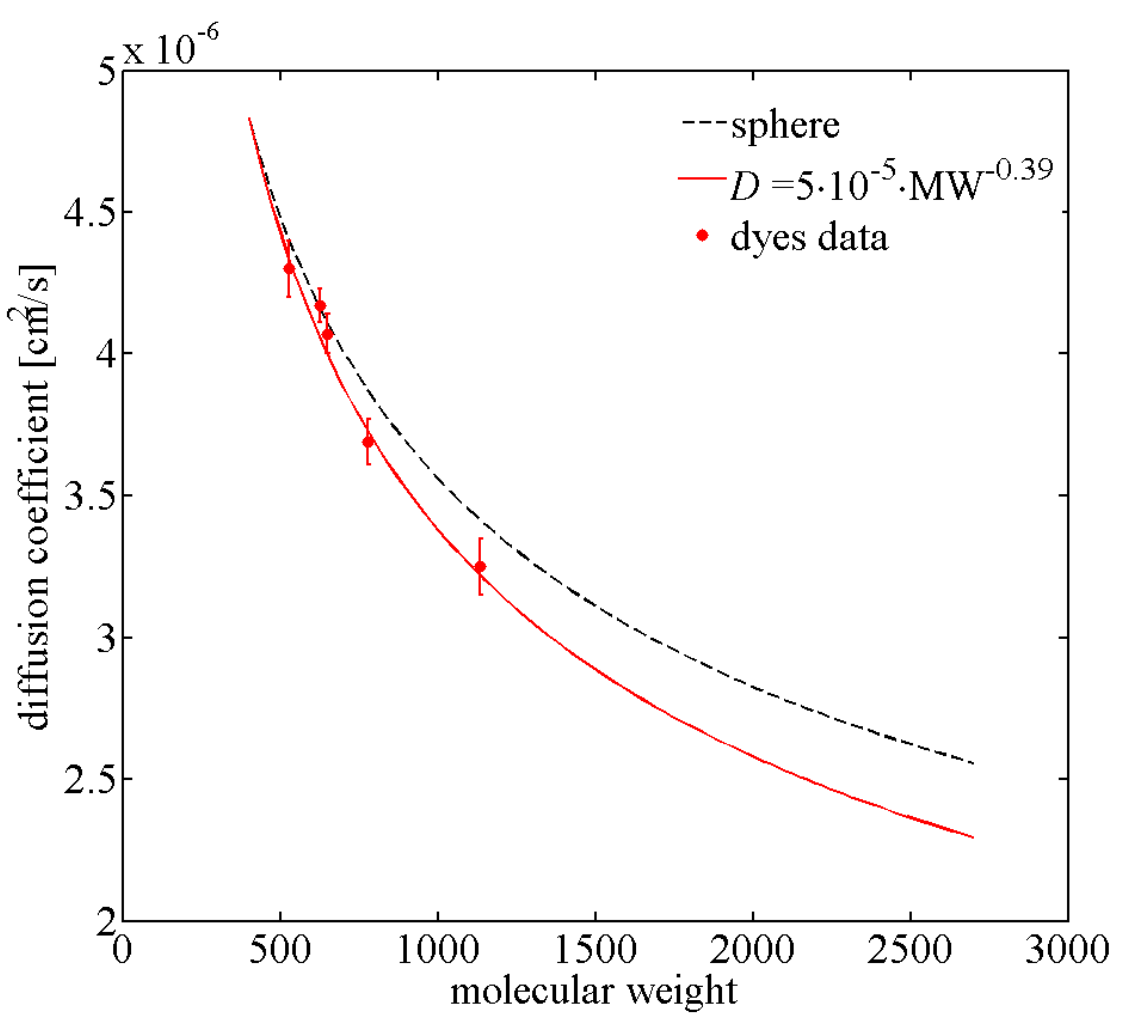

Fig.4.4: Diffusion coefficients of fluorescent dyes' molecules are shown. All values correspond to $25^{\circ} \mathrm{C}$ and were obtained in aqueous solutions. Circles with error bars are the experimental results of $2 \mathrm{fFCS}$, using ten measurements for each point to determine a standard deviation of the diffusion coefficient. The lines of the same color represent Mark-Houwink-Kuhn-Sakurada relations with the fitted constant and exponent for each set of the experimental data. Black dash line represents calculated MKHS relation in case of spherical particles. Constant was chosen in such way that fitted curve and calculated MHKS relation return the same value for molecular weight of $\mathbf{4 0 0}$.

The red fluorescent dyes investigated here are molecules with rather elongated and planar shape (Fig.4.3). However, due to their small size (5 to $8 \AA$ in radius, see Table 4.1 ) and correspondingly fast rotational diffusion in the picoseconds range, one can assume that, on a millisecond timescale, their translational diffusion behavior might be close to that of small spheres. To check that assumption, the MHKS relation with the exponent equal to 0.333 was used to compare the diffusion the fluorescent dyes with that of ideal spheres. The result is plotted in Fig.4.3 (black dashed line). The dependence of the diffusion coefficient on MW of the anionic and cationic dye forms is plotted there as well. Red circles with error bars represent experimental values with standard deviation as calculated from at least ten measurements. The experimental values are close to the ideal sphere curve but do not completely follow it, especially in the cases of Cy5 and Alexa Fluor ${ }^{\circledR}$ 647. To find the MHKS constant and exponent which best describe the dye diffusion, the experimental 
results were fitted with the MHKS relation (Eq.4.1). The resulting curve is depicted as the red solid line together with the final equation in Fig.4.4. The exponent was found to be equal to 0.39 . Thus the experimental values of the smallest dyes are in a good agreement with an ideal sphere assumption, but for bigger dyes this assumption fails. The reason might be that the flexibility of the cyanine dye molecules affect their diffusion and shifts their diffusion coefficients closer to the random coil case which has a characteristic exponent $\varepsilon$ between 0.5 and 0.6 (Creighton, 1997).

Let us next consider the oligopeptides. Those molecules can be considered as unstructured chains of varying length. To put the measured dependence of the diffusion coefficient on MW in relation to the MKHS model, two extreme case of the MHKS relation were considered: that for globular proteins and that for random coil (unstructured) polypeptides.

The globular protein case is the simplest one because it is similar to the case of an ideal sphere. If the density $\rho$ of the protein is known, then one finds the following relation between its MW and its hydrodynamic radius $R$ :

$$
\mathrm{MW}=\rho \cdot N_{A} \cdot \frac{4 \pi}{3} R^{3}
$$

where $N_{A}$ is Avogadro's constant. By using the Stokes-Einstein relation, Eq.4.2 can be rewritten as:

$$
D=\frac{k_{B} T\left(4 \pi \rho \cdot N_{A}\right)^{1 / 3}}{6 \pi \eta(3 \mathrm{MW})^{1 / 3}}=K_{\text {sphere }} \cdot \mathrm{MW}^{-1 / 3}
$$

where $K_{\text {sphere }}$ is a parameter that accounts for protein density, solution temperature, and viscosity. Assuming that the average density of proteins is equal to $1.35 \mathrm{~g} / \mathrm{cm}^{3}$ (a value used in protein crystallography, see Fisher et al., 2004), and using a temperature of $25^{\circ} \mathrm{C}$ and the viscosity of water, $K_{\text {sphere }}$ is equal to $3.68 \cdot 10^{-5} \mathrm{~cm}^{2} / \mathrm{s}$. The corresponding MHKS law is shown as a magenta dashed line in Fig.4.5. There, the experimental values for the diffusion coefficient of the oligopeptides are shown as blue circles. As can be seen, the oligopeptides exhibit a slower diffusion than that for globular proteins of similar MW. 


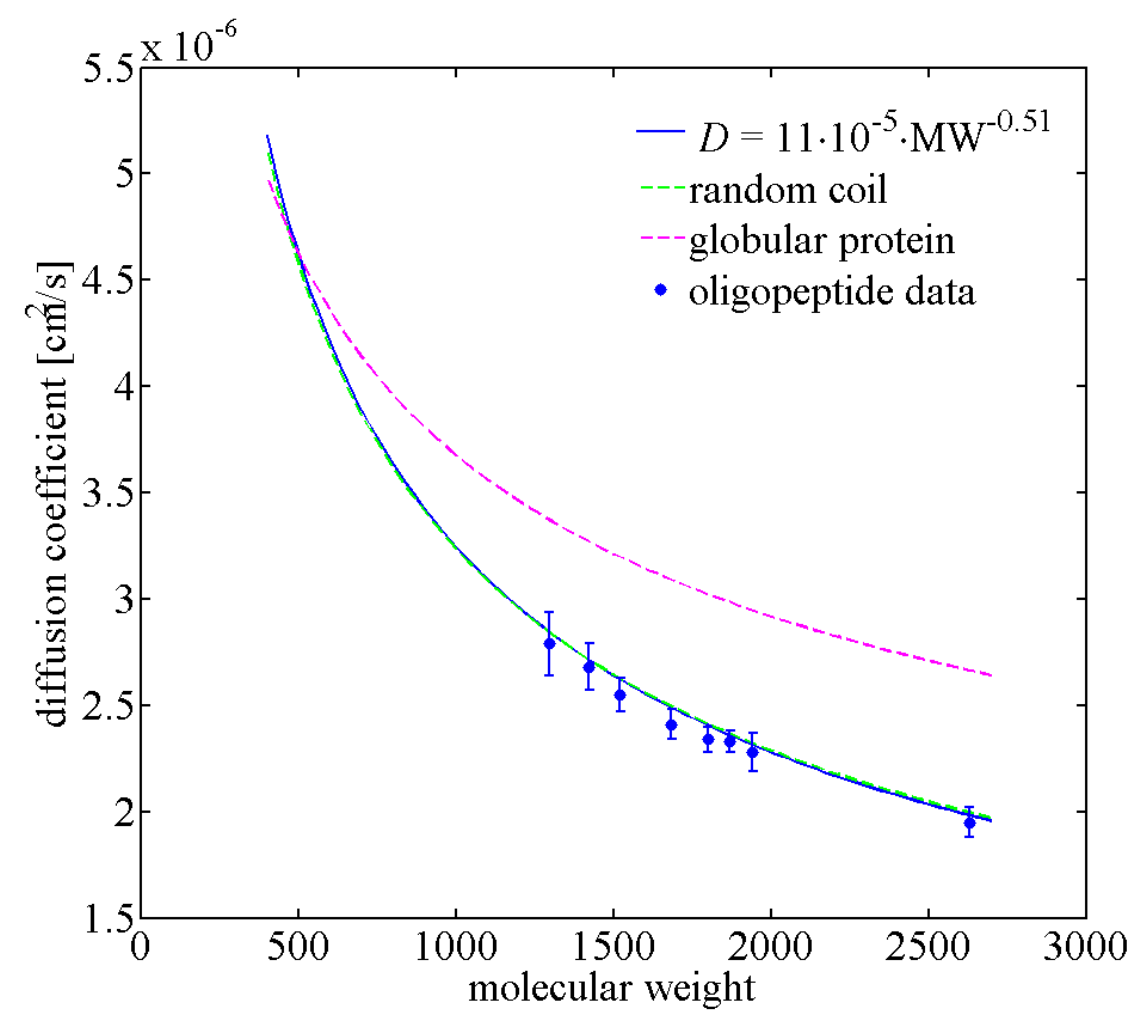

Fig.4.5: Diffusion coefficients of measured oligopeptides. All values correspond to a temperature of $25^{\circ} \mathrm{C}$ and were obtained in PBS buffer at $7.4 \mathrm{pH}$. Circles with error bars are the experimental results from $2 \mathrm{fFCS}$, using ten measurements for each point to determine a standard deviation of the diffusion coefficient. Lines of same color represent Mark-Houwink-Kuhn-Sakurada relations with the MHKS constant and exponent obtaining from fitting the corresponding model to the experimental data. Here, magenta dashed line is the MHKS relation for spherical molecules (globular proteins) with the exponent $\varepsilon$ equal to 1/3 and MHKS constant obtained with the average protein density. Green dashed line represents MKHS relation for a random coil conformation (oligopeptide chains). The corresponding exponent is equal to 0.5 , the MHKS constant was calculated using the average mass of amino acids and a randomchain segment length of $2.79 \AA$.

Let us consider a random coil conformation of an ideal polypeptide chain. Ideal means no interactions between monomers that are far apart along the chain. The simplest unified description of all ideal polymers is provided by an equivalent freely joint chain model. The equivalent chain has the same mean-square end-to-end distance $\left\langle R^{2}\right\rangle$ and the same maximum end-to-end distance $R_{\max }$ as the actual polymer. But it is independent on the local chemical structure of the actual polymer and has $N$ freely-jointed effective bonds or segments of length $b_{0}$ (Kuhn length) (Rubinshtein \& Colby, 2003). The mean-square end-toend distance of this equivalent chain is 


$$
\left\langle R^{2}\right\rangle=N b_{0}^{2}
$$

Taking into account that the MW of a polymer equals the MW of one monomer $M_{0}$ times the number of monomer units (segments) $N$, the expression for the diffusion coefficient of a polypeptide as modeled by the freely-jointed chain model can be written as:

$$
D=\frac{k_{b} T \sqrt{M_{0}}}{6 \pi \eta b_{0}} M W^{-1 / 2}=K_{\text {coil }} \cdot M W^{-1 / 2}
$$

where $K_{\text {coil }}$ is a parameter that includes both solvent characteristics ( $T$ and $\eta$ ) as well as intrinsic parameters of the polymer $\left(M_{0}\right.$ and $\left.b_{0}\right)$. To find a correlation between the diffusion coefficient and the MW of a random coil, $M_{0}$ and $b_{0}$ have to be estimated. The polymer considered here is an oligopeptide with up to 10 amino acids in length. To find a value for $b_{0}$, I used pfgNMR measurement results of the self-diffusion of simple peptides (one to seven amino acids in length) (Danielsson et.al., 2002; Pei et al., 2009). A plot of their hydrodynamic radius as a function of amino acid number is presented in Fig.4.6, together with a fit using Eq.4.4 and choosing $b_{0}$ as the free fit parameter.

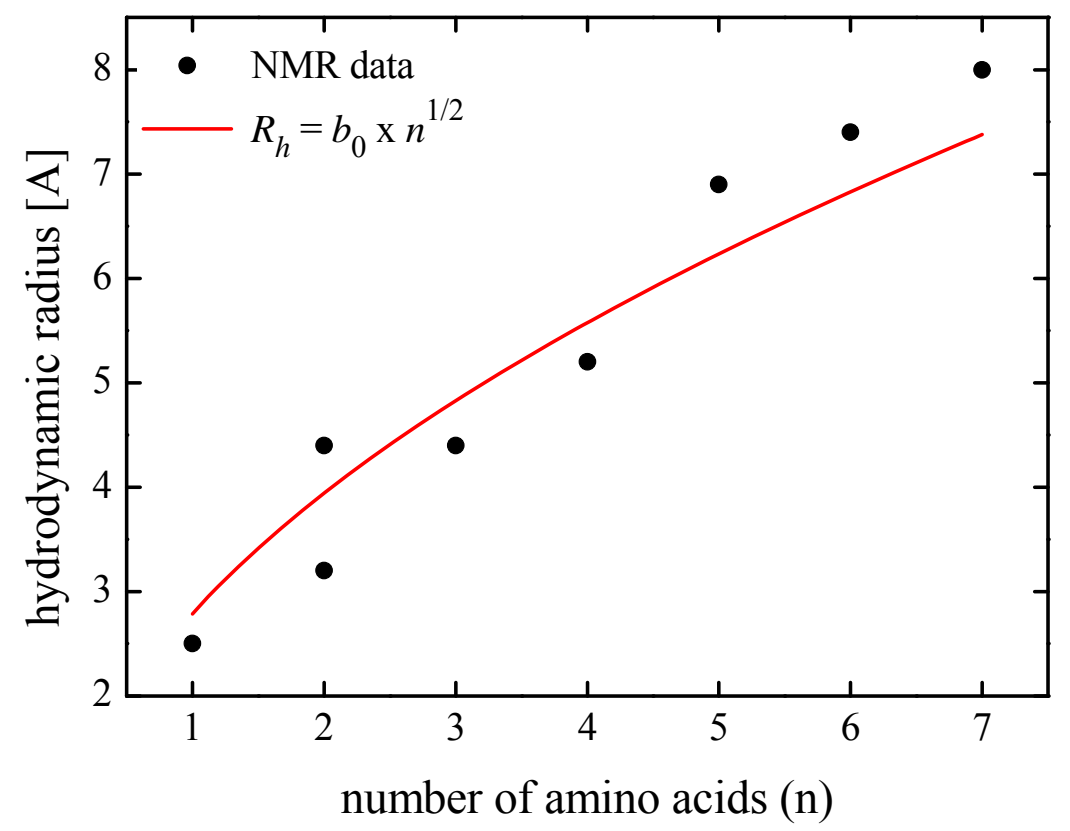

Fig.4.6: Hydrodynamic radius values of short peptides as taken from the literature (Danielsson et al., 2002; Pei et al., 2009) are plotted against the number of amino acids. The Eq.4.6 with was used to fit data and to obtain a value for the segment length $\left(b_{0}\right)$ for the freely joint chain model. 
The estimated Kuhn length was found to be equal $2.79 \AA$. This value is comparable to the literature value of $3.3 \AA$, which is the length of one amino acid (Bier, 2008). Therefore, $M_{0}$ is estimated by using the average molecular weight of an amino acid. $M_{0}$ thus equals to $131 \mathrm{Da}$. The green dashed line in Fig.4.5 corresponds to the resulting MHKS relation for the ideal freely-jointed chain. The fit of the experimental data yields a value for the MHKS exponent of 0.51 (blue solid line in Fig.4.5). Within experimental error, this value is close to the theoretical value of a random coil.

In conclusion, the diffusion behavior of small molecules is not only determined by their molecular weight but also by their shape and flexibility. This is nicely illustrated by the diffusion behavior of fluorescent dyes. The diffusion coefficients for the different derivatives of Atto655 are in a good agreement with ideal sphere assumption, whereas those for Cy5 and Alexa Fluor ${ }^{\circledR} 647$ are not. These last two dyes have a structure that consists of two aromatic rings connected by a conjugated backbone of six methine groups $(\mathrm{CH})$ (Fig.4.4). The polymethine chain makes both dyes much more flexible than Atto655. Therefore, cyanine dye molecules exhibit a slower diffusion coefficient than that of a sphere of the same molecular weight, and their diffusion coefficient lies between the limiting values for a sphere and a random coil. When a cyanine molecule is bound, via a flexible linker, to only one amino acid, its diffusion behaviour is already well approximated by a random coil model (Fig.4.5, first upper point).

Finally, the diffusion behaviour of short peptides labelled with Alexa Fluor ${ }^{\circledR} 647$ via a short linker can be well described by a freely jointed chain model with a segment length of $2.79 \AA$ A. These molecules show flexibility comparable to that of an ideal chain. This is demonstrated in Fig.4.5 by the overlap between the blue solid (fit of the experimental data) and green dashed lines (the model).

\subsection{Globular protein sizing}

In this section, I describe the application of $2 \mathrm{fFCS}$ to the sizing of several common globular proteins (bovine serum albumin or BSA, human serum albumin or HSA, bacterial $\alpha$-amylase, and others). The experimental results are compared with literature values and theoretical calculations based on their crystal structure, as far as that is known.

All proteins are unspecifically labelled with Alexa Fluor ${ }^{\circledR} 647$ or Atto647N. Phosphate buffer saline or PBS and Mops buffers are used to buffer protein solutions at $\mathrm{pH}$ 7.4. The diffusion coefficient of Atto655 maleimide is measured in both buffer systems. The obtained values are identical to the diffusion coefficient value of Atto655 maleimide in 
water (within experimental error), i.e. viscosities of the both buffers are similar to that of water. The experimental data were fitted using Eq.2.8, including additional exponential terms for taking into account cis-trans isomerization and/or triplet state photophysics on the microsecond time scale.

Keeping in mind that optical saturation due to triplet state pumping can affect measurement results (section 3.5.1), and that dye-protein interactions can alter a dye's photophysics, control experiments had to be performed which made sure that all these effect do not bias the determination of the diffusion coefficients. For example, $\alpha$-amylase was labeled with either Alexa Fluor ${ }^{\circledR} 647$ or Atto647N. Both dyes exhibit significantly different photophysical behavior: Atto647N shows very prominent triplet state pumping (intersystem crossing), while Alexa Fluor ${ }^{\circledR} 647$ has both triplet state and cis-trans dynamics. It was found that for both dyes the resulting values of the hydrodynamic radius (as calculated via the Stokes-Einstein relation) are the same within experimental error. However, when a similar control experiment was done for BSA, the hydrodynamic radius of BSA-Atto647N was found to be by $8 \%$ larger than that of BSA-Alexa Fluor ${ }^{\circledR} 647$. A possible explanation is that the strong interaction between BSA and the dyes induces a conformational change in the protein. BSA possesses a special binding pocket for transporting fatty acids, and Atto647N is known to be rather hydrophobic. Thus, it can be assumed that Atto647N preferentially attaches to that binding pocket and induces a change of BSA size and shape.

Interactions between Alexa Fluor ${ }^{\circledR} 647$ and BSA cannot be excluded as well. The experimental data obtained from BSA-Alexa Fluor ${ }^{\circledR} 647$ can be fairly well fitted with only one additional exponential term in Eq.2.8 which means that either the triplet state pumping or cis-trans isomerization is suppressed due to the BSA-dye interaction. In contrast, the fluorescence correlation of both $\alpha$-amylase-Alexa Fluor ${ }^{\circledR} 647$ as well as HSA-Alexa Fluor ${ }^{\circledR}$ 647 show both triplet state pumping as well cis-trans-isomeraization, although BSA is by about $76 \%$ similar to HSA (Huang et al., 2004), and both BSA and HSA fulfil similar functions in different organisms. This demonstrates that it is always advisable to prepare several samples where a protein is labelled with different dyes, so that any dye-specific bias of the diffusion coefficient determination can be checked.

Protein labeling is done in such way that, on average, only one dye or none is attached to a protein. This assures that the dye labeling does not introduce a significant increase in the overall size of a protein. This can be easily seen when recalling that most globular proteins can be well approximated by a spherical geometry, so that the relation $D \propto r_{s}^{-1} \propto V_{s}^{-1 / 3} \propto M W^{-1 / 3}$ holds (combination of Eqs. 4.3 and 4.4). Then, the impact of the 
attached dye on the size of the protein-dye complex can be estimated by calculating the ratio of the MW of the pure protein and the protein-dye complex. For example, the molecular weight of BSA is $66 \mathrm{kDa}$ and that of Alexa Fluor ${ }^{\circledR} 647$ and Atto647N NHS-esters are $1.1 \mathrm{kDa}$ and $0.7 \mathrm{kDa}$, respectively. Thus, I find

$$
\frac{D^{\prime}}{D}=\left(\frac{66+1.1}{66}\right)^{1 / 3} \cong 1.006
$$

which predicts a relative change in the diffusion coefficient upon dye labeling of about $0.6 \%$ for Alexa Fluor ${ }^{\circledR}$ 647, and similarly of $0.4 \%$ for Atto647N. For smaller proteins such as $\alpha-$ amylase, that number will be higher but never more than $1 \%$.

I will start with reporting the results for BSA and HSA. Both proteins belong to the class of serum albumins, which are the most abundant plasma proteins in humans and other mammals. BSA is the best studied example of a serum albumin and has attracted considerable attention over the past decades (see Tabak et al., (2006) and references therein). Similarly, HSA is in the focus of biochemical and biophysical research up to this day (see Otosu et al. (2010)). Fig.4.7 shows typical 2fFCS measurement results for the diffusion of HSA and BSA. The hydrodynamic radius of HSA is found to be equal to $(33.4 \pm 1.8) \AA$, whereas the hydrodynamic radius of BSA is found to lie in the range from $(33.9 \pm 1.6) \AA$ to $(36.8 \pm 2.0) \AA$, depending on which dye was used (Alexa Fluor ${ }^{\circledR} 647$ or Atto647N). The literature values of for BSA range also between $3.4 \mathrm{~nm}$ and $3.7 \mathrm{~nm}$ (Flecha et al., 2003; Murtaza et al., 1999; Ferrer et al., 2001).
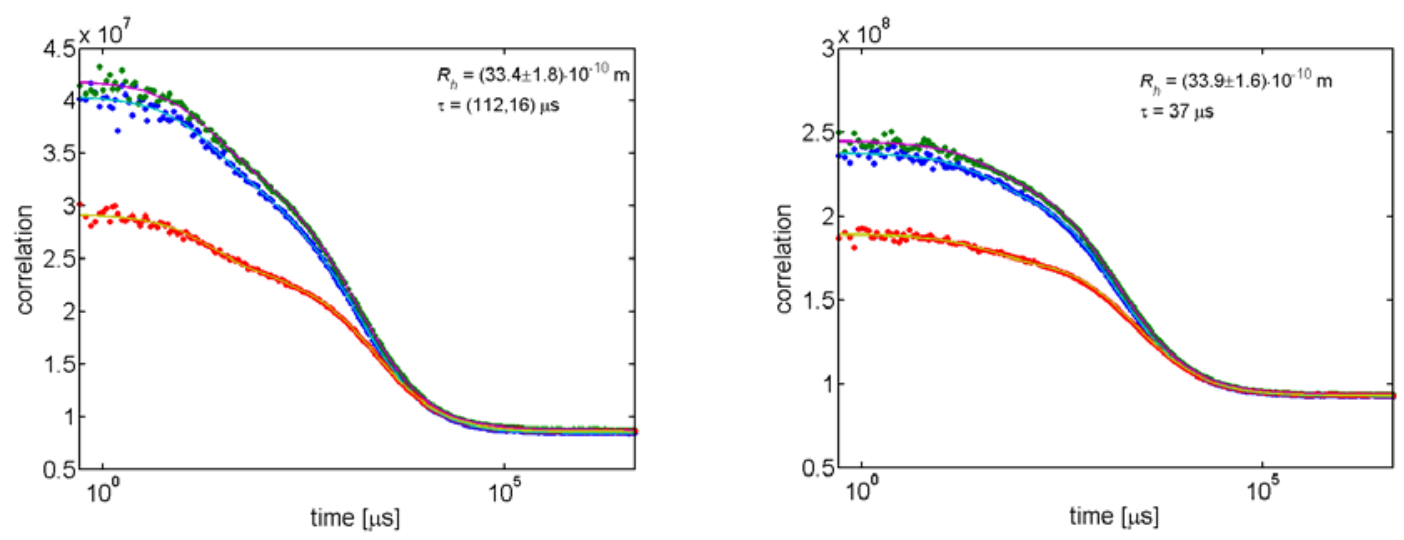

Fig 4.7: Auto- and cross-correlation functions measured for BSA (left panel) and HSA (right panel). Here, both proteins are labeled with Alexa Fluor ${ }^{\circledR} 647$. 
Next, I studied conalbumin and hen egg ovalbumin. These proteins were purchased from GE Healthcare (München, Germany) as a part of a gel filtration calibration kit. The hydrodynamic radius of ovalbumin and conalbumin are known to be $30.5 \AA$ and $35.0 \AA$, respectively (Wishnia et al., 1961; Sabaté \& Estelrich, 2001). 2fFCS measurements yielded values of $R_{h}($ ovalbumin $)=(32.2 \pm 1.6) \AA$ and $R_{h}($ conalbumin $)=(35.8 \pm 1.6) \AA$. Another protein that is used for calibration is rabbit muscle aldolase (GE Healthcare, München, Germany)). Aldolase has a hydrodynamic radius of $48.1 \AA$ according to company specifications. The value obtained by $2 \mathrm{fFCS}$ is $(43.5 \pm 1.7) \AA$.

Phosphoglycerate kinase (PGK) is a glycolytic enzyme that catalyzes the conversion of 1,3-diphosphoglycerate to 3-phosphoglycerate generating one molecule of ATP. This kinase has been extensively studied since the seventies of the last century. Nowadays, this well-described protein is used as a model system: for example, a recently reported method for monitoring fast protein dynamics in vivo has been exemplified on PGK (Ebbinghaus et al., 2010). The PGK hydrodynamic radius is found to be $(30.8 \pm 1.5) \AA$, virtually coinciding with the reported values of 30.0 and $30.9 \AA$ (Spragg et al., 1976; Damaschun et al., 1993).

Amylase is an enzyme that breaks starch down into sugar. Bacterial $\alpha$-amylase is a particular interesting enzyme because of its thermostability and ability to thermal adaptation. The reported value of its hydrodynamic radius is $32 \AA$ (Fitter \& HaberPohlmeier, 2004). 2fFCS measurements find the same value, $R_{h}($ amylase $)=(31.8 \pm 0.7) \AA$.

For comparing my measured values with theoretical predictions based on structural data, I performed calculations using the program HydroPro 7c (de la Torre et al., 2000). This program computes the hydrodynamic properties of rigid macromolecules (globular proteins, small nucleic acids, etc) from their atomic-level structure as specified by the atomic coordinates taken from a PDB file. The following table summarizes all the experimenta values of hydrodynamic radius as found with $2 \mathrm{fFCS}$ and compares them with literature values (as far as they were available) and the theoretical predictions. 
Table 4.2: Hydrodynamic radius values of proteins

\begin{tabular}{lc|c|cc}
\multicolumn{1}{c}{ Protein } & \multicolumn{3}{c}{$R_{h} / \AA$} & $\mathrm{MW} / \mathrm{kDa}$ \\
\hline 2fFCS & Literature & $\begin{array}{c}\text { HydroPro 7c } \\
\text { (PDB code) }\end{array}$ \\
\hline BSA & $\begin{array}{l}33.9 \pm 1.6 \\
36.8 \pm 2.0\end{array}$ & $34,35,37$ & -- & 66.0 \\
\hline HSA & $33.4 \pm 1.8$ & -- & $35.5(1 \mathrm{bm} 0)$ & 69.4 \\
\hline Ovalbumin & $31.4 \pm 1.6$ & 30.5 & $30.4(1 \mathrm{vac})$ & 44.2 \\
\hline Conalbumin & $35.8 \pm 1.6$ & 35.0 & $37.7(1 \mathrm{aiv})$ & 75.8 \\
\hline Phosphoglycerate kinase & $30.8 \pm 1.5$ & $30.0(30.9)$ & $30.9(3 \mathrm{pgk})$ & 44.7 \\
\hline$\alpha$-Amylase & $31.8 \pm 0.7$ & 32.0 & 30.7 (1bli) & 55.2 \\
\hline Aldolase & $43.5 \pm 1.7$ & $48.1 *$ & $47.2(1 \mathrm{zah})$ & 156.8 \\
\hline
\end{tabular}

*High molecular weight gel filtration calibration kit, GE Healthcare 


\subsection{Materials and methods}

Chemicals. $\mathrm{NaHCO}_{3}$, phosphate buffered saline (PBS), Mops buffer, DMSO were purchased from Sigma, Fluka (St. Gallen, Switzerland).

Proteins: Bovine serum albumin (A7906), human serum albumin (A3782) phosphoglycerate kinase (P7634) and $\alpha$-amylase (A4551) were purchased from Sigma (St. Gallen, Switzerland). Gel filtration calibration kit was purchased from GE Healthcare (München, Germany) and included the following proteins: hen egg ovalbumin, rabbit muscle aldolase, conalbumin. All proteins were used without any further purification.

Protein labeling. Amine reactive dyes (NHS-ester) were used for nonspecific labelling of lysins on a protein's surface. All dyes were dissolved in dehydrated DMSO to avoid NHS-ester group hydrolysis. Used dye concentration in DMSO was adjusted to 4-8 $\mathrm{mM}$ in order to get a negligible amount of DMSO in the protein solution later on. Aliquots of dye-DMSO solution were frozen and kept at $-20{ }^{\circ} \mathrm{C}$.

All proteins were labeled with either one of the following dyes: Alexa Fluor ${ }^{\circledR}$ 647NHS-ester, Atto647N-NHS-ester or Cy5 bis-NHS-ester, using the standard labeling procedure as described by Invitrogen. Solutions of about $10 \mu \mathrm{M}$ protein concentration were prepared in $0.1 \mathrm{M} \mathrm{NaHCO}_{3}$ (pH 8.3). Alexa Fluor ${ }^{\circledR}$ 647, for example, in DMSO was added in small amount to $1 \mathrm{ml}$ of protein solution to get a protein-dye ratio of 1 to 0.6 . Labeling was performed by incubating a dye with a protein at room temperature for 1-2 hours in the case of BSA and HSA, and overnight for other proteins. Labeled proteins were purified using either PD-10 desalting columns (GE Healthcare Europe GmbH, Freiburg, Germany) or a HPLC system (Jasco Labor und Datentechnik GmbH, Groß-Umstadt, Germany). After elution, the purified proteins were kept in phosphate buffer saline (PBS) at $\mathrm{pH} 7.4$, and were used for the measurements directly after preparation..

All 2fFCS measurements of peptides and proteins were done in Lab-Tek II chambers with a glass cover slide at their base (Nalge Nunc International Corp., Naperville, IL, USA). To prevent unspecific adhesion of peptides or proteins to the glass-surface, the chambers were incubated overnight with a $3 \%$ BSA solution. BSA is a standard surface blocking reagent because of its lack of reactivity in many biochemical reactions (Ishikawa et al., 1987). 


\section{Rotational diffusion and protein sizing}

Using dual-focus FCS one can measure a translational diffusion coefficient and therefore the molecular size with a precision better than $4 \%$. This means that the lower threshold of $2 \mathrm{fFCS}$ sensitivity is about $15-20 \%$ difference of molecular weight as it was illustrated in section 4.1. The reason of this restriction lies in the fundamental relation between the diffusion coefficient and molecule's hydrodynamic radius as given by the Stocks-Einstein equation. However there is another fundamental dynamic process of molecules within solution: thermally induced rotational diffusion. The rotational diffusion coefficient $D_{\text {rot }}$ of a molecule is connected to the value of its hydrodynamic radius $R_{\text {rot }}$ as given by the Stokes-Einstein-Debye equation (Debye, 1929):

$$
D_{r o t}=\frac{k_{B} T}{8 \pi v R_{r o t}^{3}}
$$

The classical methods to determine rotational diffusion constants are static or dynamic fluorescence anisotropy measurements (Lakowicz, 1999). These measurements require fluorescent labels with a fluorescence lifetime in the order of the rotational diffusion time. Taking into account that the rotational diffusion times of macromolecules such as globular proteins are on the order of several dozen nanoseconds, the anisotropy measurements require the use of rather uncommon long-lifetime probes.

In this chapter I present a method to measure rotational diffusion of large biomolecules in solution. The method is based on fluorescence correlation on the nanosecond time scale (Loman et al., 2010). The idea to use fluorescence correlation spectroscopy in the nanosecond time-range to obtain information about molecular rotational motion is nearly as old as the FCS concept (Ehrenberg \& Rigler, 1974; Aragón \& Pecora, 1975). However, until present there are only a few experimental studies to determine rotational diffusion coefficients using FCS (Kask et al., 1987; Tsay et al., 2006; Felekyan et $a l ., 2005)$. Nonetheless, this method is very attractive because it is independent on the label's fluorescence lifetime. Besides, it is also rather independent of the details of excitation and detection polarization. These advantages are winning in contrast to anisotropy measurements (Lakowicz, 1999), especially when large biomolecules are studied.

The conventional approach to measure rotational diffusion using FCS is to excite the sample with a linearly polarized continuous-wave excitation laser, and to monitor the fluorescence through either linear polarizers, or in non-polarized detection mode. Here, I 
propose a more complex but also more efficient excitation/detection scheme: fluorescence is excited by a train of laser pulses with alternating polarization. Fluorescence detection is done by time-correlated single photon counting in two detection channels with crossed detection polarization. High-speed electronics times the photon detection events with picosecond accuracy. By this one can unequivocally assign each detected photon to its exciting laser pulse. Thus, information about excitation and detection polarizations of each photon is obtained. This allows calculating all cross correlations down to picosecond correlation times, making it possible to pick out a correlation function that has largest rotational-diffusion related amplitude. In the next section I will derive the theoretical shape of the various correlation functions that can be obtained. Afterwards the method is exemplified by sizing a set of common globular proteins, using the determined rotational diffusion coefficient.

\subsection{Theory}

\subsubsection{Autocorrelation function (or ACF)}

On a pico- to nanosecond timescale, the ACF is characterized by fluorescence antibunching and rotational diffusion. Fluorescence antibunching is caused by the fact that a single emitter with a finite lifetime of its excited state can just emit one single photon at a time. Rotational diffusion will be seen in the ACF if one excites/detects fluorescence in a polarization-sensitive manner. Due to the rotation of a molecule between different photon excitation and emission events and thus rotation of the molecule's dipole axis into or out of the polarization plane of the detector, the correlation of the recorded fluorescence signal will show a temporal component that is related to the rotational diffusion of the molecule (see, for example, Ehrenberg \& Rigler, 1974).

Let us consider an experiment where the sample is excited with two consecutive pulses of negligible pulse width. If the fluorescence decay is mono-exponential with decay time $\tau$, and a molecule can emit, after one excitation pulse, only one photon, the probability to detect two photons from one and the same molecule with the lag time $t$ between them will be proportional to

$$
\int_{\min (\delta-t, 0)}^{\delta} d t_{1} \frac{\kappa_{1}}{\tau} e^{-t_{1} / \tau} \frac{\kappa_{2}}{\tau} e^{-\left(t_{1}+t-\delta\right) / \tau}=\kappa_{1} \kappa_{2} F_{1}(t, \tau, \delta)
$$


where $F_{1}(t, \tau, \delta)$ is the function

$$
F_{1}(t, \tau, \delta)=\left\{\begin{array}{l}
\sinh (t / \tau) e^{-\delta / \tau}, t \leq \delta \\
\sinh (\delta / \tau) e^{-t / \tau}, t>\delta
\end{array}\right.
$$

$\delta$ is the time delay between the two pulses, and $\kappa_{1}$ and $\kappa_{2}$ quantify the chance that the first and the second pulse lead to a photon detection event, respectively. Eq.5.2 can be understood as the product of the probabilities: (i) that the molecule is excited at time zero, (ii) that it emits a photon at time $t_{1}$, (iii) that the molecule is re-excited by a second pulse at time $\delta$, and (iv) that it emits a second photon at time $t_{1}+t$. Generally, the values of $\kappa_{1}$ and $\kappa_{2}$ depend on the excitation pulse and detection polarization as well as the orientation of a molecule's excitation dipole. For a temporal distance between the two pulses that is much larger than the fluorescence decay time, $\delta>>\tau$, and for lag time values much larger than the fluorescence decay time, $t>>\tau$, this function approaches the simple form

$$
F_{1}(t, \tau, \delta) \rightarrow \frac{1}{\tau} \exp \left(-\frac{|t-\delta|}{\tau}\right)
$$

The chance to detect two photons with lag time $t$ from two different molecules is similar to Eq.5.4, but with the distinction that the upper integration limit is now extended to infinity, leading to

$$
\int_{\min (\delta-t, 0)}^{\infty} d t_{1} \frac{\kappa_{1}}{\tau} e^{-t_{1} / \tau} \frac{\kappa_{2}}{\tau} e^{-\left(t_{1}+t-\delta\right) / \tau}=\kappa_{1} \kappa_{2}\left[F_{1}(t, \tau, \delta)+\frac{1}{2 \tau} \exp \left(-\frac{t+\delta}{\tau}\right)\right]=\kappa_{1} \kappa_{2} F_{2}(t, \tau, \delta)
$$

Eq.5.5 is also the defining equation for $F_{2}(t, \tau, \sigma)$.

The value of $F_{1}(t, \tau, \sigma)$, in contrast to that of $F_{2}(t, \tau, \sigma)$, tends to zero when the pulse delay goes to zero, which is the essence of fluorescence antibunching, reflecting the fact that a single molecule cannot emit more than a single photon per excitation. However, the function $F_{1}(t, \tau, \sigma)$ does not take into account the rotational diffusion of the molecule (i.e. the rotation of its absorption/emission dipole). These contributions are contained in the prefactors $\kappa_{1}$ and $\kappa_{2}$ in Eq.5.2 and will be considered in the next section. 


\subsubsection{Excitation and detection}

To get information about molecular rotational diffusion, the fluorescence excitation and detection conditions of the measurement have to be specified, in particular the polarization properties. Let us assume that the fluorescence lifetime is considerably shorter than the rotational diffusion time, which is mostly the case when studying rotational diffusion of large proteins and using short-lifetime dyes. In what follows I only consider conditions where the protein is tagged with a dye molecule in such a way that the relative orientation of the dye with respect to the protein's principal axes is random but fixed (corotation of dye with protein). Then, one only needs to consider the molecule detection function (MDF) describing the chance to excite and detect a photon for a dye molecule with a given orientation and position in sample space. The calculation of this function can be done using a wave-optics approach as described in ref. (Enderlein et al., 2005).

The MDF, of course, depends on the peculiarities of the excitation, and can be different for different excitation pulses (for example, when exciting the sample with a pulse train of pulses with alternating polarization). A first laser pulse with corresponding MDF $U_{1}(\omega, \vec{r})$ thus 'prepares' the sample in such a way that $U_{1}(\omega, \vec{r})$ describes the chance to detect a photon from an excitation/emission dipole at position $\vec{r}$ having orientation $\omega$. Here the variables $\vec{r}$ and $\omega$ are related to the lab space. However, the movement of the molecule has to be described in the frame of the molecules principle axis (see appendix). Therefore, rotating the distribution $U_{1}(\omega, \vec{r})$ into the protein's frame of principal axes which has orientation $\Omega^{\prime}$ with respect to the lab frame gives the average chance to excite and detect a photon from the protein-dye complex. Next, Green's function $G\left(\Omega, \Omega^{\prime}, t\right)$, gives the chance that the protein-dye complex rotates from orientation $\Omega^{\prime}$ into orientation $\Omega$ within time $t$. Using a similar argument as before, the chance to excite and detect a photon by a second laser pulse with the MDF $U_{2}(\omega, \vec{r})$ is given by a back-rotation of $U_{2}(\omega, \vec{r})$ into the protein's frame.

Finally, by integrating over all possible positions and orientations, one obtains the average of the product $\kappa_{1} \kappa_{2}$ (averaged over many repeats of the double-pulse excitation and many different relative protein-dye orientations) that are required for proceeding with Eqs. 5.4 and 5.5:

$$
\left\langle\kappa_{1} \kappa_{2}\right\rangle_{t}=\int d \vec{r} \int d \omega \int d \Omega \int d \Omega^{\prime}\left[R^{-1}(\Omega) U_{2}(\omega, \vec{r})\right] \cdot G\left(\Omega, \Omega^{\prime}, t\right)\left[R^{-1}\left(\Omega^{\prime}\right) U(\omega, \vec{r})\right]
$$


where $R^{-1}$ is the back-rotation operator. The integrations run over all possible initial and final orientations $\Omega^{\prime}$ and $\Omega$ of the protein, all possible dye-label orientations $\omega$, and all possible positions $\vec{r}$. It should be emphasized that the above expression is quite general, allowing for different excitation and detection geometries/polarizations for the first and second laser pulse.

In general a molecule can be modeled by an object with three orthogonal axes of rotation (principal axes) with three different rotational diffusion constants $\left(D_{\mathrm{a}}, D_{\mathrm{b}}\right.$, and $\left.D_{\mathrm{c}}\right)$ around each of these axes. For the sake of simplicity, I will further consider the special case of a symmetric top rotor where one has $D_{\mathrm{a}}=D_{\mathrm{b}}=D_{\perp}$ and $D_{\|}=D_{\mathrm{c}}$. Now, using the transformation relation (Eq.A.8), Green's function $G\left(\Omega, \Omega^{\prime}, t\right)$ (Eq.A.13) and the orthonormality of the eigenfunctions $C_{\mathrm{mk}}^{1}(\phi, \theta, \psi)$ (Eq.A.11) and of spherical harmonics $Y_{\mathrm{lm}}$ (Eq.A.20) (see appendix), the integrations over $\Omega, \Omega^{\prime}$ and $\omega$ can be performed analytically, resulting in

$$
\left\langle\kappa_{1} \kappa_{2}\right\rangle_{t}=\sum_{l=0}^{\infty} \sum_{m=-l}^{l}\left[\int d \vec{r} u_{2, l m}^{*}(\vec{r}) u_{1, l m}(\vec{r})\right] \cdot \exp \left[-l(l-1) D_{\perp} t-\left(D_{\mathrm{II}}-D_{\perp}\right) m^{2}\right]
$$

where $l, m$ are eigenvalues of the angular momentum operator defined by Eqs. A.5 and A.6 (see appendix). For a spherically symmetric molecule with $D_{\perp}=D_{\|} \equiv D$ this expression simplifies to

$$
\left\langle\kappa_{1} \kappa_{2}\right\rangle_{t}=\sum_{l=0}^{\infty} \sum_{m=-l}^{l}\left[\int d \vec{r} u_{2, l m}^{*}(\vec{r}) u_{1, l m}(\vec{r})\right] \cdot \exp [-l(l+1) D t]
$$

The explicit calculation of the coefficients $u_{\alpha, l m}(\vec{r})$ is a tremendous task, and for the details I refer the reader to (Enderlein et al., 2005) and citations therein. Remarkably, when neglecting optical saturation (i.e. excitation rate is directly proportional to the absolute square of the scalar product of the excitation light electric field amplitude times the molecule's absorption dipole vector), only coefficients with $l=(0,2,4)$ will differ from zero. Even taking into account depolarization in excitation and detection caused by objectives with high numerical aperture (Bahlmann et al., 2000a; Bahlmann et al., 2000b) does not change the computation noticeably. 


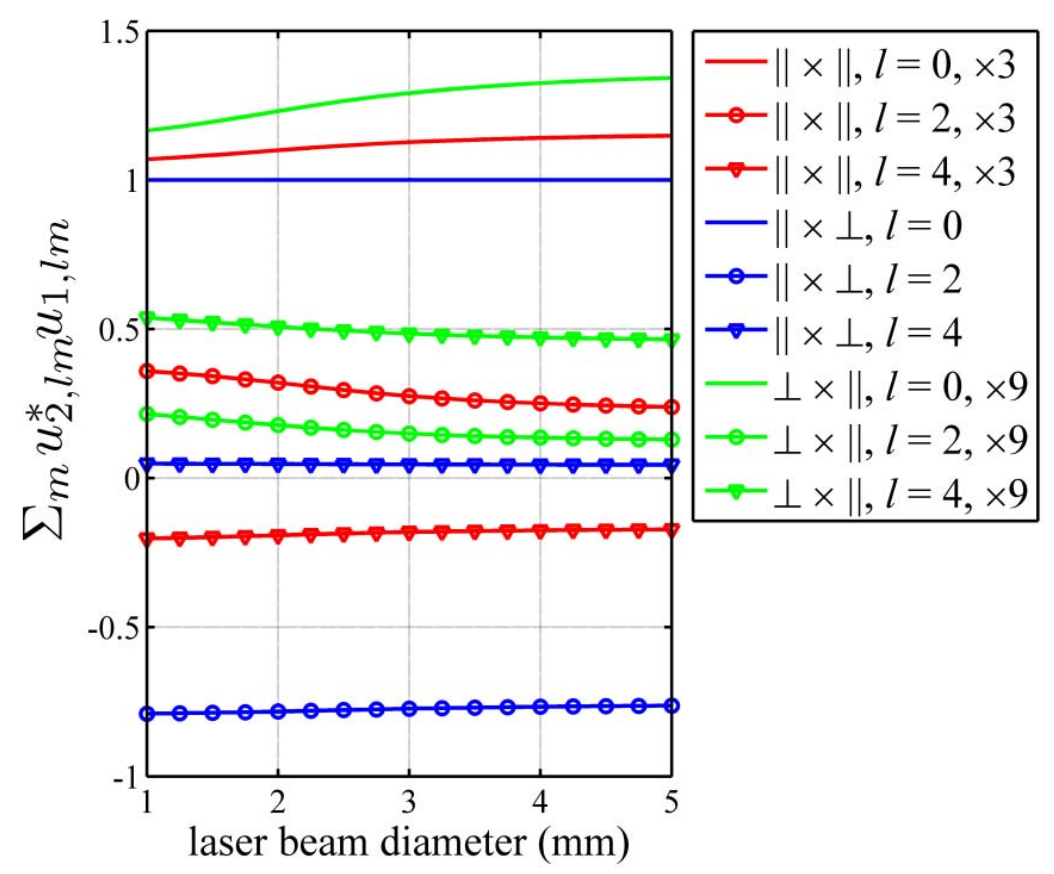

Fig.5.1: Dependence of the (normalized) coefficients $\left[\sum_{m} \int \mathrm{dr} u_{2, I m}^{*} \cdot u_{1, l m}\right]$ in Eq.5.8 for $l=0$ (solid lines), $I=2$ (solid lines with circles), $I=4$ (solid lines with triangles) and for different excitation/detection polarizations as a function of laser beam diameter (measured at the objective's back focal plane). It is assumed that detection is done through two polarizers with orthogonally aligned polarization axes for the first and the second photon. The red curves show the case when the first and second laser pulse are both polarized along the same direction as the first or the second detector polarizer; the blue curves show the case when both laser pulses have the same polarization as the corresponding detector polarizers; and the green curves show the case when both laser pulses are polarized perpendicular to the corresponding detector polarizers. The calculations were done for a perfectly aplanatic 1.2 N.A. water immersion objective.

As an example, Fig.5.1 shows the result of a numerical calculation for a 1.2 N.A. water immersion objective as a function of the laser beam diameter coupled into the objective's back focal plane. In these calculations, it was assumed that detection is done by two detectors looking at orthogonal emission polarizations. Without loss of generality, I will denote the detection polarization for the first photon by the symbol $\|$, and that for the second photon by $\perp(\| \times \perp$ detection polarization mode). Then, there are three principally different excitation modes: (i) polarization of excitation for the first and second photon is both parallel to the respective detection polarization $(\| \times \perp$ excitation polarization mode), (ii) polarization of excitation for the first and second photon is both orthogonal to the respective detection polarization $(\perp \times \|$ excitation polarization mode), and (iii) excitation polarization for both photons is the same $(\|\times\|$ or $\perp \times \perp$ excitation polarization mode), so 
that the first (second) photon is excited with an excitation polarization parallel to its detection polarization, and the second (first) orthogonally to its detection polarization. Fig.5.1 shows several remarkable features: Firstly, the amplitude ratios in the $\| \times \perp$, the $\perp \times \|$ and the $\|\times\|$ excitation mode are close to $9: 1: 3$ for $l=0,(-18): 1: 3$ for $l=2$, and $(-6): 8: 9$ for $l=4$, which are the values in the limit of zero numerical aperture, the situation considered by Aragón and Pecora (1975). Secondly, one has always non-zero contributions with $l=4$. However, the relative weight of these contributions when compared to the $l=2$ term is smallest for the $\| \times \perp$ excitation mode, where it is ca. $1 / 15^{\text {th }}$ of the amplitude for $l=2$.

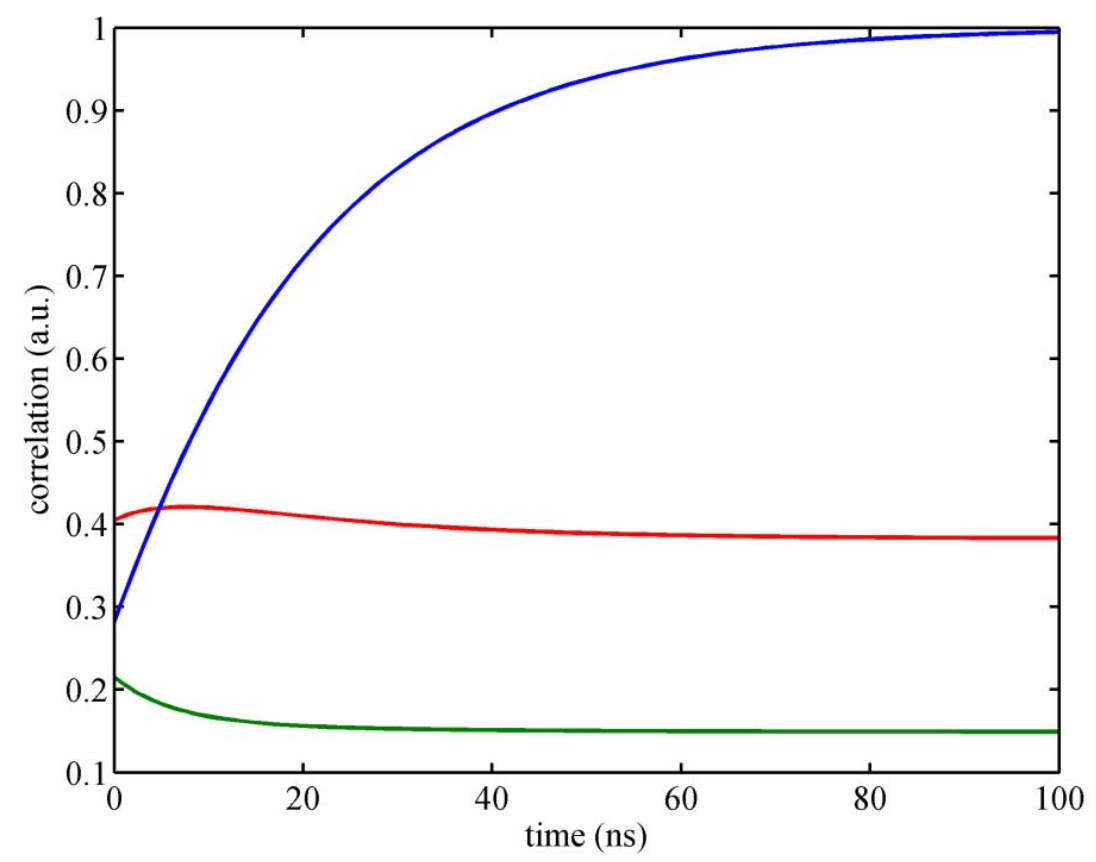

Fig.5.2: Correlation functions for $\| \times \perp$ (blue), $\perp \times \|$ (green) and $\|\times\|$ (red) excitation mode for a spherical globular protein with 20 ns rotational diffusion time.

Thirdly, when getting closer to diffraction-limited focusing (values at the right end of Fig.5.1), depolarization effects have a non-negligible impact on the different pre-exponential amplitudes in Eq.5.7. The lowest impact is observed for the $\| \times \perp$ excitation mode, which makes this mode of excitation/detection the most favorable one for measuring rotational diffusion via fluorescence correlation spectroscopy in a confocal microscope with high N.A. It yields maximum amplitude of the lag-time dependent part of the correlation function with 
smallest contribution from the $l=4$ mode and smallest impact from depolarization effects. As an example, the modeled correlation functions for a globular protein (isotropic rotor) with 20 ns rotational diffusion time $\tau_{\text {rot }}=1 / 6 D_{\text {rot }}$ are shown in Fig.5.2.

In many cases, fluorescent molecules exhibit a non-negligible angle between absorption and emission dipole. This will change the amplitudes of the different exponential terms in the autocorrelation function, but not the exponents themselves. Because the data analysis of autocorrelation curves for obtaining rotational diffusion values will solely rely on these exponents, I will not consider how a finite angle between absorption and emission dipole will modify the pre-exponential amplitudes.

\subsubsection{Molecular shape and rotational diffusion}

In this section I will briefly discuss when it is necessary to take into account the nonspherical shape of a molecule, and when the assumption of a rotationally symmetric shape is still sufficient. As already noted, any molecule can be modeled by an object with three orthogonal axes of rotation (principal axes) with, in the most general case, three different rotational diffusion constants around each of these axes. In almost all cases of practical interest, it is sufficient to approximate a molecule by a symmetric top, i.e. an object that has two identical rotational diffusion constants around two of its principal axes and one different around the third. This corresponds to approximating the shape of a molecule by a prolate or oblate spheroid of rotation. The question is how large the axis ratio between the axes of the spheroid has to be so that it is clearly discernible in a rotational diffusion measurement. Following Perrin (Perrin, 1934; Perrin, 1936) and Koenig (Koenig, 1975) the rotational diffusion coefficients for an oblate spheroid of rotation with aspect ratio $\varepsilon=R_{\perp} / R_{\|}<1$ are given by

$$
\frac{D_{\|}}{D_{0}}=\frac{3 \varepsilon^{2}}{2\left(1-\varepsilon^{4}\right)}\left\{\frac{2-\varepsilon^{2}}{\sqrt{1-\varepsilon^{2}}} \ln \left[\frac{1+\sqrt{1-\varepsilon^{2}}}{\varepsilon}\right]-1\right\}
$$

and

$$
\frac{D_{\perp}}{D_{0}}=\frac{3 \varepsilon}{2\left(1-\varepsilon^{4}\right)}\left\{1-\frac{\varepsilon^{2}}{\sqrt{1-\varepsilon^{2}}} \ln \left[\frac{1+\sqrt{1-\varepsilon^{2}}}{\varepsilon}\right]\right\}
$$


whereas for a prolate spheroid of rotation $(\varepsilon>1)$ they read

$$
\frac{D_{\|}}{D_{0}}=\frac{3 \varepsilon^{2}}{2\left(\varepsilon^{4}-1\right)}\left\{\frac{\varepsilon^{2}-2}{\sqrt{\varepsilon^{2}-1}} \arctan \left(\sqrt{\varepsilon^{2}-1}\right)+1\right\}
$$

and

$$
\frac{D_{\perp}}{D_{0}}=\frac{3}{2\left(\varepsilon^{2}-1\right)}\left\{\frac{\varepsilon^{2}}{\sqrt{\varepsilon^{2}-1}} \arctan \left(\sqrt{\varepsilon^{2}-1}\right)-1\right\}
$$

Here, $D_{0}$ is the diffusion coefficient of a sphere of radius $R_{0}$ with the same volume as the spheroid, i.e.

$$
R_{0}^{3}=R_{\|} R_{\perp}^{2}
$$

and the value of $D_{0}$ is given by Eq.5.1. In all the above expressions, the subscript $\|$ refers to the symmetry axis, and the subscript $\perp$ to the two transversal axes of the spheroid. Fig.5.3 shows the dependence of the two rotational diffusion coefficients on the eccentricity $\varepsilon$ of the spheroid.

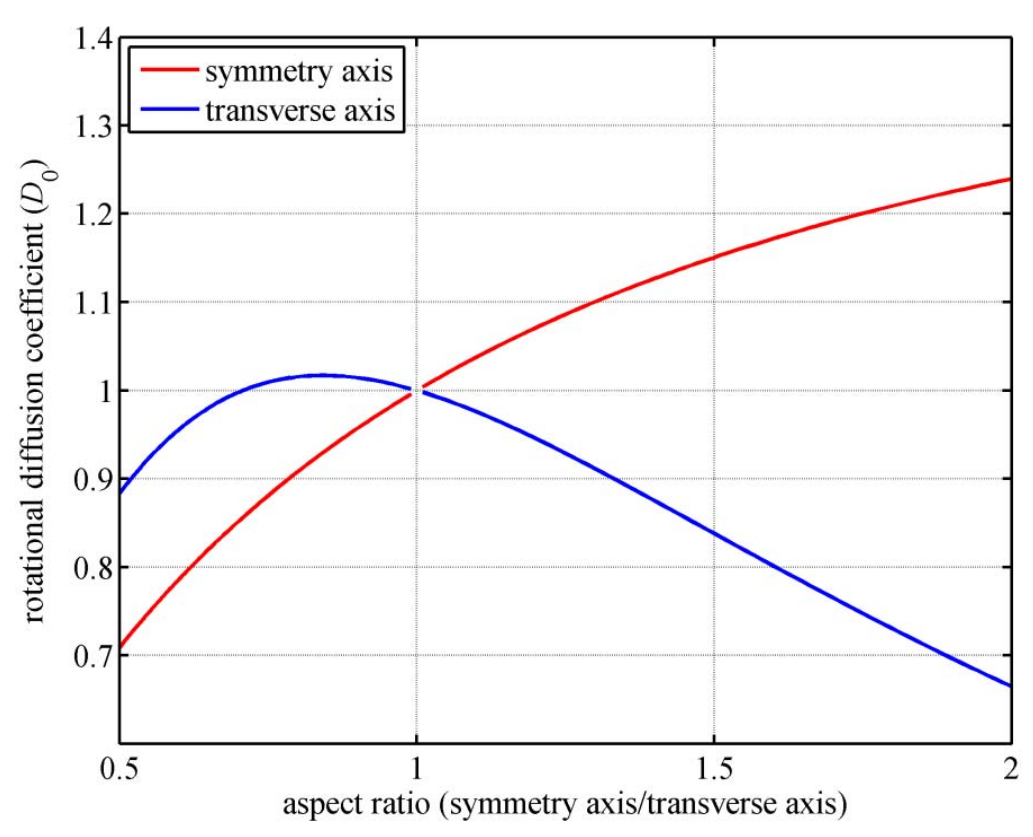

Fig.5.3: Dependence of the rotational diffusion coefficients on spheroid eccentricity. 
As can be seen, the values of rotational diffusion coefficients change quite quickly with changing eccentricity. Theoretically, it should be possible to observe the difference in the rotational diffusion coefficients around the symmetry and the transverse axes by the emergence of a more complex multi-exponential behavior of the correlation function compared to the correlation function produced by an ideal spherical rotor.

However, in practice the measured correlation curves are usually too noisy to extract that information if the axis ratio becomes not exceedingly large. Usually one fits the correlation function assuming a spherically-shaped molecule and obtains a mean rotational diffusion coefficient and a mean hydrodynamic radius. This corresponds to taking the mean of the diffusion coefficients, $\langle D\rangle=\left(2 \cdot D_{\perp}+D_{\|}\right) / 3$, and to use Eq.5.1 for obtaining the hydrodynamic radius. Due to the cubic relationship between radius and diffusion coefficient, the dependence of the thus-defined mean value of hydrodynamic radius changes much less with eccentricity than the individual rotational diffusion coefficients. This is shown in Fig. 5.4 , where one can see that the mean value of the hydrodynamic radius changes only slightly in the range of $0.75<\varepsilon<1.5$ at maximum by only $2 \%$. Thus, assuming a spherical shape is a quite reasonable approach for moderate values of eccentricity. I will use this assumption when measuring the rotational diffusion of globular proteins.

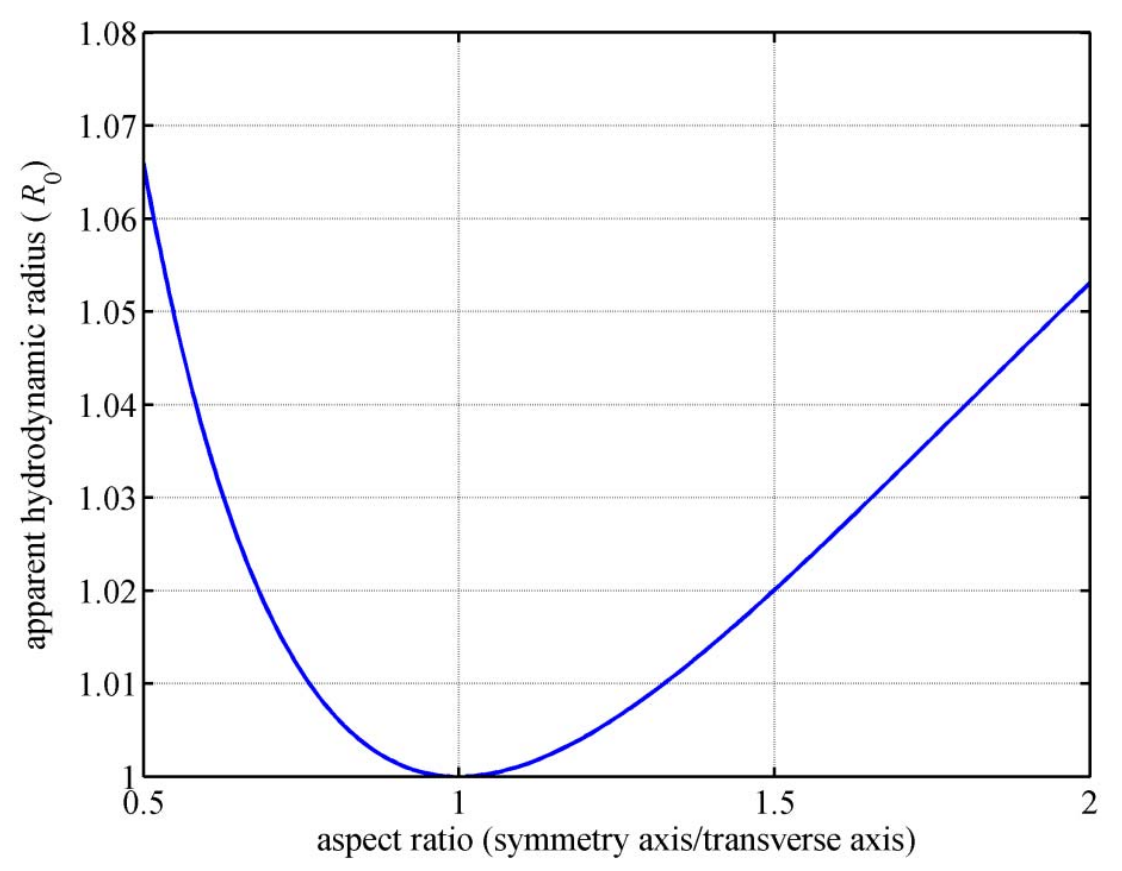

Fig.5.4: Dependence of the mean hydrodynamic radius on spheroid eccentricity. 


\subsection{Materials and methods}

\subsubsection{Measurement set-up}

All measurements were done with the same confocal microscopy system (MicroTime 200 with dual-focus option, PicoQuant GmbH, Berlin, Germany) described in chapter 2. To measure rotational diffusion a few minor changes in set-up were made: The system was used without the DIC prism. Thus all measurements were done with singlefocus FCS. Two red laser diodes are pulsed synchronously with the highest possible repetition rate of $80 \mathrm{MHz}$. To create a pulsed interleaved excitation (PIE) with about 6 nsspaced pulses of alternating polarization, a time delay of 6 ns (Ortec Delay 425, AMETEK $\mathrm{GmbH}$, Meerbusch, Germany) is inserted between the pulse trains of the first and second laser.

The short delay of $6 \mathrm{~ns}$ between the laser pulses requires a fluorescent label with sufficiently short lifetime assuring that the fluorescence excited by one laser pulse completely decays until the next excitation pulse. Therefore I chose Alexa Fluor ${ }^{\circledR} 647$ succinimidyl ester (Invitrogen $\mathrm{GmbH}$, Karlsruhe, Germany) and Cy5 bis-succinimidyl ester (GE Healthcare Europe GmbH, Freiburg, Germany), which have fluorescence lifetimes about $1 \mathrm{~ns}$.

Sample temperature was controlled with a HH500 digital thermometer (Omega Newport Electronics GmbH, Deckenpfronn, Germany). The values of the rotational diffusion coefficient and resulting hydrodynamic radius were subsequently recalculated for a temperature of $20^{\circ} \mathrm{C}$ employing Eq.5.1, and using the known dependency of water's dynamic viscosity on temperature.

\subsubsection{Calculation of the ACF}

As described in the theory section, the most advantageous mode of measuring an ACF for determining rotational diffusion is to calculate it from photon pairs excited with laser pulses of crossed polarization $(\| \times \perp)$ and detected with two detectors having detection polarization collinear to the corresponding excitation pulses (i.e. $\| \times \perp$ detection polarization mode). This is relatively easy to achieve with the experimental set-up and the described measurement mode. To better understand that, consider the TCSPC histograms as recorded by both detectors in the set-up, which are shown in Fig.5.5. As can be seen, each detector observes two consecutive fluorescence decays within a complete excitation cycle: one with 
a large and one with small amplitude. The large amplitude decay corresponds to a laser pulse polarization collinear with the detection polarization, whereas the small amplitude decay corresponds to a laser pulse polarization orthogonal to the detection polarization. Thus, by inspecting the TCSPC histograms, one can precisely determine the relative polarizations of the exciting laser pulse with respect to the detection polarization.

Using fluorescence dyes showing fluorescence decay times sufficiently short so that their fluorescence has nearly completely decayed until the next laser pulse occurs, and exploiting the TCSPC information of each photon, one can unequivocally associate each detected photon to the laser pulse which had excited it, similarly to what is done in pulsed interleaved excitation (Müller,B.K. et al., 2005) or dual-focus FCS (Dertinger et al., 2007).

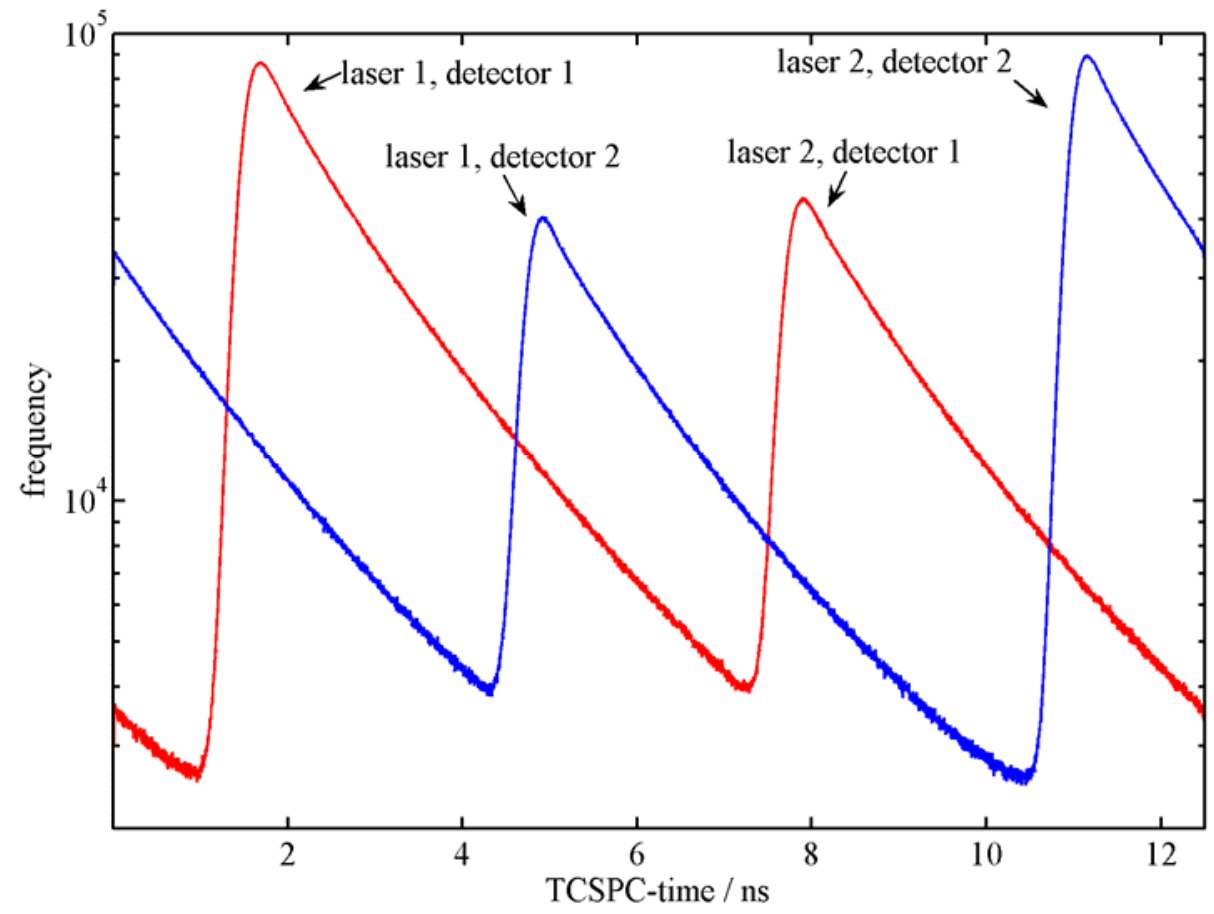

Fig.5.5: Red curve shows TCSPC-histogram of photons detected by detector \#1, in blue is the corresponding curve for detector \#2. The maxima at $1.75 \mathrm{~ns}$ (detector \#1) and $11.5 \mathrm{~ns}$ (detector \#2) correspond to laser pulses with their polarization collinear with the detection polarization. The local maxima at $8.0 \mathrm{~ns}$ (detector \#1) and $5.25 \mathrm{~ns}$ (detector \#2) correspond to laser pulses with their polarization orthogonal to the detection polarization.

Now, having the ability to determine, for each detected photon, the polarization of its exciting laser pulse, and the polarization of its detector, it is straightforward to calculate the desired $\| \times \perp$-polarization ACF by correlating all photon pairs where the first photon is 
excited by a || polarized laser and detected by the detector with || detection polarization, and the second photon is excited by a laser pulse with $\perp$ polarization and detected by the detector with $\perp$ detection polarization. This computation is done using a general algorithm of calculating an ACF on the basis of asynchronous photon counting data as described in Wahl et al. (2003).

At this point it is useful to realize that for extracting the rotational diffusion information from the ACF it is not necessary to compute the ACF with a temporal resolution better than that which is given by the laser pulse distances. Therefore, each detected photon is assigned with a virtual detection time equal to the time of its exciting laser pulse. By doing that, the resulting correlation function loses all information connected with the fluorescence decay, but maintains the rotational diffusion information. A resulting ACF is shown in Fig.5.6, where the bar plot shows values only at the discrete lag times corresponding to all possible time intervals between orthogonal and horizontal laser pulses. Here, ACF values for $t>t_{0}$ correspond to photon pairs where the first photon is detected by the detector \#1 and the second by detector $\# 2$, and ACF values for $t<t_{0}$ to the reverse order of detection, where $t_{0}$ is some absolute time offset determined by the relative temporal position of the laser pulse trains with respect to the internal clock of the photon counting electronics. Of course, for both $t>t_{0}$ and $t<t_{0}$, only photon pairs are correlated where the pulse polarization is collinear with detection polarization. The representation of the ACF as shown in Fig.5.6 considerably simplifies its evaluation, because the visible temporal dynamics is only due to rotational diffusion but not to fluorescence decay.

Fitting is done with a mono-exponential function of the form

$$
A+B \exp \left(-6 D\left|t-t_{0}\right|\right)
$$

where $A$ and $B$ are some amplitude factors, and $D$ is the rotational diffusion coefficient. By adopting this fit function it is assumed that the studied molecules are close to spherically symmetric, and that all terms with $l>2$ in Eq.5.8 are negligible compared with the $l=2$ term.

\subsection{Results and discussion}

First, I measured the rotational diffusion of HSA non-specifically labeled with Alexa Fluor $^{\circledR} 647$ as described in section 4.4. Fig.5.6 shows the ACF for the $\| \times \perp$ excitation mode, 
calculated as described in the previous section. The data are fitted with the mono-exponential model curve of Eq.5.14, the exponent of which yields the inverse rotational diffusion time, $\tau_{\text {rot }}=1 / 6 D_{\text {rot. }}$ As can be seen, the fit quality is remarkably good, although a spherical symmetry of the protein is assumed and any terms with $l=4$ are neglected. This exemplifies once more that the $l=4$ contribution to the correlation function in $\| \times \perp$ excitation mode is indeed negligibly small.

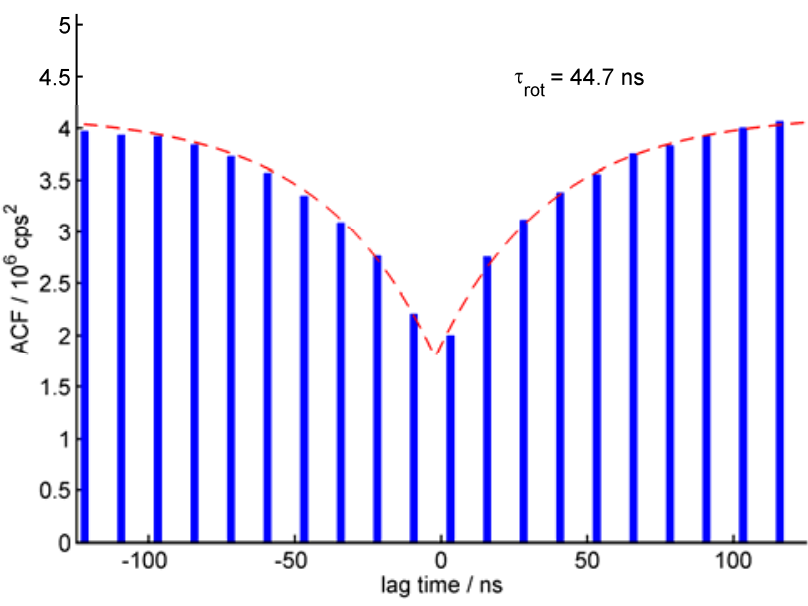

Fig.5.6: Measured $\| \times \perp$-correlation function (blue bars) and fitted mono-exponential lag-time dependence (dashed red line) for HSA.

To check the dependence of the

obtained rotational diffusion time $\tau_{\text {rot }}$ on total measurement time, I partitioned the measured photon stream into subsets of different measurement times, calculated for each subset the correlation function, fitted the rotational diffusion time, and averaged these values over subsets of equal duration. The resulting values of $\tau_{\text {rot }}$ and their standard deviation (if the total amount of data could be divided into more than two subsets for the given duration) are shown in Fig.5.7. As can be seen, the obtained value of rotational diffusion quickly approaches a fixed value if the measurement time becomes lager than 2000 s. Because the average photon count rate of the measurement was $\sim 43$ kcps (both detectors), this corresponds to a value $\sim 10^{8}$ measured photons.

Using the Stokes-EinsteinDebye equation, Eq.5.1, and the known values of temperature and viscosity, the determined rotational diffusion value corresponds to a value of the hydrodynamic radius $R_{\text {rot }}$ of

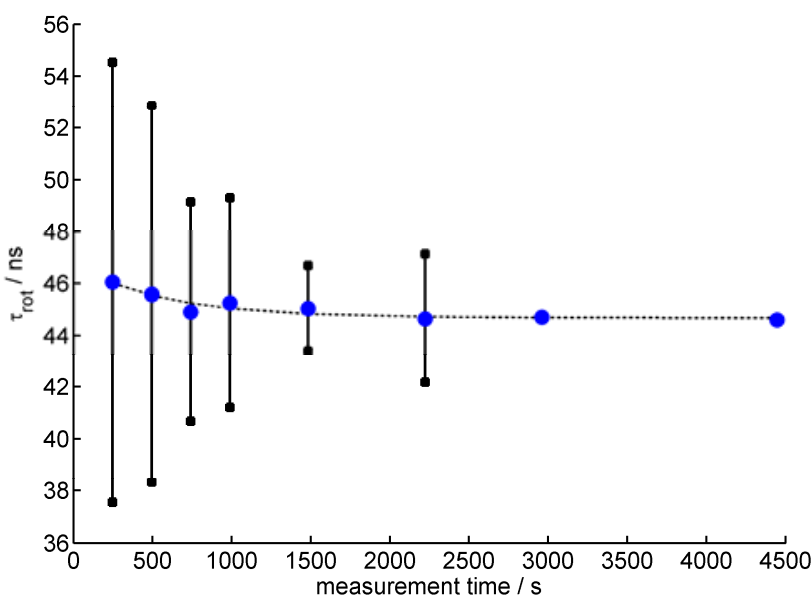

Fig.5.7: Dependence of the determined values of rotational diffusion on measurement time. The dotted line shows an exponentially decaying asymptotic fit to the determined values 
$(36.1 \pm 0.4) \AA$.

Next, I measured the rotational diffusion of the protein bovine serum albumin (BSA), again non-specifically labeled with Alexa Fluor ${ }^{\circledR}$ 647. The resulting ACF is shown in Fig.5.8, together with a mono-exponential fit.

The dependence of the obtained value on measurement time was similar to that for BSA, and the finally obtained hydrodynamic radius is $(36.9 \pm 0.5) \AA$, which is in good agreement with literature values for

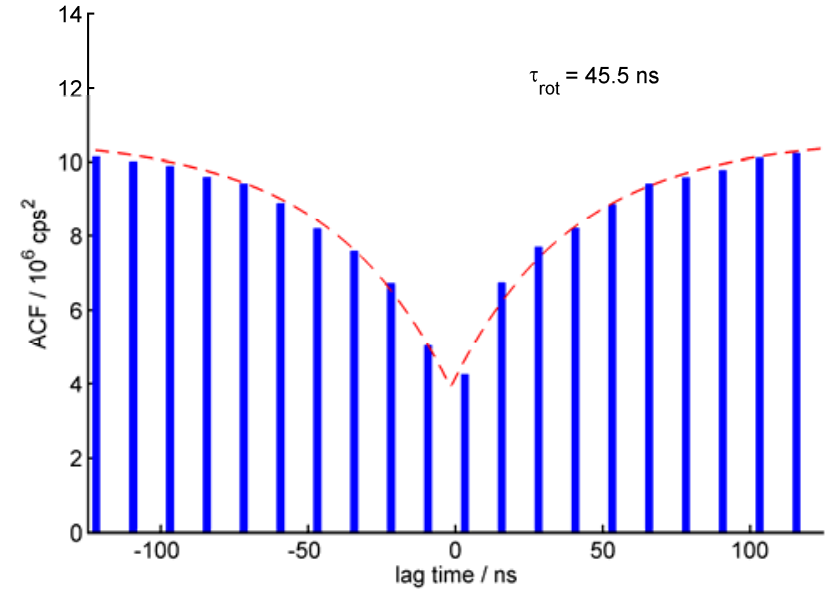

Fig.5.8: Measured $\| \times \perp$-correlation function (blue bars) and fitted mono-exponential lag-time dependence (dashed red line) for BSA. BSA $\left(\mathrm{R}_{\mathrm{rot}}=34 \AA\right.$ in Flecha \& Levi (2003) and $\mathrm{R}_{\text {rot }}=35 \AA$ according to Murtaza et al. (1999)), where it was measured via fluorescence anisotropy. In an extended study, Ferrer et al. (2001) recently combined both fluorescence anisotropy measurements with theoretical modeling to elucidate the anisotropic

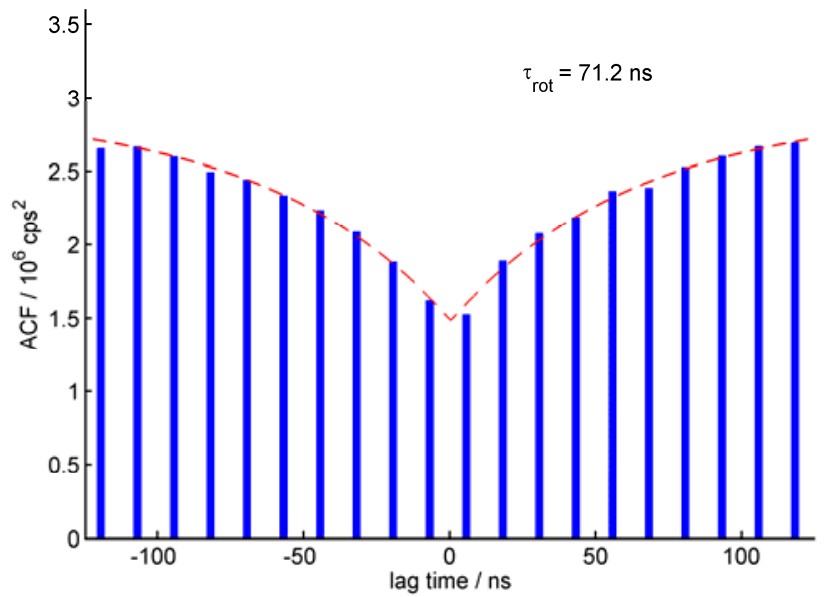

Fig.5.9: Measured $\| \times \perp$-correlation function (blue bars) and fitted mono-exponential lag-time dependence (dashed red line) for aldolase shape of BSA in solution. They found an average radius value of $37.5 \AA$, which is still in reasonable agreement with the value found by the new method.

Finally, I measured the rotational diffusion of the nonspecifically labeled proteins aldolase and ovalbumin. Here, the label Alexa Fluor $^{\circledR} 647$ is not sticky enough to corotate with the proteins. It is assumed that the hydrophobicity of BSA accidentally assured such a corotation, but that it is not granted when labeling arbitrary proteins. Thus, I chose the bisfunctional fluorescence label Cy5 bis-succinimidyl ester for non-specifically labeling aldolase and ovalbumin. By fluorescence anisotropy measurements I verified that this label 
indeed co-rotates with the proteins. The measured ACFs are presented in Fig.5.9 and 5.10. For these two proteins, fit quality was also excellent, and the extracted hydrodynamic radius values are $(41.2 \pm 0.3) \AA$ for aldolase and $(28.6 \pm 0.7) \AA$ for ovalbumin. For both proteins, I observed a similar dependence of fitted values on measurement time as those observed for BSA.

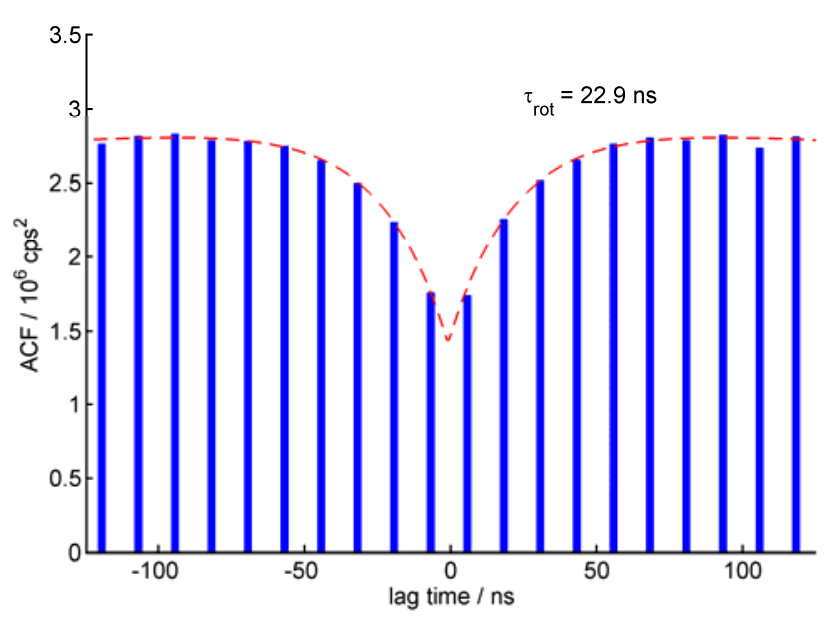

Fig.5.10: $\quad$ Measured $\| \times \perp$-correlation function (blue bars) and fitted mono-exponential lag-time dependence (dashed red line) for ovalbumin. To take into account the early onset of triplet-state pumping, a second exponent was used for fitting.
The following table summarizes all the results on the hydrodynamic radii of the studied proteins, and compares it with literature values (as far as they were available) and with theoretical predictions by HydroPro 7c (de la Torre et al., 2000) as in section 4.3. The information about protein structure was taken from the protein database (entry indicated in brackets). There is no theoretical value for BSA, because no crystal structure is known for that protein.

Table 5.1: Values of proteins hydrodynamic radius.

\begin{tabular}{ccccc} 
Protein & \multicolumn{3}{c}{$R_{\text {rot }} / \AA$} & MW / kDa \\
\hline FCS & Literature & HydroPro 7c & \\
& $36.1 \pm 0.4$ & & $36(1 \mathrm{bm} 0)$ & 69.4 \\
BSA & $36.9 \pm 0.5$ & 34 & not avail. & 66.0 \\
& & 37.5 & & \\
Ovalbumin & $28.6 \pm 0.7$ & 32 & $31(1 \mathrm{vac})$ & 44.2 \\
Aldolase & $41.2 \pm 0.3$ & & $49(1 \mathrm{zah})$ & 156.8
\end{tabular}


In summary, I presented a new variant of fluorescence correlation spectroscopy to measure the rotational diffusion of macromolecules. The approach ensures (i) a maximum amplitude of the rotational-diffusion related contribution in the correlation function, (ii) a minimal impact of higher order $(l=4)$ contributions, allowing for a mono-exponential fitting of the rotational diffusion time, and (iii) it works best for rotational diffusion times that are large compared to the fluorescence decay time, exactly the situation when fluorescence anisotropy will no longer be useful. Thus, it can be expected that fluorescence correlation spectroscopy on the nanosecond timescale will become be an efficient and reliable method for measuring rotational diffusion of large macromolecules. 



\section{Summary}

The main goal of my thesis was to size molecules with high precision using two methods based on fluorescence correlation spectroscopy (FCS): dual-focus FCS and nanosecond time-scale FCS. Size is one of the basic characteristics of molecules. The high accuracy in size determination is required because many biologically relevant changes of the molecular size (such as the conformational changes of proteins) happen in the order of Ångstrøm. The ability to determine the molecular size with such an accuracy can be applied to study molecular interactions or intramolecular reorganization upon temperature changes, for example.

In a dual-focus FCS (2fFCS), one of the core elements in the measurement set-up is the DIC prism that generates two laterally shifted foci. The distance between them (determined by the shear distance of the DIC prism) has to be determined as precisely as possible in order to achieve the required accuracy in $2 \mathrm{fFCS}$ measurements. The relative error of the shear distance leads to doubled relative error in the diffusion coefficient. Therefore I started my thesis by presenting a precise method for measuring the shear distance of DIC prism. The method is based on comparison of diffusion coefficient measured by $2 \mathrm{fFCS}$ and by dynamic light scattering. The achieved precision was less than $\pm 1.5 \mathrm{~nm}$ for shear distance values around $400 \mathrm{~nm}$, i.e. the overall accuracy of $2 \mathrm{fFCS}$ was about $1 \%$. Besides, this method can be useful for calibrating DIC microscopes as well as $2 \mathrm{fFCS}$ measurement systems.

The most important and disturbing source of inaccuracy and irreproducibility in conventional FCS measurements is the optical saturation phenomenon. Therefore I analyzed its influence on $2 \mathrm{fFCS}$ performance. It was shown by theoretical analysis that 2 fFCS was amazingly robust against ground-state-to-excited-state optical saturation, provided that the excitation conditions are optimal (not too close to the diffraction limit). In presence of a triplet-state saturation or photophysically similar processes, the theory showed that $2 \mathrm{fFCS}$ should yield an increasing systematic error in the diffusion coefficient with increasing excitation intensity. However, it was found out that $2 \mathrm{fFCS}$ experimental results were surprisingly insensitive against optical saturation for low excitation powers (up to $40 \mu \mathrm{W}$ per laser focus) and relaxed laser focusing (not too close to the diffraction limit). The reasons behind this are not completely understood and require further investigations. Until then, the dependence of the diffusion coefficient on excitation intensity in 2fFCS should be determined experimentally even under optimal excitation conditions. 


\section{Molecular sizing with dual-focus FCS}

2fFCS was applied for determining accurate hydrodynamic radii of molecules at pico- to nanomolar concentrations. The high accuracy of the method was exemplified on measuring diffusion coefficients values of Atto655 free acid and its two derivatives NHS-ester and maleimide. It was shown that $2 \mathrm{fFCS}$ was able to resolve side group variations leading to diffusion coefficient differences of about $4 \%$. This difference corresponded to the molecular weight variation of $15-20 \%$ and, therefore, can be considered as the lower boundary of $2 \mathrm{fFCS}$ sensitivity.

Next, it was observed that the diffusion behavior of small molecules was not only determined by their molecular weight but also by their shape and flexibility. This is nicely illustrated by the diffusion behavior of fluorescent dyes. It was found that the diffusion behavior of different derivatives of Atto655 could be described by the MarkHouwink-Kuhn-Sakurada (MHKS) power law with an exponent of 0.333 . This value of the exponent is characteristic for a globular molecule with spherical shape. However Cy5 and Alexa Fluor ${ }^{\circledR} 647$ exhibited the diffusion behavior shifted to that of random coil conformation, characterized by an exponent of 0.5 in the MHKS equation. These two dyes have a much more flexible structure than Atto655. Therefore, they exhibit a slower diffusion coefficient than that of a sphere of the same molecular weight, and their diffusion coefficient lies between the limiting values for a sphere and a random coil.

Subsequently, I measured the diffusion coefficients of short oligopeptides with amino-acid sequences going from one to eight amino-acids of the FKPYDAAD sequence. It was shown that the accuracy of $2 \mathrm{fFCS}$ allowed to distinguish with certainty between oligopeptides that differed by as few as two amino acids. Considering that the oligopeptide FKPYDAA is a short peptide with a cleavage site for pepsin (digestive protease), 2fFCS can be applied to identify cleaved fragments of oligopeptide after the pepsin treatment. This can be of use for developing assay for diagnostics in medicine.

Besides, the quantitative relation between molecular weight of those oligopeptides and their measured diffusion coefficients was analyzed. Interestingly, it was discovered that the complex molecule (consisting of oligopeptides, Alexa Fluor ${ }^{\circledR} 647$, and the short linker) could be described by a very simple model of an ideal freely joint chain. This makes it a rare occurrence when an idealized model describes the behavior of a real molecule with very good accuracy.

Next, 2fFCS was used to size common globular proteins. The accuracy of hydrodynamic radii determination varied from $2 \%$ to $5 \%$. The results were found to be 
in good agreement both with literature values and with theoretical calculations based on known crystal structure of the proteins.

Two proteins such as bovine serum albumin (BSA) and $\alpha$-aldolase were labeled with either Atto647N or Alexa Fluor ${ }^{\circledR} 647$. The hydrodynamic radius of $\alpha$-aldolase was found to be independent on which dye was used for labeling. However BSA hydrodynamic radius in BSA-Atto647N complex was $8 \%$ bigger than that in BSAAlexa Fluor ${ }^{\circledR}$ 647. Hence it was identified that dye interaction with BSA can introduce conformational changes to it. Therefore, it is essential to check how a protein behaves with respect to labeling with different dyes so that the dye-specific effects can be accounted for.

\section{Molecular sizing with FCS on nanosecond time scale}

Another fundamental dynamic process of molecules within a solution is thermally induced rotational diffusion, which happens on a nanosecond time scale for macromolecules. A new variant of fluorescence correlation spectroscopy to measure the rotational diffusion coefficient was introduced as a final chapter of my thesis. By using pulse interleaved excitation with alternating polarization, it becomes possible to pick out the correlation function with maximal rotational diffusion contribution. Further, higherorder orbital momentum quantum number $(l=4)$ contribution can be neglected, allowing for mono-exponential fitting of the rotational diffusion kinetics, greatly simplifying the data analysis. Finally, this method works best for rotational diffusion times much larger than the fluorescence decay time, in which case fluorescence anisotropy is no longer applicable.

Hence, the fluorescence correlation spectroscopy on the nanosecond time scale proves an efficient and reliable method for measuring rotational diffusion of large macromolecules and thus their hydrodynamic radius. Here, I applied this method to measure rotational diffusion of common globular proteins. The achieved precision was better than 0.5 Ångstrøm when the measurement time was long enough to record more than $2 \cdot 10^{8}$ photons per point. This yields the relative accuracy of about $1 \%$, which is comparable to pulsed field gradient NMR. 



\section{Appendix}

The general theory of rotational diffusion of an anisotropic rotor can be found in several textbooks on quantum mechanics and was, in the context of correlation spectroscopy and light scattering, developed by Aragón and Pecora in 1975, see also (Berne \& Pecora; 2000). In this section, only a brief review of the rotational diffusion theory will be presented. In particular, I focus on the case of symmetric top rotor. As in almost all cases of practical interest, it is sufficient to approximate the shape of a molecule by a prolate or oblate spheroid. In section A2, molecular detection function is described for further fluorescence correlation analysis of molecular rotation motion.

\section{A.1 Rotational diffusion equation}

The rotational diffusion equation

$$
\frac{\partial P}{\partial t}=-\left(D_{a} \hat{J}_{a}^{2}+D_{b} \hat{J}_{b}^{2}+D_{c} \hat{J}_{c}^{2}\right) P
$$

where $a, b$, and $c$ denote the principal axes of rotation of the molecule, $P=P(\psi, \theta, \phi)$ is the probability to find the molecule's principal axes rotated by Euler angles $\psi, \theta$ and $\phi$ with respect to the lab frame, the $D_{\mathrm{a}, \mathrm{b}, \mathrm{c}}$ are the generally different rotational diffusion coefficients around the molecule's principal axes, and the $\hat{J}_{\mathrm{a}, \mathrm{b}, \mathrm{c}}$ are the three angular momentum operators around these axes. Eq.A.1 is derived analogously to the more familiar translational diffusion equation. The difficulty with Eq.A.1 is that the angular momentum operators relate to the intrinsic frame of the molecule's principal axes which is rotating in time with respect to the fixed lab frame. To simplify matters, one can first rotate the molecule back to the lab's frame so that its axes align with the fixed Cartesian coordinate axes of the lab frame, then apply the operator, and finally rotate the molecule back, i.e.

$$
\frac{\partial P}{\partial t}=-R\left(D_{a} \hat{J}_{x}^{2}+D_{b} \hat{J}_{y}^{2}+D_{c} \hat{J}_{z}^{2}\right) R^{-1} P
$$

where $R$ denotes the operation of rotating the molecule's frame from an orientation aligned with the lab's Cartesian $x, y, z$-coordinates to its actual orientation as specified by the Euler angles $\psi, \theta$ and $\phi$, see Fig.A.1. 


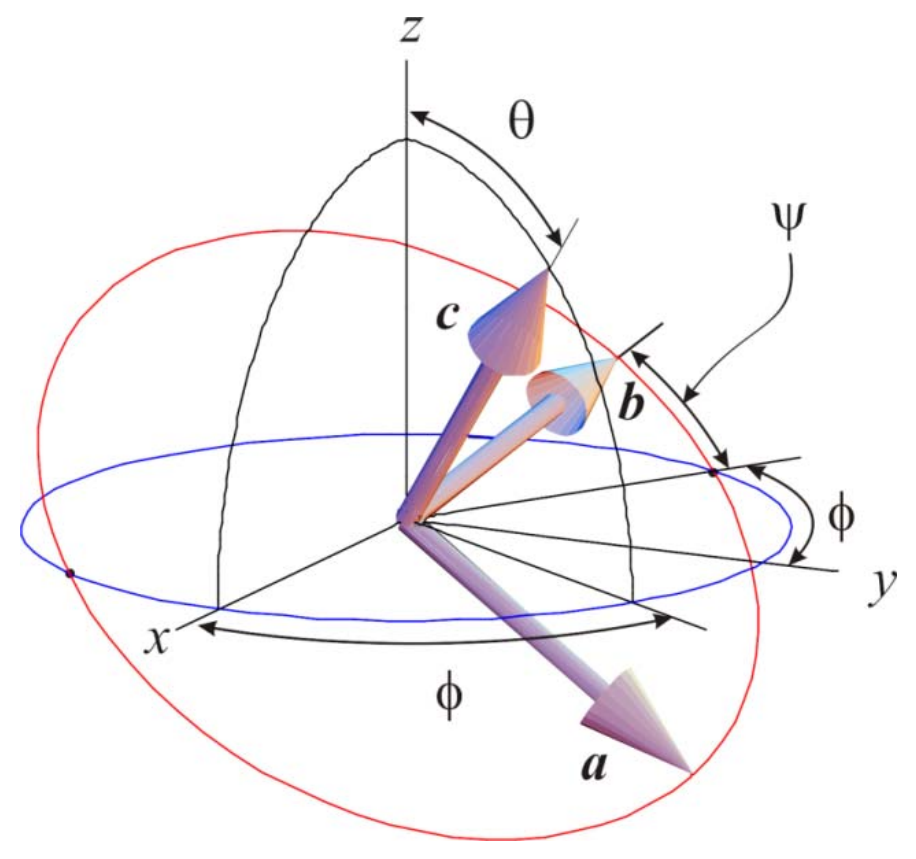

Fig.A.1: Geometric meaning of the three Euler angles $\phi, \theta$, and $\psi$. Shown are the molecule's three principal (and orthogonal) axes of rotation $a, b$, and $c$, and the three Cartesian axes $x, y$, and $z$ of the lab frame.

The rotation operator $R$ can be decomposed into

$$
R=R_{x}(\phi) R_{y}(\theta) R_{z}(\psi)
$$

where $R_{\mathrm{y}, \mathrm{z}}(\beta)$ denotes a rotation by angle $\beta$ around axis $y$ or $z$, respectively. The advantage of Eq.A. 2 is that the angular momentum operators are now referring to the fixed lab frame.

To further analyze Eq.A.2, let us consider the special case that the function $P$ is replaced by

$$
P=R|l, m\rangle
$$

where $|l, m\rangle$ is an eigenfunction of the angular momentum operator obeying the two relations

$$
\hat{\vec{J}}^{2}|l, m\rangle=\left(\hat{J}_{x}^{2}+\hat{J}_{y}^{2}+\hat{J}_{z}^{2}\right)|l, m\rangle=l(l+1)|l, m\rangle
$$

and 


$$
\hat{J}_{z}|l, m\rangle=m|l, m\rangle
$$

Inserting Eq.A.4 into Eq.A.2 yields

$$
\frac{\partial(R|l, m\rangle)}{\partial t}=-R\left(D_{a} \hat{J}_{x}^{2}+D_{b} \hat{J}_{y}^{2}+D_{c} \hat{J}_{z}^{2}\right)|l, m\rangle
$$

Next, one has to clarify how the rotation operator $R$ acts on $|l, m\rangle$. One of the most lucid derivations of this action is given by Feynman in (Feynman, 1964) using the possibility to represent any state $|l, m\rangle$ through a combination of spin-1/2 states for which the transformation relations under action of $R$ are well known, see e.g. chapter 3.3 in (Thompson, (1994)). The final result is given by

$$
R(\phi, \theta, \psi)|l, m\rangle=e^{i k \phi} S_{m k}^{l}(\theta)|l, k\rangle
$$

The functions $S_{\mathrm{mk}}^{\mathrm{1}}$ are Wigner's rotation matrices defined by

$$
\begin{aligned}
& S_{m k}^{l}(\theta)=\left\langle l, k\left|R_{y}(\theta)\right| l, m\right\rangle=\left[\frac{(j+k) !(j-k) !}{(j+m) !(j-m) !}\right]^{1 / 2} \ldots \\
& \cdot \sum_{n} \frac{(-1)^{j+k-n}(j+m) !(j-m) !}{k !(j+m-n) !(j+k-n) !(n-m-k) !} C^{2 n-m-k} S^{2 j+m+k-2 n}
\end{aligned}
$$

Here, the abbreviations $C=\cos (\theta / 2)$ and $S=\sin (\theta / 2)$ were used.

As initially mentioned I will restrict my considerations to the special case of a symmetric top rotor where one has $D_{\mathrm{a}}=D_{\mathrm{b}}=D_{\perp}$ and $D_{\|}=D_{\mathrm{c}}$. The general case of the fully asymmetric rotor will be shortly discussed later. For the symmetric top rotor, one finds, by multiplying Eq.A.7 with $\langle l, k|$, that the functions

$$
\exp \left\{-\left\lfloor D_{\perp} l(l+1)+\left(D_{\|}-D_{\perp}\right) m^{2}\right\rfloor \cdot t\right\} C_{m k}^{l}(\phi, \theta, \psi)
$$

with 


$$
C_{m k}^{l}(\phi, \theta, \psi)=c_{m k}^{l} \exp (i k \phi+i m \psi) S_{m k}^{l}(\theta)
$$

are eigenfunctions of the rotational diffusion equation. In Eq.A.8 a normalizing factor $c^{1}$ mk was introduced so that the $C_{\mathrm{mk}}^{\mathrm{l}}(\phi, \theta, \psi)$ represent a complete orthonormal system of eigenfunctions obeying the relations

$$
\int_{0}^{\pi} d \theta \sin \theta \int_{0}^{2 \pi} d \phi \int_{0}^{2 \pi} d \psi C_{m k}^{l}(\phi, \theta, \psi) C_{m k^{\prime} k^{\prime}}^{l^{\prime}}(\phi, \theta, \psi)=\delta_{l, l^{\prime}} \delta_{k, k^{\prime}} \delta_{m, m^{\prime}}
$$

Here, the $\delta_{1,1}$ are Kronecker symbols taking the value one for $l=1^{\prime}$ and zero otherwise. The orthogonality of the functions $C_{\mathrm{mk}}^{\mathrm{l}}(\phi, \theta, \psi)$ with respect to the variables $\phi$ and $\psi$ is obvious from their definition in Eq.A.8. The orthogonality with respect to $\theta$ is less obvious, but is a consequence of the fundamental orthogonality theorem of group theory (see e.g. Thompson, 1994) which is applied here to the functional representation of the three-dimensional rotation group as given by the functions $C_{\mathrm{mk}}^{1}(\phi, \theta, \psi)$. With this complete orthonormal system of eigenfunctions, the probability that a molecule has rotated, within time $t$, from an initial orientation $\Omega^{\prime}$ described by the Euler angles $\phi^{\prime}, \theta^{\prime}$ and $\psi^{\prime}$ into a final orientation $\Omega$ described by Euler angles $\phi, \theta$ and $\psi$ is given by Green's function in the standard way (Morse \& Feshbach, 1953) as

$$
G\left(\Omega, \Omega^{\prime}, t\right)=\sum_{l=0}^{\infty} \sum_{m, k=-l}^{l} \exp \left\{-\left[D_{\perp} l(l+1)+\left(D_{\|}-D_{\perp}\right) m^{2}\right] \cdot t\right\} C_{m k}^{l}(\phi, \theta, \psi) C_{m k}^{l^{*}}\left(\phi^{\prime}, \theta^{\prime}, \psi^{\prime}\right)
$$

where a star superscript denotes complex conjugation.

For the sake of completeness, I will briefly discuss the most general case of a completely asymmetric rotor. In this case it is not possible to obtain simple eigenfunctions of the form of Eq.A.1. However, it is helpful to introduce the operators

$$
\hat{J}_{ \pm}=\hat{J}_{x} \pm i \hat{J}_{y}
$$

so that the $\hat{\mathrm{J}}_{\mathrm{x}}$ and $\hat{\mathrm{J}}_{\mathrm{y}}$ operators on the right hand side of Eq.A.2 can be written as 


$$
\hat{J}_{x}^{2}=\frac{1}{4}\left(\hat{J}_{+}^{2}+\hat{J}_{-}^{2}+\hat{\vec{J}}^{2}-\hat{J}_{z}^{2}+\hat{J}_{z}\right)
$$

and

$$
\hat{J}_{y}^{2}=\frac{1}{4}\left(-\hat{J}_{+}^{2}-\hat{J}_{-}^{2}+\hat{\vec{J}}^{2}-\hat{J}_{z}^{2}+\hat{J}_{z}\right)
$$

where the commutation property of the angular momentum operators

$$
\left\lfloor\hat{J}_{x}, \hat{J}_{y}\right\rfloor \equiv \hat{J}_{x} \hat{J}_{y}-\hat{J}_{y} \hat{J}_{x}=i \hat{J}_{z}
$$

has been used. When taking into account how the operators $\hat{J}_{ \pm}$act on the eigenstates $|l, m\rangle$ (see e.g. chapter 3.4. in Feynman, 1964).

$$
\left.\hat{J}_{ \pm}|l, m\rangle=\sqrt{l(l+1)-m(m \pm 1)} l, m \pm 1\right\rangle
$$

it is straightforward to see that Eq.A.2 yields $2 l+1$ orthonormal eigenfunctions as superpositions of the states $|l, m\rangle$ with corresponding eigenvalues as characteristic temporal exponents, from which Green's function can be constructed as before. Because the case of a fully asymmetric rotor is of rather little interest for almost all fluorescence-based measurements of molecular rotation, I will not pursue this topic further. 


\section{A.2 Molecular detection function}

The so-called molecule detection function (MDF) describes the chance to excite and detect a photon for a dye molecule with a given orientation and position in sample space. The calculation of this function can be done using a wave-optics approach as described in ref. (Enderlein et al., 2005). For the following considerations it is important that the MDF can be expanded into a series of spherical harmonics in the angles $\alpha$ and $\beta$ which describe the angular orientation $\omega$ of the excitation/emission dipole (which are assumed to be collinear) as depicted in Fig.A.2.

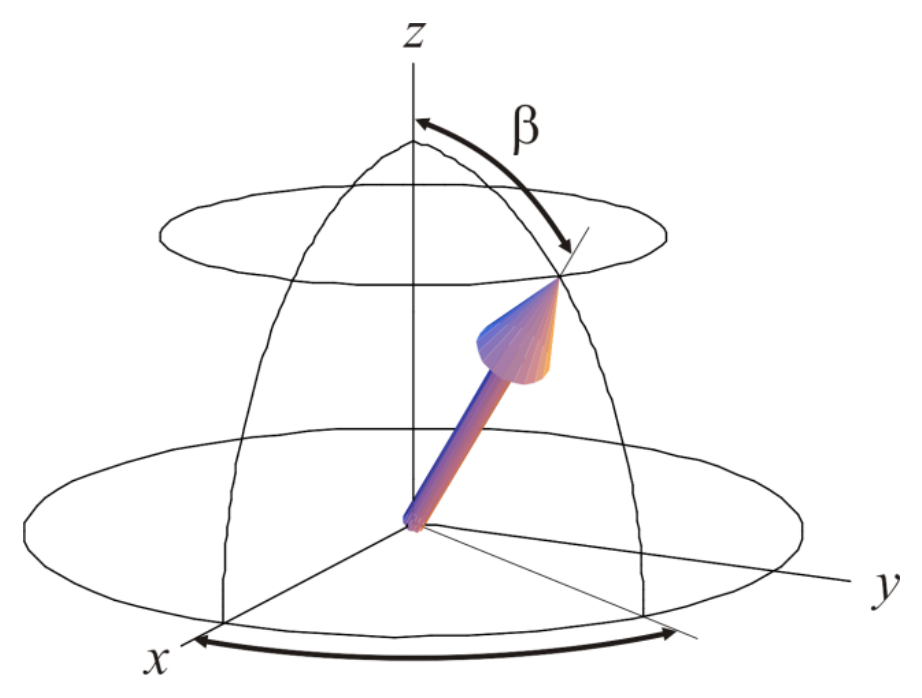

$\alpha$

Fig.A.2: Geometric meaning of the orientation angles $\alpha$ and $\beta$ with respect to the lab frame

The coefficients of this series expansion are functions of the molecule's position $\vec{r}$, and the MDF, which will be denoted by $U(\alpha, \beta, \vec{r})$ is thus represented through

$$
U(\omega, \vec{r}) \equiv U(\alpha, \beta, \vec{r})=\sum_{l=0}^{\infty} \sum_{m=-l}^{l} u_{l m}(\vec{r}) Y_{l m}(\beta, \alpha)
$$

where the spherical harmonics $Y_{\operatorname{lm}}(\beta, \alpha)$ are defined by

$$
Y_{l m}(\beta, \alpha)=P_{l}^{|m|}(\cos \beta) \exp (i m \alpha)
$$


Here, the functions $P_{l}^{|m|}(\cos \beta)$ are associated Legendre polynomials (Abramowitz, 1965). Using the orthogonality of spherical harmonics, the coefficients $u_{l m}(\vec{r})$ can be found from the full MDF via the backward transformation

$$
u_{l m}(\vec{r})=\int_{0}^{\pi} d \beta \sin \beta \int_{0}^{2 \pi} d \alpha U(\beta, \alpha, \vec{r}) Y_{l m}^{*}(\beta, \alpha)
$$

The importance of representation Eq.A.19 lies in the fact that the spherical harmonics themselves are representations of the three-dimensional rotation group and transform under rotation according to Eq.A.8.

The transformation relation (Eq.A.8), Green's function $G\left(\Omega, \Omega^{\prime}, t\right)$ (Eq.A.13) and the orthonormality of the eigenfunctions $C_{\mathrm{mk}}^{\mathrm{l}}(\phi, \theta, \psi)$ (Eq.A.11) and MDF (Eq.A.19) are used to get analytical solution of Eq.5.6 for the average of the product $\kappa_{1} \kappa_{2}$. This product is the most important part of correlation function giving by Eqs.5.4 and 5.5 (see section 5.1.1) as it contains the contribution of molecular rotational motion. 


\section{A.3 Summed up results for 2 fFCS and nanosecond time-scale FCS}

Table A.1: Diffusion coefficients and hydrodynamic radii for fluorescent dyes

\begin{tabular}{lccc} 
& $\mathrm{MW}^{+} / \mathrm{Da}$ & $\mathrm{D}_{25^{\circ} \mathrm{C}} / 10^{-6} \mathrm{~cm}^{2} / \mathrm{s}$ & $\mathrm{R}_{\mathrm{h}} / \AA$ \\
\hline Oregon Green 488 & 368 & $4.11 \pm 0.06$ & $5.95 \pm 0.10$ \\
Rhodamine 6G & 443 & $4.14 \pm 0.05$ & $5.89 \pm 0.09$ \\
Atto655-COOH & 528 & $4.30 \pm 0.15$ & $5.68 \pm 0.19$ \\
Atto655 NHS ester & 625 & $4.14 \pm 0.10$ & $5.86 \pm 0.14$ \\
Atto655 maleimide & 650 & $4.07 \pm 0.10$ & $6.01 \pm 0.11$ \\
Cy5 & 780 & $3.68 \pm 0.09$ & $6.64 \pm 0.22$ \\
Alexa Fluor ${ }^{\circledR}$ 647 & 1130 & $3.25 \pm 0.10$ & $7.52 \pm 0.23$ \\
$\quad+$ MW of the ionic form of the dyes. &
\end{tabular}

Table A.2: Hydrodynamic radii for the globular proteins

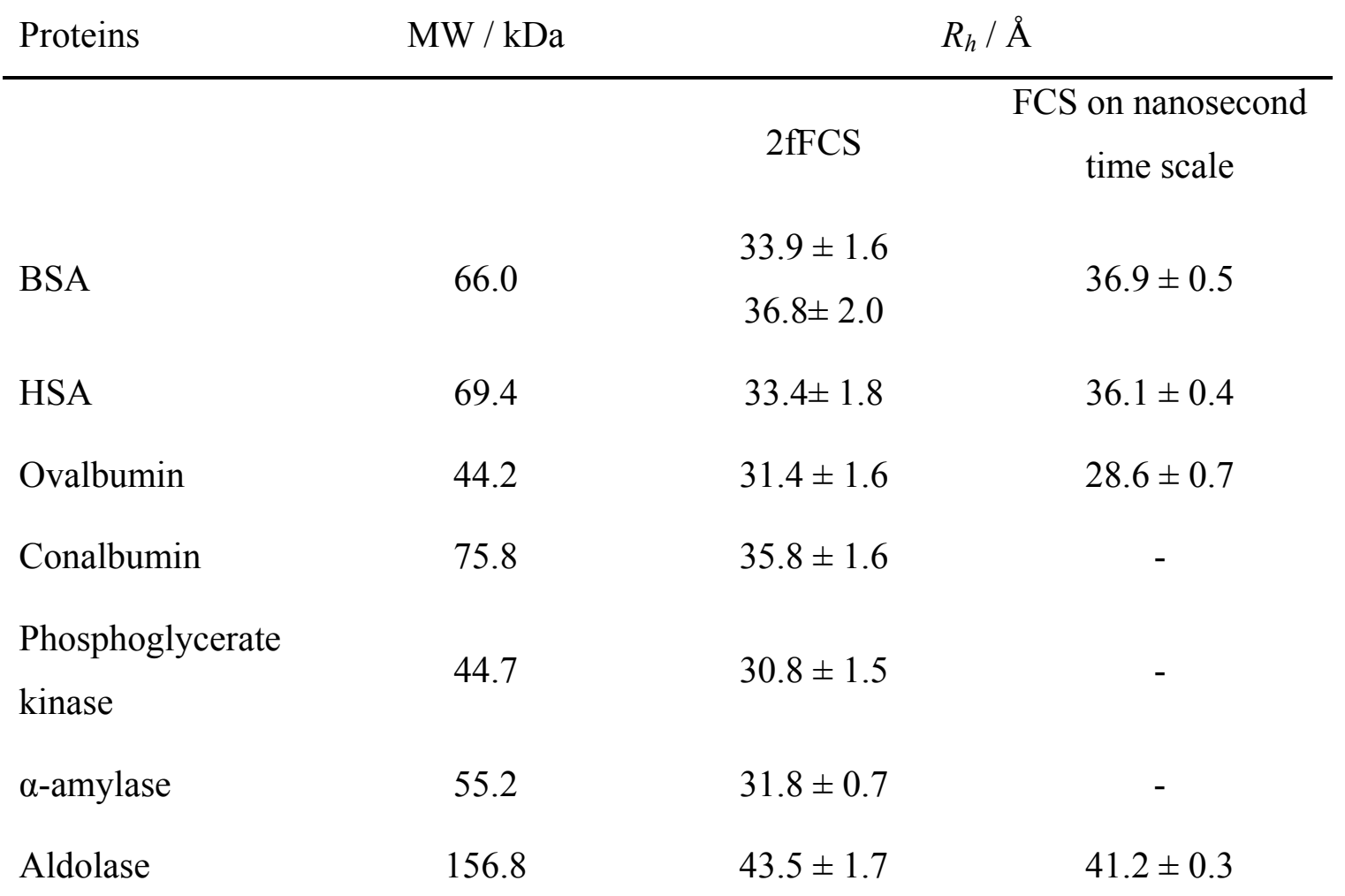




\section{References}

Abramowitz,M. \& Stegun,I.A. (1965) Handbook of Mathematical Functions. Dover, New York.

Altieri,A.S, Hinton,D.P., Byrd,A.R. (1995) Association of Biomolecular Systems via Pulsed Field Gradient NMR Self-Diffusion Measurements. JACS, 117, 7566-7561.

Ambrose,W.P., Goodwin,P.M., Enderlein,J., Semin,D.J., Martin,J.C., Keller,R.A. (1997) Fluorescence photon antibunching from single molecules on a surface. Chem. Phys. Lett., 269, 365-370.

Aragón,S.R. \& Pecora,R. (1975) Fluorescence correlation spectroscopy and Brownian rotational diffusion. Biopolymers, 14, 119-138.

Bahlmann,K. \& Hell,S.W. (2000) Electric field depolarization in high aperture focusing with emphasis on annular apertures. J. Microscopy, 200, 59-67.

Bahlmann,K. \& Hell,S.W. (2000) Depolarization by high aperture focusing. Appl. Phys. Lett., 77, 612-614.

Benda,A., Benes,M., Marecek,V., Lhotsky,A., Hermens,W.Th., Hof,M. (2003) How to determine diffusion coefficients in planar phospholipid systems by confocal fluorescence spectroscopy. Langmuir, 19, 4120-4126.

Benda,A.; Fagulova,V.; Deyneka,A.; Enderlein,J.; Hof,M. (2006) Fluorescence Lifetime Correlation Spectroscopy Combined with Lifetime Tuning: New Perspectives in Supported Phospholipid Bilayer Research. Langmuir, 22, 9580-9585.

Berg,H.C. (1983) Random walks in biology. Princeton, New Jersey.

Berland,K. \& Shen,G. (2003) Excitation Saturation in Two-Photon Fluorescence Correlation Spectroscopy. Appl. Opt., 42, 5566-5576.

Berne,B.J. \& Pecora,R. (2000) Dynamic Light Scattering. Dover, Mineola/New York.

Bier,M. (2008) Accounting for the energies and entropies of kinesin's catalytic cycle. Eur. Phys. J. B, 65, 415-418.

Borsch,M., Turina,P., Eggeling,C., Fries,J.R., Seidel,C.A., Labahn,A., Graber,P. (1998) Conformational changes of the $\mathrm{H}^{+}$-ATPase from Escherichia coli upon nucleotide binding detected by single molecule fluorescence. FEBS Lett , 437, 251-254.

Brown,P.H. \& Schuck,P. (2006) Macromolecular size -and-shape distributions by sedimentation velocity analytical ultracentrifugation. Biophysical J., 90, 4651-4661

Callaghan,P.T. (1991) Principles of Nuclear Magnetic Resonance Microscopy. Clarendon Press, Oxford.

Callaghan,P.T., Codd,S.L., Seymour,J.D. (1999) Spatial coherence phenomena arising from translational spin motion in gradient spin echo experiments. Concepts in Magnetic Resonance, 11, 181-202.

Cole,J. L. \& Hansen,J. C. (1999) Analytical Ultracentrifugation as a 
Contemporary Biomolecular Research Tool. J. Biomol. Tech., 10, 163 -176.

Cordes,Th., Vogelsang,J., Tinnefeld,Ph. (2009) On the mechanism of Trolox as antiblinking and antibleaching reagent. JACS, 131, 5018-5019.

Creighton,T.E. (Ed) (1998) Protein structure. A practical approach, Oxford University Press, New York.

Creighton,T.E. (1999) Encyclopedia of molecular biology. A Wiley Inter-science Publication.

Culbertson,C.T., Jacobson,S.C., Ramsey,J.M. (2002) Diffusion coefficient measurements in microfluidic devices. Talanta, 56, 365-373.

Damaschun,G., Damaschun,H., Gast,K., Misselwitz,R., Miiller,J.J., Pfeil,W., Zirwer,D. (1993) Cold Denaturation-Induced Conformational Changes in Phosphoglycerate Kinase from Yeast. Biochemistry, 32, 7739-7746.

Daniel,R., Caminade,E., Martel,A., Le Goffic,F., Canosa,D., Carrascal,M., Abian,J. (1997) Mass Spectrometric Determination of the Cleavage Sites in Escherichia coli Dihydroorotase Induced by a Cysteine-specific Reagent. J. Biological Chemistry, 272, 26934-26939.

Danielsson,J., Jarvet,J., Damberg,P., Gräslund,A. (2002) Translational diffusion measured by PFG-NMR on full length and fragments of the Alzheimer $A \beta(1-40)$ peptide. Determination of hydrodynamic radii of random coil peptides of varying length. Magn. Reson. Chem., 40, S89-S97.

Davis,S.K. \& Bardeen,C.J (2002) Using two-photon standing waves and patterned photobleaching to measure diffusion from nanometers to microns in biological systems, Rev. Sci. Instrum., 73, 2128-2135.

Debye,P. (1929) Polar Molecules. The Chemical Catalogue Company, New York, pp.77-108.

Dertinger,T.; von der Hocht,I., Benda,A.; Hof,M.; Enderlein,J. (2006) Surface Sticking and Lateral Diffusion of Lipids in Supported Bilayers. Langmuir, 22, 93399344.

Dertinger,T.; Pacheco,V., von der Hocht,I., Hartmann,R., Gregor,I., Enderlein,J. (2007) Two-focus Fluorescence Correlation Spectroscopy: a new tool for accurate and absolute diffusion measurements. ChemPhysChem, 8, $433-443$.

Dertinger,T., Loman,A., Ewers,B., Müller,C.B., Krämer,B., Enderlein,J. (2008) The optics and performance of dual-focus fluorescence correlation spectroscopy. Opt. Express, 16, 14353-14368.

Diez,M., Borsch,M., Zimmermann,B., Turina,P., Dunn,S.D., Graber,P. (2004) Binding of the b-subunit in the ATP synthase from Escherichia coli. Biochemistry, 43, 1054-1064.

Doose,S., Neuweiler,H., Sauer,M. (2005) A Close Look at Fluorescence Quenching of Organic Dyes by Tryptophan. ChemPhysChem, 6, 2277 - 2285. 
Donnert,G., Eggeling,C., Hell,S.,W. (2007) Major signal increase in fluorescence microscopy through darkstate relaxation. Nature Meth., 4, 81-86.

Dunn,B.M. (2001). Overview of pepsin-like aspartic peptidases. Curr Protoc Protein Sci, Unit 21.3.

Ebbinghaus,S., Dhar,A., McDonald,J.D., Gruebele,M. (2010) Protein folding stability and dynamics imaged in a living cell. Nature meth. (published online)

Eggeling,Ch., Widengren,J., Brand,L., Schaffer,J., Felekyan,S., Seidel, C.A.M. (2006) Analysis of Photobleaching in Single-Molecule Multicolor Excitation and Förster Resonance Energy Transfer Measurements. J. Phys. Chem. A, 110, 2979-2995.

Eggeling,Ch., Kask,P., Winkler,D., Jäger,S. (2005) Rapid Analysis of Förster Resonance Energy Transfer by Two-Color Global Fluorescence Correlation Spectroscopy: Trypsin Proteinase Reaction. Biophysical J., 89, 605-618.

Ehrenberg,M. \& Rigler,R., (1974) Rotational Brownian motion and fluorescence intensity fluctuations. Chem. Phys., 64, 390-401.

Einstein,A. (1985) Investigations on the Theory of the Brownian Movement. Dover, New York.

Enderlein,J., Robbins,D.L., Ambrose,W.P., Keller,R.A. (1998) Molecular Shot Noise, Burst Size Distribution, and Single-Molecule Detection in Fluid. J. Phys. Chem. A, 102, 6089-6094.

Enderlein,J \& Sauer,M. (2001) Optimal Algorithm for Single-Molecule Identification with Time-Correlated Single-Photon Counting. J. Phys. Chem. A, 105, 4853.

Enderlein,J., Gregor,I., Patra,D. \& Fitter,J. (2004) Art and artefacts of fluorescence correlation spectroscopy. Curr.Pharm.Biotechnol., 5, 155-161.

Enderlein,J.; Gregor,I.; Patra,D.; Dertinger,T.; Kaupp,B. (2005) Performance of fluorescence correlation spectroscopy for measuring diffusion and concentration. ChemPhysChem, 6, 2324-2336.

Enderlein,J. (2005) Dependence of the optical saturation of fluorescence on rotational diffusion. Chem. Phys. Lett., 410, 452-456.

Enderlein,J. \& Gregor,I. (2005) Using fluorescence lifetime for discriminating detector afterpulsing in fluorescence-correlation spectroscopy. Rev. Sci. Instrum., 76, 033102-033105.

Fasman,G.D. (Ed.) (1989) CRC Practical Handbook of Biochemistry and Molecular Biology, ed., CRC Press.

Fersht,A. (1999) Structure and mechanism in protein science: a guide to enzyme catalysis and protein folding. W.H. Freeman and Company. New York.

Felekyan,S., Kühnemuth,R., Kudryavtsev,V., Sandhagen,C., Becker,W., Seidel,C.A.M. (2005) Full correlation from picoseconds to seconds by time-resolved and time-correlated single photon detection. Rev. Sci. Instrum., 76, 083104. 
Ferrer,M.L., Duchowicz,R.,. Carrasco,B, de la Torre,J.G.,Acuña,A.U., (2001) "The Conformation of Serum Albumin in Solution: A Combined Phosphorescence Depolarization-Hydrodynamic Modeling Study", Biophysical J., 2001, 80, 2422-2430.

Feynman,R. (1964) Feynman's lectures on physics. Addison-Wesley, Reading, MA, USA, chapter 18.4 .

Flecha,F.L.G. \& Levi,V. (2003) Determination of the Molecular Size of BSA by Fluorescence Anisotropy. Biochem. Mol. Biol. Educ., 31, 319-322.

Fischer,H., Polikarpov,I., Craievich,A.F. (2004) Average protein density is a molecular-weight-dependent function. Protein Science , 13, 2825-2828.

Fitter,J. \& Haber-Pohlmeier,S. (2004) Structural Stability and Unfolding Properties of Thermostable Bacterial $\alpha$-Amylases: A Comparative Study of Homologous Enzymes. Biochemistry, 43, 9589-9599.

Gekko,K. \& Noguchi,H. (1979) Compressibility of globular proteins in water at 25 degrees C. J. Phys. Chem., 83, 2706-2714.

Gregor,I., Patra,D., Enderlein,J. (2005) Optical Saturation in Fluorescence Correlation Spectroscopy under Continuous-Wave and Pulsed Excitation. ChemPhysChem, 6,164 170.

Gregor,I. \& Enderlein,J. (2007) Time-resolved methods in biophysics. 3. Fluorescence lifetime correlation spectroscopy. Photochem. Photobiol. Sci., 6, 13-18.

Hamadani,K.M. \& Weiss,S. (2008) Nonequilibrium Single Molecule Protein Folding in a Coaxial Mixer. Biophysical J., 95, 352-365

Harding,S.E. (1997) The intrinsic viscosity of biological macromolecules. Progress in measurement, interpretation and application to structure in dilute solution. Prog. Biophys. molec. Biol., 68, 207-262.

Heilemann,M., Margeat,E., Kasper,R., Sauer,M., Tinnefeld,Ph. (2005) Carbocyanine Dyes as Efficient Reversible Single-Molecule Optical Switch. JACS, 127, 3801-3806.

Hess,S.T. \& Webb,W.W. (2002) Focal volume optics and experimental artifacts in confocal fluorescence correlation spectroscopy. Biophysical J., 83, 2300-2317.

Hinterdorfer,P. \& van Qijen,A. (Eds.) (2009) Handbook of Single-Molecule Biophysics, Springer. New York

Humpoličková,J., Gielen,E., Benda,A., Fagulova,V., Vercammen,J., VandeVen,M., Hof,M., Ameloot,M., Engelborghs,Y. (2006) Probing Diffusion Laws within Cellular Membranes by Z-Scan Fluorescence Correlation Spectroscopy. Biophysical J. Biophys. Lett., 91, L23-L25.

Humpolíčková,J.; Benda,A.; Sýkora,J.; Macháň,R.; Kral,T.; Gasinska,B.; Enderlein,J.; Hof,M. (2008) Equilibrium Dynamics of Spermine-Induced Plasmid DNA Condensation Revealed by Fluorescence Lifetime Correlation Spectroscopy. Biophysical J., 94, L17-19. 
Huang,B.X., Kim,H.-Y.; Dass,C. (2004) Probing three-dimensional structure of bovine serum albumin by chemical cross-linking and mass spectrometry. Journal of the American Society for Mass Spectrometry, 15, 1237-1247.

Ishikawa,E., Hashida,S., Kato,Y., ImuraH. (1987) Sensitive Enzyme lmmunoassay of Human Growth Hormone for Clinical Application: A Review. J Clin. Lab Anal., 1, 238-242.

Jaffiol,R., Blancquaert,Y., Delon,A., Derouard,J. (2006) Spatial fluorescence cross-correlation spectroscopy. Appl.Opt., 45, 1225-1235.

Kapusta,P.; Wahl,M.; Benda,A.; Hof,M.; Enderlein,J. (2007) Fluorescence Lifetime Correlation Spectroscopy. J. Fluoresc., 17, 43-48.

Kask,P., Piksarv,P., Mets,Ü., Pooga,M., Lippmaa,E. (1987) "Fluorescence correlation spectroscopy in the nanosecond time range: rotational diffusion of bovine carbonic anhydrase B", Eur. Biophys. J., 14, 257-261.

Kask,P., Piksarv,P., Mets,Ü. (1985) Fluorescence correlation spectroscopy in the nanosecond time range: Photon antibunching in dye fluorescence. Eur. Biophys. J., 12, 163166.

Kiefhaber,T., Rudolph,R., Kohler,H.H. \& Buchner,J. (1991) Protein aggregation in vitro and in vivo: a quantitative model of the kinetic competition between folding and aggregation. Biotechnology (N.Y.), 9, 825-829.

Klar,Th.A., Jakobs,S. Dyba,M., Egner,A., Hell,S.W. (2000) Fluorescence microscopy with diffraction resolution barrier broken by stimulated emission. PNAS, $\mathbf{9 7}$, 8206-8210.

Klar,Th.A., Egner,A., Hell,S.W. (2001) Breaking Abbe's diffraction resolution limit in fluorescence microscopy with stimulated emission depletion beams of various shapes. Phys. Rev. E, 64, 066613-1-066613-9.

Koenig,S.H. (1975) Brownian Motion of an Ellipsoid. A Correction to Perrin's Results. Biopolymers, 14, 2421-2423.

Köhn,F., Hofkens,J., Gronheid,R., Van der Auweraer,M., De Schryver,F.,C. (2002) Parameters Influencing the On- and Off-Times in the Fluorescence Intensity Traces of Single Cyanine Dye Molecules. J. Phys.Chem. A, 106, 4808-4814.

Knight,A. (Ed.) (2009) Single Molecule Biology. Elsevier Inc., New York.

Lakowicz,J.R. (1999) Principles of Fluorescence Spectroscopy, Kluwer Academic/Plenum Publishers, New York, 1999, pp. 291-320.

Lacowicz,J.R. (2006) Principles of Fluorescence Spectroscopy. Springer. New York.

Liu,W., Cellmer,T., Keerl,D., Prausnitz,J.M. \& Blanch,H.W. (2005) Interactions of lysozyme in guanidinium chloride solutions from static and dynamic light-scattering measurements. Biotechnol.Bioeng., 90, 482-490.

Loman,A., Dertinger,T., Koberling,F., Enderlein,J. (2008) Comparison of optical 
saturation effects in conventional and dual-focus fluorescence correlation spectroscopy. Chem. Phys. Lett., 459, 18-21.

Loman A., Gregor I., Stutz Ch., Mund M., Enderlein J. (2010) Measuring rotational diffusion of macromolecules by fluorescence correlation spectroscopy. Photochem. Photobiol. Sci., in press

Lu,M.-H., Rosenblatt,Ch., Yager,P. (1993) Influence of $\mathrm{pH}$ on the precursors of phospholipid tubules in methanolic solution, Chemistry and Physics of Lipids, 65, 77-84

Magde,D., Elson,E., Webb,W.W. (1972) Thermodynamic fluctuations inreacting systm - measurement by fluorescence correlation spectroscopy. Phys. Ref. Lett., 29, 705-708.

Magde,D., Elson,E., Webb,W.W. (1974) Fluorescence Correlation Spectroscopy. 11. An Experimental Realization. Biopolymers, 13, 29

Magde,D., Webb,W.W., Elson,E. (1978) Fluorescence correlation spectroscopy. III. Uniform translation and laminar flow. Biopolymers, 17, 361-376. York.

Mark,J.E (Ed) (2007) Physical Properties of Polymers Handbook. Springer. New

Meissner,O., Häberlein,H. (2003). Lateral mobility and specific binding to GABAA receptors on hippocampal neurons monitored by fluorescence correlation spectroscopy. Biochemistry, 42, 1667-1672.

Moerner,W.E. \& Kador,L. (1989) Optical detection and spectroscopy of single molecules in a solid. Phys. Rev. Lett., 62, 2535-2538.

Morse,P.M. \& Feshbach,H. (1953) Methods of Theoretical Physics. McGraw-Hill, New York, chapter 7.

Murphy,R.M. \& Tsai,A.M. (Eds.) (2006) Misbehaving Proteins Protein (Mis)Folding, Aggregation, and Stability. Springer. New York.

Murtaza,Z., Herman,P., Lakowicz,J.R. (1999) Synthesis and spectral characterization of a long-lifetime osmium (II) metal-ligand complex: a conjugatable red dye for applications in biophysics. Biophys. Chem., 80, 143-151.

Müller,C.B., Weiß,K., Richtering,W., Loman,A., Enderlein.J (2008a) Calibrating Differential Interference Contrast Microscopy with dual-focus Fluorescence Correlation Spectroscopy. Opt. Express, 16, 4322-4329.

Müller,C.B., Loman,A., Richtering,W., Enderlein.J (2008b) Dual-Focus Fluorescence Correlation Spectroscopy of Colloidal Solutions: Influence of Particle Size, $J$. Phys. Chem. B, 112, 8236-8240.

Müller,C.B., Loman,A., Pacheco,V., Koberling,F., Willbold,D., Richtering,W., Enderlein.J (2008c) Precise measurement of diffusion by multi-color dual-focus fluorescence correlation spectroscopy. EPL, 83, 46001p1 - 46001p5.

Müller,B.K., Zaychikov,E., Bräuchle,Ch., Lamb,D.C. (2005) Pulsed Interleaved Excitation. Biophysical J., 89, 3508-3522. 
Nagy,A., Wu,J., Berland,K.M. (2005) Observation volumes and \{gamma\}-factors in two-photon fluorescence fluctuation spectroscopy. Biophysical J., 89, 2077-2090.

Nguyen,D.C.; Keller,R.A.; Jett,J.H.; Martin,J.C. (1987) Detection of single molecules of phycoerythrin in hydrodynamically focused flows by laser-induced fluorescence, Anal. Chem., 59, 2158-2161.

Nishimura,G. \& Kinjo,M. (2004) Systematic Error in Fluorescence Correlation Measurements Identified by a Simple Saturation Model of Fluorescence. Anal. Chem., 76, 1963-1970.

Nomarski,G. (1970) Interference Microscopy - State of Art and Its Future. J. Opt. Soc. Am., 60, 1575-1575.

Orrit,M. \& Bernard,J. (1990) Single pentancene molecules detected by fluorescence excitation in a p-Terphenyl crystal. Phys. Rev. Lett., 65, 2716-2719.

Otosu,T., Nishimoto,E., Yamashita,S. (2010) Multiple conformational state of human serum albumin around single tryptophan residue at various $\mathrm{pH}$ revealed by timeresolved fluorescence spectroscopy. Journal of Biochemistry, 147, 191-200.

Parmar,A.S. \& Muschol,M. (2009) Hydration and Hydrodynamic Interactions of Lysozyme: Effects of Chaotropic versus Kosmotropic Ions, Biophysical J., 97, 590-598.

Pei,H, Germann,M.W., Allison, S.A. (2009) Translational Diffusion Constants of Short Peptides: Measurement by NMR and Their Use in Structural Studies of Peptides. J. Phys. Chem. B, 113, 9326-9329.

Perrin,F. (1934) Mouvement brownien d'un ellipsoide (I). Dispersion dielectrique pour des molecules ellipsoidales. J. Phys. Radium Ser. VII, 5, 497-511.

Perrin,F. (1936) "Mouvement brownien d'un ellipsoide (I). Dispersion dielectrique pour des molecules ellipsoidales", J. Phys. Radium Ser. VII, 7, 1-11.

Perroud,T.D., Huang,B., Wallace,M.I., Zare,R.N. (2003) Photon counting histogram for one-photon excitation. ChemPhysChem., 4, 1121-1123.

Perroud,T.D., Huang,B. \& Zare,R.N. (2005) Effect of bin time on the photon counting histogram for one photon excitation. ChemPhysChem., 6, 905-912.

Petrasek,Z. \& Schwille,P. (2008) Precise measurement of diffusion coefficients using scanning fluorescence correlation spectroscopy. Biophysical J., 94, 1437-1448.

Ries,J. \& Schwille,P. (2006) Studying Slow Membrane Dynamics with Continuous Wave Scanning Fluorescence Correlation Spectroscopy. Biophysical J., 91, 1915-1924.

Rigler,R. \& Widengren,J. (1990) Bioscience, 3, 180-183.

Rigler,R., Mets,U., Widengren,J., Kask,P. (1993) Fluorescence correlation spectroscopy with high count rate and low background: analysis of translational diffusion. Eur. Biophys. J., 22, 169-175.

Rigler,R. \& Elson,E. (Eds.) (2001) Fluorescence Correlation Spectroscopy. Springer. New York. 
Rigneault,H. \& Lenne,P.-F. (2003) Fluorescence correlation spectroscopy on a mirror, J. Opt. Soc. Am. B, 20, 2203-2214. New York.

Rubinshtein,M. \& Colby,R.H. (2003) Polymer physics. Oxford University Press,

Sabaté,R., Estelrich,J. (2001) Aggregation Characteristics of Ovalbumin in $\beta$-Sheet Conformation Determined by Spectroscopy. Biopolymers, 67, 113-120.

Scwille,P. \& Haustein,E. (2001) Fluorescence Correlation Spectroscopy: an introduction to its concepts and applications. Online published: http://www.biophysics.org/Portals/1/PDFs/Education/schwille.pdf.

Selvin,P.R. \& Ha,T. (Eds.) (2008) Single-Molecule Techniques: A Laboratory Manual. Cold Spring Harbor. New York.

Sherman,E., Itkin,A., Kuttner,J.Y., Rhoades,E., Amir,D., Haas,A., Haran,G. (2008) Using Fluorescence Correlation Spectroscopy to Study Conformational Changes in Denatured Proteins. Biophysical J., 94, 4819-4827.

Spragg,P. S., Wilcox,J.K., Roche,J.J., Barnett,W.A. (1976) The Association of Yeast Phosphoglycerate Kinase (EC 2.7.2.3). Biochemistry J., 153, 423-428

Squire,P.G. \& Himmel,M.E. (1979) Hydrodynamics and protein hydration. Arch. Biochem. Biophys., 196, 165-177.

Sun,S.F. (Ed.) (2004) Physical chemistry of macromolecules: basic principles and issues. J.Wiley \& Sons, Inc. Hoboken. New Jersey.

Tabak,M., de Sousa Neto,D., Salmon,C.E.G. (2006) On the Interaction of Bovine Serum Albumin (BSA) with Cethyltrimethyl Ammonium Chloride Surfactant: Electron Paramagnetic Resonance (EPR) Study. Brazilian J. Phys., 36, 83-89.

Tai,H.C. (2004) X-ray crystallographic studies of bovine serum albumin and helicobacter pylori thioredoxin-2. thesis, Department of Chemistry, University of Saskatchewan, Canada.

Thompson,W.J. (1994) Angular momentum. John Wiley \& Sons, New York, NY, USA.

de la Torre;G.J., Carrasco,B., Harding,S.E. (2000) Calculation of hydrodynamic properties of globular proteins from their atomic level structure. Biophysical J., 78, 719-30.

Tsay,J.M., Dose,S., Weiss,S. (2006) Rotational and Translational Diffusion of Peptide-Coated CdSe/CdS/ZnS Nanorods Studied by Fluorescence Correlation Spectroscopy. JACS, 128, 1639-1647.

Vestling,M.M., Kelly,M.A., Fenselau,C. (1994) Optimization by mass spectrometry of a tryptophan-specific protein cleavage reaction. Rapid Commun. Mass Spectrom., 8, 786790.

Vogelsang,J., Kasper,R., Steinhauer,Ch., Person,B., Heilemann,M., Sauer,M., Tinnefeld,Ph. (2008) A reducing and oxidizing system minimizes photobleaching and blinking of fluorescent dyes. Angew. Chem. Int. Ed., 47, 5465-5469. 
Wahl,M., Gregor,I., Patting,M., Enderlein,J. (2003) Fast calculation of fluorescence correlation data with asynchronous time-correlated single-photon counting. Opt. Express, 11, 3583-3591.

Weljie,A.M., Yamniuk,A.P., Yoshino,H., Izumi,Y. \& Vogel,H.J. (2003) Protein conformational changes studied by diffusion NMR spectroscopy: Application to helixloop-helix calcium binding proteins. Protein Science, 12, 228-236.

Widengren,J., Rigler,R., Mets,U.J. (1994) Triplet-State Monitoring by Fluorescence Correlation Spectroscopy. J. Fluorescence, 4, 255-258.

Widengren,J. \& Schwille,P. (2000) Characterization of photoinduced isomerization and back-isomerization of the cyanine dye Cy5 by Fluorescence Correlation Spectroscopy. J. Phys. Chem. A., 104, 6416-6428.

Widengren,J.,Schweinberger,E., Berger,S., Seidel,C.A.M. (2001) Two New Concepts to Measure Fluorescence Resonance Energy Transfer via Fluorescence Correlation Spectroscopy: Theory and Experimental Realizations. J. Phys. Chem. A, 105, 6851-6866.

Wilson,K. \& Walker,J. (Eds.) (2010) Principles and Techniques of Biochemistry and Molecular Biology. Cambridge University Press. New York.

Wishnia,A., Weber,I., Warner,R. (1961) The Hydrogen-ion Equilibria of Conalbumin. JACS, 83, 2031-2218.

Woffbeis,J (Ed) (1992) Fluorescence Spectroscopy. Springer. Berlin.

Yamniuk,A.P., Nguyen,L.T., Hoang,T.T., Voge,1H.J. (2004) Metal Ion Binding Properties and Conformational States of Calcium- and Integrin-Binding Protein, Biochemistry, 43, 2558-2568.

Zander,Z., Enderlein,J., Keller,R.A. (Eds.) (2002) Single-molecule detection in solution methods and applications. VCH-Wiley. Berlin/New York.

Zwillinger,D. (1990) Handbook of differential equations. Academic Press, San Diego, CA, USA, chapter 96. 



\section{Acknowledgments}

It is my great pleasure to thank many people whom I met during my $\mathrm{PhD}$ time. The present work would have never been completed without their help and support.

First of all I would like to thank my supervisor, Jörg Enderlein, for giving me an opportunity to work with you. Jörg, thank you for encouraging, supervising and supporting me from my first day in the group to the final stage of this work. Thank you for finding time for discussions when I needed it. I am grateful for all ideas, knowledge, and experience you shared with me. Besides new knowledge and skills, I learned from you that research is great fun. I appreciate that you were not only my teacher but also a colleague. I admit I have enjoyed my $\mathrm{PhD}$ time very much. Although having to follow you around Germany a few times was somewhat challenging, it was a great opportunity to explore the country.

Iris von der Hocht, you were the person who helped to record my first experimental data. Thanks for your patience while teaching me how to use the set-up and label proteins.

Ingo Gregor, I had missed the opportunity to discuss with you all questions, doubts, and ideas I had in Tübingen. Ingo, thanks a lot for sharing your experience and routines. I am very glad that you rejoined the group.

Jana Humpolíčková and Tyler Arbour, you were my valued colleagues in Tübingen. Jana, I was glad you were there in the office to share my frustrating moments and discuss my working problems. We had been laughing a lot, which was very helpful and alleviated most troubles. Tyler, you were so enthusiastic about science that you could not help but infect me with your passion. Since then I discovered that reading Nature and Science can be just as fun as reading entertainment magazines.

I would like to thank all people from our group, Mira Prior, Phillip Kroehn, Christoph Pieper, Qui Van, Stefan Lakämper and Bernd Müller. All of you create a nice and inspiring working environment. It made me come to the office much earlier than I otherwise would. Sorry guys I still did not make it in time for joining you for lunch every day. Steffi Reiter, your joy and energy were contagious, thanks for that.

I would like to thank Antje Erdmann and the coordination team of GGNB program for their help with all organization questions and paperwork.

I am very thankful to Elke Zech for being such a friendly and caring person, and for always being ready to help me out with administrative issues and bureaucratic problems.

Iwan Schaarp, Schanila Nawaz, and Frédéric Eghiaian, it was great to have you around. The feeling that I was not the only person who worked late was very supporting. Besides, thanks a lot for matching your lunch timing with mine. Fred, your proofreading was very helpful. Sergei Zhukovsky, thanks for your support and being non-expert reader.

Last but not least, warmest thanks go to my family: папа, мама и Миша, мой младший братишка, спасибо вам за поддержку в сложные моменты и веру в мои силы. 



\section{Curriculum Vitae}

NAME: Anastasia Loman

BIRTH: March 22, 1980 in Minsk, Belarus

\section{EDUCATION}

June 1997 Secondary school No.166, Minsk Belarus.

- Graduated with silver medal (Educational Ministry Award)

June 2002 Belarusian State University, Minsk, Belarus.

- Diploma in Physics.

Subject: Special properties of oxygen quenching of singlet and triplet states watersoluble porphyrin Graduated with honors.

Nov 2006 - March 2010 PhD student in the group of Single molecule spectroscopy and imaging for Biophysics and Complex systems of Prof. Dr. J. Enderlein, Germany.

\section{EMPLOYMENT}

Aug 2002 - Oct 2003 research assistant at Institute of Molecular and Atomic Physics of National Academy of Sciences of Belarus

Nov 2003 - Aug 2005 research assistant at Institute of Biophysics and Cellular Engineering of

National Academy of Sciences of Belarus

Sep 2005 - Oct 2006 research assistant at Leiden University, the Netherlands 



\section{Publications}

- $\quad$ Müller C. B., Weiß K., Richtering W., Loman A., Enderlein J. (2008) Calibrating Differential Interference Contrast Microscopy with dual-focus Fluorescence Correlation Spectroscopy. Opt. Expr. 16, $4322-4329(*)$.

- Loman A., Dertinger T., Koberling F., Enderlein J. (2008) Comparison of optical saturation effects in conventional and dual-focus fluorescence correlation spectroscopy. Chem. Phys. Lett. 459, 18-21 (*).

- $\quad$ Müller C.B., Loman A., Richtering W., Enderlein J. (2008) Dual-Focus Fluorescence Correlation Spectroscopy in Colloidal Solution: Influence of Particle Size. J. Phys. Chem. B 112, 8236-8240.

- Müller C.B., Loman A., Pacheco V., Koberling F., Willbold D., Richtering W., Enderlein J. (2008) Precise Measurement of Diffusion by Multi-Color Dual-Focus Fluorescence Correlation Spectroscopy. Eur. Phys. Lett., 83, $46001\left(^{*}\right)$.

- $\quad$ Dertinger T., Loman A., Ewers B., Müller C.B., Krämer B., Enderlein J. (2008) The optics and performance of dual-focus fluorescence correlation spectroscopy. Opt. Expr. 16, $14353-14368(*)$.

- $\quad$ Müller C.B., Eckert T, Loman A., Enderlein J, Richtering W. (2009) Dual-focus fluorescence correlation spectroscopy: a robust tool for studying molecular crowding. Soft Matter, 5, 1358-1366.

- $\quad$ Müller C.B., Weiß K., Loman A. Enderlein J., Richtering, W. (2009) Remote temperature measurements in femto-liter volumes using dual-focus-Fluorescence Correlation Spectroscopy. Lab on a Chip, 9, 1248-1253.

- $\quad$ Loman A., Gregor I., Stutz Ch., Mund M., Enderlein J. (2010) Measuring rotational diffusion of macromolecules by fluorescence correlation spectroscopy. Photochemical \& Photobiological Sciences, in press (*).

$\left(^{*}\right)$ the results presented in these publications are collected in the present thesis. 